\title{
Clinical features and prognostic factors of listeriosis: the MONALISA national prospective cohort study
}

\author{
Caroline Charlier MD ${ }^{1,2,3,4}$, Élodie Perrodeau $\mathrm{MSc}^{5}$, Alexandre Leclercq MSc ${ }^{1,2,3}$, Benoît Cazenave MD \\ Benoît Pilmis MD ${ }^{1,4}$, Benoît Henry MD ${ }^{4,6}$, Amanda Lopes $\mathrm{MD}^{7}$, Mylène M. Maury PhD ${ }^{1,2,3}$, \\ Alexandra Moura $\mathrm{PhD}^{2,3}$, François Goffinet $\mathrm{MD}^{8}$, Hélène Bracq Dieye ${ }^{1,2,3}$, Pierre Thouvenot ${ }^{1,2,3}$, \\ Marie-Noëlle Ungeheuer MD ${ }^{9}$, Mathieu Tourdjman $\mathrm{MD}^{10}$, Véronique Goulet MD ${ }^{10}$, Henriette de Valk $\mathrm{MD}^{10}$, \\ Prof. Olivier Lortholary $\mathrm{MD}^{4}$, Prof. Philippe Ravaud MD ${ }^{5,11}$ and Prof. Marc Lecuit MD $\mathrm{MD}^{1,2,3,4}$ \\ on behalf of the MONALISA study group
}

\footnotetext{
${ }^{1}$ Institut Pasteur, French National Reference Center and WHO Collaborating Center for Listeria, Paris, France

${ }^{2}$ Institut Pasteur, Biology of Infection Unit, Paris, France

${ }^{3}$ Inserm U1117, Paris, France

${ }^{4}$ Paris Descartes University, Sorbonne Paris Cité, Necker-Pasteur Infectiology Centre, Necker-Enfants Malades University Hospital, Institut Imagine, Assistance Publique-Hôpitaux de Paris, Paris, France

${ }^{5}$ Centre of Research in Epidemiology and Statistics Sorbonne Paris Cité, METHODS Team, UMR 1153 , INSERM, Paris Descartes University, Sorbonne Paris Cité, Paris, France

${ }^{6}$ Pierre et Marie Curie University, Pitié Salpétrière University Hospital, Assistance Publique-Hôpitaux de Paris, Paris, France

${ }^{7}$ Denis Diderot University, Department of Internal Medicine, Lariboisière Hospital, Assistance PubliqueHôpitaux de Paris, Paris, France

${ }^{8}$ Paris Descartes University, Sorbonne Paris Cité, Port Royal Maternity, Department of Obstetrics, Cochin Port Royal Hospital, Assistance Publique-Hôpitaux de Paris, Paris, France

${ }^{9}$ Institut Pasteur, Center for Translational Science, ICAReB Biobanking Platform, Paris, France

${ }^{10}$ Infectious Disease Department, The French Public Health Agency, Saint Maurice, France

${ }^{11}$ Columbia University, Mailman School of Public Health, New York, NY, USA
}

Correspondence to Caroline Charlier and Marc Lecuit caroline.charlier@pasteur.fr and marc.lecuit@pasteur.fr

Institut Pasteur

Biology of Infection Unit

French National Reference Center and WHO collaborating Center Listeria

28 rue du Dr. Roux, 75015 Paris, France

Phone: +33140613420

Fax: +33140613421 


\section{RESEARCH IN CONTEXT}

\section{Evidence before this study}

We searched PubMed on June 30, 2016, for English-language cohort studies published since Jan 1, 1980, of patients with invasive listeriosis worldwide with the keywords "listeria", "listeriosis", "maternal", and "neurolisteriosis". Studies had to include epidemiological or clinical data on listeriosis. All clinical forms of infection were included (bacteraemia, neurolisteriosis, and maternal-neonatal infection). Host risk factors for listeriosis have been well identified, but the clinical features and prognostic factors of the disease are based on retrospective studies compiling heterogeneous data or random collected cases. Furthermore, no clinical trial has ever been done and medical management is not evidence based.

\section{Added value of the study}

Our study is the first prospective clinical study focusing on all forms of invasive listeriosis. The study is based on a national mandatory system that allowed the nearly complete capture of microbiologically proven cases. The study shows a higher burden of listeriosis than reported before: more than $80 \%$ of infected mothers experienced major fetal or neonatal

complications (fetal loss, very high prematurity, early or late onset disease); only $39 \%$ of patients with neurolisteriosis survived and fully recovered. The study provides important new data to improve management and predict outcome in listeriosis, such as determination of the time window for fetal losses $(<29$ weeks of gestation and $<3$ days of adequate management) and new factors independently associated with mortality. Our data show the deleterious effect of adjunctive dexamethasone in neurolisteriosis, and argue for the use of betalactam and gentamicin or co-trimoxazole over other antimicrobials for bacteraemia and neurolisteriosis.

\section{Implications of all the available evidence}

Given the practical difficulties in completing clinical trials in listeriosis, these results could guide clinical practice and suggest that combined amoxicillin and gentamicin should be considered the first-line combination in invasive listeriosis, and that adjunctive dexamethasone should be avoided in cases of confirmed listeriosis.

\section{ABSTRACT}

Background: Listeriosis is a severe foodborne infection and a notifiable disease in France. We did a nationwide prospective study to characterise its clinical features and prognostic factors.

Methods: MONALISA was a national prospective observational cohort study. We enrolled eligible cases declared to the National Reference Center for Listeria (all microbiologically proven) between Nov 3, 2009, and July 31, 2013, in the context of mandatory reporting. The outcomes were analysis of clinical features, characterisation of Listeria isolates, and determination of predictors of 3-month mortality or persisting impairment using logistic regression. A hierarchical clustering on principal components was also done for neurological and bacteraemic cases. The study is registered at ClinicalTrials.gov, number NCT01520597.

Findings: We enrolled 818 cases from 372 centres, including 107 maternal-neonatal infections, 427 cases of bacteraemia, and 252 cases of neurolisteriosis. Only five $(5 \%)$ of 107 pregnant women had an uneventful outcome. 26 (24\%) of 107 mothers experienced fetal loss, but never after 29 weeks of gestation or beyond 2 days of admission to hospital. Neurolisteriosis presented as meningoencephalitis in 212 (84\%) of 252 patients; brainstem involvement was only reported in $42(17 \%)$ of 252 patients. 3-month mortality was higher for bacteraemia than neurolisteriosis (hazard ratio [HR] $0 \cdot 54$ [95\% CI $0 \cdot 41-0 \cdot 69], \mathrm{p}<0 \cdot 0001$ ). For both bacteraemia and neurolisteriosis, the strongest mortality predictors were ongoing cancer (odds ratio [OR] $5 \cdot 19$ [95\% CI 3.018.95], $\mathrm{p}<0 \cdot 0001$ ), multi-organ failure (OR 7.98 [4.32-14.72], $\mathrm{p}<0 \cdot 0001$ ), aggravation of any pre-existing organ dysfunction (OR 4.35 [2.79-6.81], $\mathrm{p}<0.0001$ ), and monocytopenia (OR $3 \cdot 70$ [1.82-7.49], $\mathrm{p}=0.0003$ ). Neurolisteriosis mortality was higher in blood-culture positive patients (OR $3 \cdot 67[1 \cdot 60-8 \cdot 40], p=0 \cdot 002)$ or those receiving adjunctive dexamethasone (OR $4 \cdot 58$ [1.50-13.98], $\mathrm{p}=0 \cdot 008)$.

Interpretation: The severity of listeriosis is higher than reported elsewhere. We found evidence of a significantly reduced survival in patients with neurolisteriosis treated with adjunctive dexamethasone, and also determined the time window for fetal losses. MONALISA provides important new data to improve management and predict outcome in listeriosis.

Funding: Programme Hospitalier Recherche Clinique, Institut Pasteur, Inserm, French Public Health Agency. 


\section{INTRODUCTION}

Listeria monocytogenes causes listeriosis, a severe foodborne bacterial infection. L monocytogenes is the foodborne pathogen associated with the highest case-fatality rate in the western hemisphere, where its incidence is estimated at around three to six cases per 1 million population per year. ${ }^{1,2}$ Invasive listeriosis is classified in three forms: bacteraemia, neurolisteriosis, and maternal-neonatal infection; localised forms are also reported.3-5 Epidemiological studies have identified host risk factors for bacteraemia and neurolisteriosis, including older age, innate and cellular immune defects, malignancies, HIV infection, cirrhosis, diabetes mellitus, alcoholism, and immunosuppressive therapies.6 However, all clinical and most epidemiological studies have been retrospective and have compiled data from heterogeneous timeframes and geographical areas..$^{3-10}$ Thus, a precise analysis of disease presentation based on homogeneous and prospectively acquired data is lacking. Furthermore, because listeriosis cases are relatively rare and scattered, no clinical trial has been done, and therapeutic guidelines are not evidence-based. The prognosis for listeriosis has not improved over the past decades. ${ }^{3,7}$

Listeriosis has been a notifiable disease in France since 1999, with mandatory notification of culture-confirmed cases and submission of isolates to the National Reference Center for Listeria (NRCL). A recent capturerecapture study estimated at $85-87 \%$ the exhaustiveness of the national surveillance system for the $2008-13$ period, and since 2000 , an average $98 \%$ of nationally reported cases have isolates submitted to the NRCL.11 Taking advantage of the quasi-exhaustiveness of this surveillance system, we implemented the prospective Multicentric Observational NAtional Study on LISteriosis and ListeriA (MONALISA), a nationwide cohort, to precisely characterise the clinical patterns and identify prognostic factors for invasive listeriosis.

\section{METHODS}

\section{Study design and patients}

MONALISA is a national prospective observational cohort study. We enrolled eligible cases declared to the NRCL (all microbiologically proven), in the context of mandatory reporting. We report the study in accordance with the STROBE reporting guidelines for observational studies. ${ }^{12}$ A case was defined as a patient in whom L monocytogenes was isolated from a normally sterile site. We included all cases from Nov 3, 2009, to July 31, 2013, and classified these as maternal-neonatal infection, bacteraemia, neuro- listeriosis, or other form. Patients originated from 372 centres within France. Maternal-neonatal infection was defined when $L$ monocytogenes was isolated in pregnant women, fetuses, or infants 1 month old or younger. When $L$ monocytogenes was isolated from samples of both mother and her infant, the event was counted as a single case. Bacteraemia was defined when $L$ monocytogenes was isolated from blood culture, without neurolisteriosis or maternal- neonatal infection. Neurolisteriosis was defined when $L$ monocytogenes was isolated from the cerebrospinal fluid (CSF) or a brain abscess, when $L$ monocytogenes was isolated from blood cultures in a patient with otherwise unexplained neurological symptoms (altered consciousness, seizures, nuchal rigidity, or focal neurological symptoms), when L monocytogenes was isolated from blood cultures and identified in CSF by PCR, and finally, in a single patient with meningitis, when $L$ monocytogenes was identified in CSF by PCR and all other microbiological analyses were negative. Patients with neurolisteriosis and positive blood cultures were classified as neurolisteriosis cases. Other forms were defined by the isolation of $L$ monocytogenes from other normally sterile sites. Data on past history, features at admission, and treatments were collected. All patients (or their legal representatives when they were unable to consent) provided written informed consent. In accordance with French legislation, MONALISA received institutional review board approval by the local ethics committee (Comité de Protection des Personnes Ile-de-France 3, Nov 6, 2009). Biospecimen collection was declared to the Ministry of Research (DC 2012-1698 coll 16). In accordance with French legislation, data from patients who died before contact were included, provided that their next of kin agreed.

\section{Procedures}

L monocytogenes isolates were identified with API Listeria (BioMérieux, Marcy l'Etoile, France) and sequenced using Illumina technology (appendix). Multilocus sequence types and inlA gene variants were extracted from genome assemblies using Lm-BIGSdb.13 DNA extraction was done using the DNeasy blood and tissue extraction kit (Qiagen, Aarhus, Denmark), from $5 \mathrm{~mL}$ of liquid cultures grown overnight at $35^{\circ} \mathrm{C}$ in brain-heart infusion medium under aerobic conditions, following the manufacturer's protocol. Library preparation was carried out using the Nextera XT DNA sample kit and whole genome sequencing was done on the NextSeq 500 platform using $2 \times 150 \mathrm{bp}$ runs (Illumina, San Diego, CA, USA) at a minimum coverage of $40 \times$. Paired-end reads were trimmed with fqCleaner v05 to eliminate adapter sequences and discard reads with Phred scores of 20 or less. Assemblies were obtained using CLC Assembly Cell 430 (Qiagen, Aarhus, Denmark).

\section{Outcomes}

We analysed clinical features, characterised $L$ monocytogenes isolates, and determined predictors of 3-month mortality or persisting impairment using logistic regression. 


\section{Statistics}

The sample size was a convenience sample, determined by the number of eligible cases during the study period. We investigated the relation between unfavourable outcome and potential predictors by doing a multivariable logistic analysis. Unfavourable outcome was defined as fetal loss for maternal-neonatal infections, 3-month mortality for bacteraemia and neurolisteriosis, and also persisting neurological impairment for neurolisteriosis. Variables showing associations at a significance level of $\alpha=0 \cdot 20$ in a univariable analysis were selected for inclusion in the multivariable model and a stepwise selection was done. Missing values were imputed using a multivariate imputation by a chained equations procedure. To further characterise cases associated with bacteraemia and neurolisteriosis, we did a hierarchical clustering on principal components. Statistical analysis was done with R software (version 3.2.2). All tests were two-tailed and p values less than $0 \cdot 05$ (calculated by $\chi 2$ test, Student's t test, or Mann-Whitney test) were considered significant (appendix pp 8, 9). The study is registered at ClinicalTrials. gov, number NCT01520597.

\section{Role of the funding sources}

The funder had no role in study design, data collection, analysis and interpretation, or writing of the report. The corresponding authors had full access to the data and final responsibility for the decision to submit for publication. 


\section{RESULTS}

Of the 1063 cases notified to the NRCL between Nov 9, 2009, and July 31, 2013, 869 patients gave informed consent, of whom 818 (94\%) were included in the study (figure 1): 427 patients with bacteraemia, 252 patients with neuro- listeriosis, 107 patients with maternal-neonatal infection (table 1), and 32 patients with other forms. Follow-up was available for $99 \%$ of patients (median follow-up period 5 months [IQR 3-10]). Comparison between cases included and not included is shown in the appendix (p 10).

$35(33 \%)$ of 107 women with maternal-neonatal listeriosis were born in the Maghreb or sub-Saharan Africa, three times more than the general pregnant population in France in 2010 according to national records $(88000$ $[11 \%]$ of 832000 women, $\mathrm{p}<0 \cdot 0001) .^{14}$

Mothers of neonates with late-onset listeriosis (six of 107) had normal examination throughout pregnancy and delivery, but all others reported symptoms (101 of 107). The most frequent presentations at admission were fever with obstetric signs (contractions, labour, or abnormal fetal heart rate; 62 [62\%] of 101 cases) and fetal loss (21 [21\%] of 101 cases; figure $2 \mathrm{~A}$, table 1$)$.

Placenta and newborn gastric fluid were the most sensitive samples for microbiological diagnosis (positive in 50 [78\%] of 64 placenta samples and 52 [78\%] of 67 gastric fluid samples), whereas maternal blood cultures were positive in 47 [55\%] of 85 samples (figure 2B). In 39 (98\%) of 40 cases with maternal, placenta, and infant sample cultures, diagnosis was done with placenta or maternal blood cultures (without any other fetal-neonatal sample; figure 2C). Hypovirulent L monocytogenes clones (CC9 and CC121) were never isolated, whereas hypervirulent clones (CC1, CC2, CC4, and CC6) were identified in 70 (66\%) of 106 cases (appendix p 27). ${ }^{15}$ No strain with internalin (InlA) truncation was found, in agreement with the key contribution of this virulence factor in maternal-neonatal listeriosis. ${ }^{16}$

All pregnant women with maternal listeriosis recovered including ten $(9 \%)$ of 107 who did not receive antimicrobials (table 2). Severe sepsis, neurolisteriosis, or death was not recorded in infected mothers, but only five $(5 \%)$ of 107 had normal delivery and normal post-partum evolution (appendix p 10). 26 (24\%) of 107 mothers experienced fetal loss, 48 (45\%) of 107 premature delivery, and $22(21 \%)$ of 107 had delivery with any combination of fever or meconium fluid or abnormal fetal heart rate indicative of acute fetal distress. 40 (40\%) of 101 mothers had caesarean sections. Among 48 prematurely born infants, 20 (42\%) were born earlier than 32 weeks of gestation, 21 (44\%) between 32 and 34 weeks of gestation, and seven (15\%) between 34 and 36 weeks of gestation. Six neonates developed late-onset listeriosis 7-17 days after uneventful pregnancy and delivery. Of 107 maternal-neonatal cases, $89(83 \%)$ had major adverse outcomes: fetal death, very high prematurity $(<32$ weeks of gestation), or early or late onset disease. Fetal losses occurred before admission to hospital (21 of 26) or within 2 days after admission (five of 26). The term of pregnancy at the time of maternal listeriosis was crucial for fetal outcome, and multivariable analysis did not reveal other parameters associated with fetal loss, including maternal geographical origin, clinical features, and blood culture results (appendix p 23). Any benefit of amoxicillin-based therapy could not be demonstrated because of the low number of untreated mothers. Of the 82 neonates born alive, only ten (12\%) had normal physical evaluation with no sign of infection and did not receive antimicrobial therapy. Four prematurely born and infected neonates (born at 25-32 weeks of gestation) died as a consequence of neurological lesions attributable to infection or prematurity.

Patients with bacteraemia had a mean age of 73 (SD 14) years and those with neurolisteriosis 67 (16) years. More male patients were found when compared with the French population of the same age (59\% [398 of 679] vs $45 \%$ in the French 69-75-year-old population; p<0.0001). ${ }^{17}$ The most frequent immunosuppressive comorbidities in patients with bacteraemia or neurolisteriosis were solid organ cancer (209 [31\%] of 679) and diabetes mellitus (149 [22\%] of 679; appendix p 12). Underlying cancer was revealed in 18 (3\%) of 679 nonmaternal patients. $293(43 \%)$ of 679 patients had received corticosteroids or other immunosuppressive therapies (from one drug to 12 drugs received) over the past 5 years (appendix p 11). Ten (4\%) of 252 patients with neurolisteriosis had distinctive features that could account for inherited susceptibility to listeriosis: they were younger than 40 years, had no comorbidity or ongoing pregnancy, and no report of substantial infection before listeriosis.

Patients with bacteraemia reported at least one of the following symptoms: fever, decompensated comorbidity, diarrhoea, influenza-like symptoms, and multi-organ failure (table 1). 390 (94\%) of 415 patients had fever or tachycardia (>90 beats per min). C-reactive protein was elevated in 600 (96\%) of 627 patients and procalcitonin in $123(66 \%)$ of 186 patients. Among 252 patients with neurolisteriosis, $218(87 \%)$ had encephalitis-associated symptoms and $34(13 \%)$ had meningeal involvement without encephalitis. Meningoencephalitis was found in 
$212(84 \%)$ of 252 patients, with a mean score on the Glasgow Coma Scale of 12 (SD 3; table 1). CSF was examined in 235 patients with neurolisteriosis; all had biological signs of meningeal involvement (figure 3 ). CSF findings are shown in the appendix ( $\mathrm{p} 28$ ). Clinical involvement of the brainstem, typically considered as indicative of neurolisteriosis, was observed in only $42(17 \%)$ of 252 patients. Brain abscesses were observed in six $(2 \%)$ of 252 patients. Neurolisteriosis patients with encephalitis were older than those without encephalitis (69 [SD 14] years vs 53 [22] years, p<0·0001) and exhibited more comorbidities (median 3 [IQR 2-4] vs 1 [0-3], $\mathrm{p}<0 \cdot 0001)$. Median time interval from first symptom to diagnosis was not different in patients with neurolisteriosis with and without encephalitis (2 [IQR 1-4] days vs 2 [1-4] days, $p=0 \cdot 161)$. Lymphopenia $(<1500$ cells per $\mu \mathrm{L})$ or monocytopenia $(<200$ cells per $\mu \mathrm{L})$ were equally reported in patients with bacteraemia and patients with neurolisteriosis (table 1).

Among the 235 cases in which blood and CSF samples were cultured, 37 (16\%) had positive blood cultures only, 93 (39\%) had positive CSF culture (or PCR) only, and 105 (45\%) had both. Strains belonging to hypervirulent clonal complexes15 were more frequently identified in neurolisteriosis than bacteraemia (142 [57\%] of 250 vs 168 [40\%] of 424, p<0.0001; appendix p 27). Antimicrobial susceptibility testing of the most commonly used antibiotics did not identify resistant isolates (appendix p 29).

Amoxicillin was the most prescribed drug (table 2). Of the 679 patients with bacteraemia or neurolisteriosis, 31 (5\%) did not receive antimicrobial therapy: all died within 3 days after admission, before diagnosis (22 of 31), or 4-51 days after diagnosis (nine of 31). 32 (13\%) of 252 patients with neurolisteriosis received adjunctive dexamethasone; these patients had lower survival than patients who did not receive adjunctive dexamethasone (17 [53\%] of 32 vs 157 [73\%] of 216, p=0.037, Fisher exact test). ${ }^{18}$ Overall 3-month mortality estimated by the Kaplan- Meier method was 46\% (194 of 427 patients) for bacteraemia and 30\% (75 of 252 patients) for neurolisteriosis ( $\mathrm{p}<0.0001$; figure 4), and post-hospitalisation mortality accounted for $12 \%$ (24 of 194 patients) and $8 \%$ (six of 75 patients) of 3-month mortality in bacteraemia and neurolisteriosis, respectively. Posthospitalisation mortality was attributed to severe pre-existing comorbidities but not to late complications of listeriosis. Neurolisteriosis patients with encephalitis had three times higher mortality than those without encephalitis $(72[33 \%]$ of 218 patients vs three [9\%] of 34 patients, $p=0 \cdot 003$ ).

Persisting impairment (altered consciousness or focal signs) was reported in 79 (44\%) of 181 surviving patients with neurolisteriosis, and among them in $79(52 \%)$ of 152 of those with symptoms of encephalitis (figure 3 ). Only 99 (39\%) of 252 patients with neurolisteriosis (and among these only 68 [31\%] of 218 of those with encephalitis) survived and fully recovered at follow-up. Limb motor deficiency (12 [7\%] of 181), cerebellar symptoms (five [3\%] of 181), and eighth cranial nerve palsy (four [2\%] of 181) were the most common persisting defects in surviving patients with neurolisteriosis. Nuchal rigidity, extra- pyramidal syndrome, and third and sixth cranial nerves palsy resolved in all cases.

Parameters associated with 3-month mortality in bacteraemia and neurolisteriosis in the multivariable model were: female sex, older age, ongoing neoplasia, more than $5 \%$ weight loss, multi-organ failure, decompensated comorbidity, monocytopenia less than 200 cells per $\mu \mathrm{L}$, and high neutrophil count (table 3 ). Survival was two times lower in patients with ongoing neoplasia than without (44 [32\%] of 136 vs 366 [68\%] of 541) and among patients with aggravation of any pre-existing organ dysfunction than without (85 [35\%] of 240 vs 325 [74\%] of 439). Survival was also three times lower in patients with multi-organ failure than without (23 [19\%] of 124 vs $387[70 \%]$ of $555, \mathrm{p}<0 \cdot 0001)$.

Parameters associated with survival among patients with bacteraemia and neurolisteriosis were: influenza-like symptoms, administration of anti-listeria beta-lactam, or cotrimoxazole or an aminoglycoside. Treated patients receiving anti-listeria beta-lactam had a 3.3 times higher survival than those who did not (402 [66\%] of 608 vs eight $[20 \%]$ of $40, p<0 \cdot 0001)$. Those receiving amino- glycoside also had increased survival (292 [69\%] of 423 vs 118 [52\%] of 225, p<0.0001). Duration of amoxicillin- aminoglycoside combination therapy ( $>3$ days) had an independent protective effect when added to the multivariable model ( $>3$ days vs 0 days, odds ratio [OR] $0 \cdot 35$ [95\% CI $0 \cdot 22-0 \cdot 56], \mathrm{p}<0 \cdot 0001)$. In multivariable analysis focusing on neurolisteriosis, positive blood cultures and adjunctive dexamethasone prescription (prescribed within the first $24 \mathrm{~h}$ after admission) were associated with 3-month mortality (table 3). Survival was significantly lower in patients with positive blood cultures than in patients without positive blood cultures (95 [60\%] of 158 vs 82 [87\%] of 94, p<0.0001). 17 (52\%) of 32 patients receiving adjunctive dexamethasone survived and 157 (73\%) of 216 of those who did not receive adjunctive dexamethasone survived $(\mathrm{p}=0 \cdot 024)$, although both groups had similar mean Glasgow Coma Scale scores (12 [SD 3 ] in both) and similar proportions with encephalitis (28 [86\%] of 32 vs 185 [84\%] of 220). Presence of encephalitis-associated signs was the strongest parameter associated with persistent neurological impairment in neurolisteriosis cases (78 [52\%] of 149 vs one [3\%] of 32). 
To assess the relevance of bacteraemia versus neuro- listeriosis (bacteraemic or not) classification, a hierarchical clustering was done (appendix p 9). The analysis showed three clusters, one almost exclusively neurolisteriosis cases (cluster 3), and two other clusters (clusters 1 and 2) comprising predominantly bacteraemia cases as well as neurolisteriosis cases with a higher rate of positive blood culture and poorer outcomes than in cluster 3: cluster 1 comprised patients with iatrogenic immunosuppression and cluster 2 older patients (appendix p 30). In agreement with these results, survival of patients with bacteraemic neurolisteriosis was significantly lower than in patients with non-bacteraemic neurolisteriosis, but in the range of those with bacteraemia alone (figure 4B).

\section{DISCUSSION}

The quasi-exhaustiveness of the National Surveillance System for listeriosis in France made it possible to do the first prospective clinical study on listeriosis. We found that more than $80 \%$ of infected mothers experienced major fetal or neonatal complications and only $39 \%$ of patients with neurolisteriosis survived and fully recovered. Fetal losses occurred at less than 29 weeks of gestation and within 2 days of hospital admission and new factors independently associated with mortality were identified. Our data show the deleterious effect of adjunctive dexamethasone in neurolisteriosis, and argue for the use of beta-lactam and gentamicin or cotrimoxazole over other anti- microbials for bacteraemia and neurolisteriosis.

The strengths of this study are its exhaustiveness at inclusion and follow-up ( $99 \%$ of patients had follow-up data available), and the wide array of data collected (more than 500 items) for each patient. It is unusual to capture an almost complete dataset associated with a given pathogen on such a large scale in space and time. MONALISA provides crucial new data on listeriosis.

An unexpectedly high proportion of mothers with listeriosis report an African origin (33\%), associated in France with lower socioeconomic conditions, mirroring the increased incidence of maternal-neonatal listeriosis in US Hispanic and UK ethnic minorities. ${ }^{19-21}$ Since higher fecundity rates cannot account for this phenomenon, ${ }^{14}$ the role of specific dietary habits (as described in Hispanic minorities in the USA ${ }^{21}$ ) remains to be studied, along with the contribution of listeriosis awareness, health-seeking behaviour, and health-care system access in this population. The study highlights the challenges of maternal diagnosis. Mothers had a large array of symptoms, none of them sensitive for the diagnosis of listeriosis. Furthermore, symptoms might vary largely. Fever, for instance, has been reported in $16-65 \%$ of mothers. ${ }^{5,22}$ Maternal blood cultures (on which diagnosis before delivery relies) were negative in $45 \%$, arguing for the maintenance of anti-listeria therapy despite negative maternal blood cultures where listeriosis is suspected. This finding reflects previous retrospective analyses that report only a $45 \%$ positivity of maternal blood cultures. ${ }^{5}$ The prospective design of the study allowed a precise evaluation of fetal outcomes, with $83 \%$ of infected mothers experiencing major fetal or neonatal complications, which is worse than has been estimated in retrospective studies. ${ }^{3,5}$ Term at onset is the crucial outcome parameter. Risk of fetal death is minimal when listeriosis occurs after 29 weeks of gestation, and whatever the term of pregnancy after the first 2 days of admission to hospital. This 29 week cutoff is slightly lower than previously reported, with fetal losses even in late pregnancies being rarely reported.10 This finding might reflect an increased medical awareness, in the context of French recommendations on the empiric prescription of amoxicillin when listeriosis is suspected during pregnancy. ${ }^{23}$

The MONALISA study identified the clinical features and prognosis of non-maternal listeriosis with an unprecedented precision. Regarding neurolisteriosis, our findings show the lack of sensitivity of brainstem involvement, despite its frequent reporting in retrospective studies. ${ }^{4}$ This result emphasises the rarity of isolated encephalitis, in agreement with previous data reporting the low frequency of "isolated brain abscesses/cerebritis". ${ }^{4}$ Long-term neurological sequelae were observed in $44 \%$ of surviving patients with neurolisteriosis, reflecting that reported in the Dutch cohort of 92 patients with neurolisteriosis and more than that reported for bacterial meningitis $(18 \%)$ and infectious encephalitis $(33 \%){ }^{24-26}$ That only $33 \%$ of patients with L monocytogenes encephalitis survived without persisting impairment further underscores the severity of neuro- listeriosis. New independent factors associated with mortality in bacteraemia and neurolisteriosis were identified. The strongest factors are ongoing cancer, multi- organ failure, decompensated comorbidity, monocytopenia, and also concomitant bacteraemia for neurolisteriosis. Only older age and malignancies were previously identified; other features such as alcoholism, antacid uptake, corticosteroid medication, chronic lung or kidney disease, blood transfusion, and Asian and Hispanic ethnicity recognised as risk factors for death in smaller cohorts were not shown in our study. ${ }^{8,9,27}$

Neurolisteriosis in human beings is a blood-borne infection. Positive blood cultures at the time of diagnosis could reflect higher bacterial load and weaker host defences, leading to higher mortality, as shown in other opportunistic infections (eg, cryptococcosis). ${ }^{28}$ This hypothesis might also account for the counter-intuitive observation of poorer outcome in bacteraemia-only than with neurolisteriosis $(46 \%$ vs $30 \%$ for 3 -month mortality). Multivariate analyses suggest that beta-lactams and aminoglycosides or cotrimoxazole could improve survival more than other antibiotics in bacteraemia and neurolisteriosis, independently of any associated factor. Whereas smaller size studies and retrospective studies suggest a deleterious effect of amino- glycosides, our 
results, although not from a randomised trial, could be considered the strongest available evidence so far arguing for the use of combined amoxicillin- gentamicin as first-line therapy for invasive listeriosis. ${ }^{29,30}$ The findings are in line with the documented in-vitro synergy and bactericidal effect of amoxicillin plus gentamicin on $L$ monocytogenes - amoxicillin alone is only weakly bactericidal. ${ }^{31}$ Whether the relatively short mean duration of bacteraemia treatment in our study (17 days) accounts, at least in part, for the poor prognosis remains to be determined.

Multivariate analyses showed for the first time a significantly reduced survival in patients with neuro- listeriosis treated with adjunctive dexamethasone. This deleterious effect had been suggested before in the Dutch cohort of 92 patients with neurolisteriosis ${ }^{26}$ that showed a trend, although non-significant, towards poorer outcome among patients with neurolisteriosis treated with dexamethasone. Even though our result is not from a clinical trial and the number of treated patients was small $(n=32)$, it suggests that dexamethasone should be avoided in the treatment of neurolisteriosis. ${ }^{18}$ The identification of young patients with neurolisteriosis and without an identified comorbidity could suggest a genetic susceptibility to listeriosis, although one might not also exclude the possibility of a massive inoculum. ${ }^{21}$

Our study has limitations. First, all screened patients could not be included. The outcome of excluded neurolisteriosis cases was worse than included cases, with a higher mortality rate: this limitation suggests that the actual burden of listeriosis, at least for neurological infections, might be even higher than reported here. Potential biases were addressed by doing multivariable regressions to control for confounding factors. Multiple imputation was used for multivariable models to limit bias arising from missing data. No adjustment for multiple testing was done in our exploratory approach. The proportional hazards assumption was tested and no violation of the proportional assumption was found. Although dexamethasone was given within $24 \mathrm{~h}$ of management in all cases, the precise timing of administration (before or after the first antibiotic dose) was not recorded. For these reasons, confirmatory studies on external cohorts are needed to support our results.

In conclusion, this study highlights much more serious outcomes of listeriosis than has been reported to date, with greater than $80 \%$ of infected mothers experiencing major fetal or neonatal complications. Furthermore, only $39 \%$ of study shows a deleterious effect of adjunctive dexa- methasone in neurolisteriosis, and argues for the use of beta-lactam, gentamicin, and co-trimoxazole over other antibiotics for bacteraemia and neurolisteriosis. Given the practical difficulties in doing clinical trials in listeriosis, these results constitute a unique basis from which clinical practice might evolve to better diagnose and treat this deadly infection. 


\section{Tables and figures legends}

Table 1. Characteristics of the study population

Data are mean (SD), median (IQR), n (\%), or n/N (\%), unless otherwise stated. *Mann-Whitney test. $\dagger \chi 2$ test. $\$$ Immunosuppressive comorbidities included: daily alcohol intake more than three drinks per day, cirrhosis, diabetes mellitus, end-stage renal disease, solid organ cancer, haematological malignancy, haemopoietic stemcell transplantation, solid organ transplantation, asplenia, pre-existing neutropenia, pre- existing lymphopenia, HIV infection, inflammatory bowel diseases, inflammatory rheumatic disorders, other autoimmune diseases, congenital immune deficiency, age older than 70 years, prescription of corticosteroids, or other immunosuppressive therapies in the past 5 years. §Fisher exact test.

Table 2. Summary of treatments and outcomes for patients with maternal listeriosis, bacteraemia, and neurolisteriosis

Data are mean (SD), median (IQR), $\mathrm{n}(\%)$, or $\mathrm{n} / \mathrm{N}(\%)$, unless otherwise stated. Data were not available for all patients in the bacteraemia and neurolisteriosis groups where indicated. *Two mothers received erythromycin, and another two received ineffective cephalosporin-based therapy. †Treatment data were available for 251 patients with neurolisteriosis. $¥$ Of the ten mothers who did not receive antibiotic therapy, six were women whose infant was diagnosed with late onset disease. §Two deaths were censored because patients died more than 3 months after diagnosis but in hospital. IInfections included severe sepsis or septic shock $(n=4)$, pneumonia $(n=8)$, undocumented sepsis and bacteraemia $(n=2$ each), pyelonephritis, cellulitis, bile tract infection, otitis media, colitis, endocarditis, endovascular infection $(n=1$ each), and opportunistic infections (cytomegalovirus-related encephalitis, aspergillosis, and nocardiosis, $n=1$ each). IIAll recurrences were bacteraemia.

Table 3. Multivariable logistic regression analyses of factors associated with 3-month mortality for patients with bacteremia and neurological infections and with persisting neurological impairment for patients with neurological infections

Results from univariable analyses are presented in appendix (p12). * 269 died within 3 months after diagnosis $(269 / 679,40 \%) .^{\dagger}$ Adjusted odds ratios calculated from the multivariable model after imputation of missing data. CI were estimated using Rubin’s rules. ${ }^{\star}$ Beta-lactams lacking activity towards Listeria spp. were excluded (i.e. oxacillin and cephalosporins). ${ }^{\S} 79$ patients reported persisting impairment (79/181, 44\%). " Encephalitis symptoms included at least one of the following signs, with no alternative cause identified: altered consciousness (score on Glasgow Coma Scale $<15$ ), seizures, new onset of focal neurological sign and any abnormality on electroencephalography consistent with encephalitis (appendix, p9). 


\section{Figure 1: Patient selection}

*Patients not meeting the inclusion criteria were patients with no French health-care insurance, according to French ethics legislation, or patients for whom written consent could not be obtained (either from them of from their next of kin). $† 17$ patients were classified as patients with neurolisteriosis although they did not have lumbar puncture performed; all had positive blood cultures, all reported fever, with either neck stiffness evocative of meningitis $(n=4)$, coma $(n=3)$, coma and neck stiffness $(n=2)$, neurological signs (homonymous hemianopia, cerebellous syndrome, and seizures, $n=1$ each), brain abscess $(n=1)$, or neck stiffness and focal neurological signs (aphasia and pyramidal syndrome, pyramidal syndrome alone, bilateral motor deficiency, and hemiparesis, $\mathrm{n}=1$ each); these symptoms were regressive in all cases under antibiotic therapy, with no alternative cause identified; in all cases, clinicians considered neurological signs as the manifestation of neurolisteriosis, and prescribed high-dose antibiotic therapy. $\$$ Focal infections involved the peritoneal fluid $(n=13)$, bones and joints $(n=8)$, pleural space, cardiovascular infections $(n=3$, each), urinary tract infections $(n=2)$, pneumonia, biliary tract, and adenitis $(\mathrm{n}=1$, each).

\section{Figure 2. Clinical and biological presentation of maternal-neonatal listeriosis}

(A) Pattern of maternal and obstetric symptoms for maternal-neonatal presentation at admission. *Mothers of the six neonates with late onset disease had no symptoms and are not included in this panel; mean term at the diagnosis of listeriosis was 30 (SD 8) weeks of gestation. (B) Frequency of positive samples among the 107 maternal-neonatal cases. †Other samples included various organs collected at autopsy. (C) Distribution of culture-positive samples in the 40 maternal-neonatal cases with maternal blood cultures, placenta, and infant samples collected (green, maternal blood cultures [ $n=22]$; pink, fetal or neonatal samples [ $n=31]$; blue, placenta cultures $[n=30])$.

\section{Figure 3. Clinical and biological presentation of neurolisteriosis}

(A) Clinical features of neurolisteriosis. *Study population included the 252 patients with neurolisteriosis. $\dagger$ Brainstem involvement was defined by the presence of any cranial nerve lesion (except the first nerve). \$Cerebrospinal fluid (CSF) examination was done in 235 patients with neurolisteriosis; opening pressures (intracranial presssure of the CSF measured during lumbar puncture) were not assessed. §Polymorphonuclear cells to nucleated cells ratio was assessed in 231 patients. JCSF to blood glucose ratio was determined for 197 patients. ILListeria monocytogenes-specific PCR results for CSF were positive in ten of 16 tested samples: one of one with a 16s amplification, eight of 14 with a $h l y$ amplification, and one of one other non-specified local amplification technique; of the three patients with PCR-positive CSF and concomitant negative cultures, two had antibiotics when lumbar puncture was done. (B) Schematic representation of clinical and microbiological baseline features of neurolisteriosis. Left: green, clinical encephalitis $(n=218)$; blue, clinical meningitis $(n=163)$; red, CSF abnormality among patients with neurolisteriosis $(n=235)$. Right: red, positive blood culture $(n=142)$; blue, positive CSF culture $(n=197)$. ${ }^{*}$ Encephalitis was defined by the presence of at least one of the following symptoms with no alternative cause than listeriosis identified: altered consciousness (score on Glasgow Coma Scale $<15$ ), seizures, new onset of neurological symptoms, and abnormality on electroencephalography consistent with encephalitis (appendix). ††CSF abnormality was defined by at least one of the following conditions: a nucleated cells count of more than 4 per $\mu \mathrm{L}$, protein count of less than $0.5 \mathrm{~g} / \mathrm{L}$, or the presence of Listeria monocytogenes in the cerebrospinal fluid by culture or PCR. $\$ \$ 11$ patients and six patients with clinical encephalitis but no alteration of the CSF had no lumbar puncture performed.

\section{Figure 4. Survival of patients with maternal listeriosis, bacteraemia, or neurolisteriosis}

(A) Kaplan-Meier plot of the time to death of patients with maternal listeriosis, bacteraemia, or neurolisteriosis. Survival is significantly higher in patients with neurolisteriosis than in patients with bacteraemia (hazard ratio [HR] 0.54 [95\% CI 0.41-0.69], p<0.0001). No test was done for maternal patients because there was no death in this group. (B) Kaplan-Meier plot of the time to death of patients with bacteraemia, neurolisteriosis and bacteraemia, and neurolisteriosis without bacteraemia. Survival is significantly higher in cases of neurolisteriosis

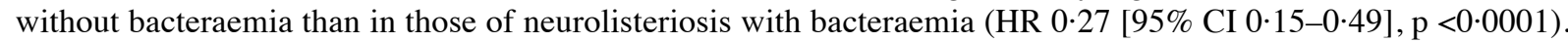
Survival is significantly higher in neurolisteriosis without bacteraemia than in bacteraemia alone (HR 0.21 [95\% CI $0 \cdot 12-0 \cdot 36], p<0 \cdot 0001)$. Survival is not significantly higher in neurolisteriosis with bacteraemia than in bacteraemia alone (HR 0.77 [95\% CI 0.59-1·00], $\mathrm{p}=0 \cdot 054)$. 


\section{Acknowledgements}

We thank the Clinical Research Unit Paris Centre, in particular Irma Pelaez, Kelly Cheung, Camille Levalois, Thierry Cachina, Magatte Fall, Gabrielle Coulpier, Laurence Lecomte, Prissile Bakouboula, and Jean-Marc Treluyer. We also thank Edith Laurent (French Public Health Agency) and Saadia Jerbi (Necker-Enfants Malades University Hospital) for data collection.

\section{Conflicts of interest}

We declare no competing interests.

\section{Contributors}

$\mathrm{CC}, \mathrm{AL}$ and ML did the literature search.

$\mathrm{CC}$ and ML conceived the study, together with VG, PR and OL.

$\mathrm{CC}, \mathrm{AL}, \mathrm{BC}, \mathrm{BP}, \mathrm{BH}, \mathrm{AL}, \mathrm{PT}$ and HBD did the data collection.

EP, AM, MM and CC did the statistical analyses and all authors helped to interpret the data.

$\mathrm{CCW}$ and ML wrote the manuscript and all authors reviewed the manuscript. 


\section{REFERENCES}

\section{References}

1. Scallan E, Hoekstra RM, Angulo FJ, et al. Foodborne illness acquired in the United States-major pathogens. Emerg Infect Dis 2011; 17: 7-15.

2. Maertens de Noordhout C, Devleesschauwer B, Angulo FJ, et al.The global burden of listeriosis: a systematic review and meta-analysis. Lancet Infect Dis 2014; 14: 1073-82.

3. McLauchlin J. Human listeriosis in Britain, 1967-85, a summary of 722 cases. 1. Listeriosis during pregnancy and in the newborn. Epidemiol Infect 1990; 104: 181-89.

4. Mylonakis E, Hohmann EL, Calderwood SB. Central nervous system infection with Listeria monocytogenes. 33 years' experience at a general hospital and review of 776 episodes from the literature. Medicine 1998; 77: 313-36.

5. Mylonakis E, Paliou M, Hohmann EL, Calderwood SB, Wing EJ. Listeriosis during pregnancy: a case series and review of 222 cases. Medicine 2002; 81: 260-69.

5. Goulet V, Hebert M, Hedberg C, et al. Incidence of listeriosis and related mortality among groups at risk of acquiring listeriosis. Clin Infect Dis 2012; 54: 652-60.

7. Campbell DM. Human listeriosis in Scotland 1967-1988. J Infect 1990; 20: 241-50.

8. Gerner-Smidt P, Ethelberg S, Schiellerup P, et al. Invasive listeriosis in Denmark 1994-2003: a review of 299 cases with special emphasis on risk factors for mortality. Clin Microbiol Infect 2005; 11: 618-24.

9. Guevara RE, Mascola L, Sorvillo F. Risk factors for mortality among patients with nonperinatal listeriosis in Los Angeles County, 1992-2004. Clin Infect Dis 2009; 48: 1507-15.

10. Girard D, Leclercq A, Laurent E, Lecuit M, de Valk H, Goulet V. Pregnancy-related listeriosis in France, 1984 to 2011 , with a focus on 606 cases from 1999 to 2011. Euro Surveill 2014; 19 (38).

11. de Valk H, Tourdjman M, Leclercq A, et al. Changes in epidemiology and surveillance of listeriosis in France. International Symposium on Problems of Listeriosis; Paris; June 15, 2016. Institut Pasteur. Abstract 8. http://www.isopol2016.org/ (accessed Jan 10, 2017).

12. von Elm E, Altman DG, Egger M, Pocock SJ, Gotzsche PC, Vandenbroucke JP. The Strengthening the Reporting of Observational Studies in Epidemiology (STROBE) statement: guidelines for reporting observational studies. Lancet 2007; 370: $1453-57$.

13. Moura A, Criscuolo A, Pouseele H, et al. Whole genome-based population biology and epidemiological surveillance of Listeria monocytogenes. Nat Microbiol 2016; 2: 16185.

14. Insee. Statistiques d'état civil sur les naissances en 2011. 2012.

http://www.insee.fr/fr/themes/document.asp?ref_id=sd20111 (accessed June 4, 2015).

15. Maury MM, Tsai YH, Charlier C, et al. Uncovering Listeria monocytogenes hypervirulence by harnessing its biodiversity. Nat Genet 2016; 48: 308-13.

16. Lecuit M, Nelson DM, Smith SD, et al. Targeting and crossing of the human maternofetal barrier by Listeria monocytogenes: role of internalin interaction with trophoblast E-cadherin. Proc Natl Acad Sci USA 2004; 101: $6152-57$.

17. Insee. Pyramide des âges au 1er janvier 2012. 2013. http://www.insee. fr/fr/ppp/bases-de-donnees/donneesdetaillees/bilan-demo/fichiers- xls/pyramide-des-ages-2012.xls\%5D (accessed June 3, 2015).

18. Tunkel AR, Hartman BJ, Kaplan SL, et al. Practice guidelines for the management of bacterial meningitis. Clin Infect Dis 2004; 39: 1267-84.

19. Lombardo P, Pujol J. Niveau de vie et pauvreté des immigrés en 2007. 2007.

http://www.insee.fr/fr/themes/document.asp?reg_id=0\&ref_id=REVPMEN10d (accessed Feb 6, 2016).

20. Mook P, Grant KA, Little CL, Kafatos G, Gillespie IA. Emergence of pregnancy-related listeriosis amongst ethnic minorities in England and Wales. Euro Surveill 2010; 15: 17-23. 
21. Pouillot R, Hoelzer K, Jackson KA, Henao OL, Silk BJ. Relative risk of listeriosis in Foodborne Diseases Active Surveillance Network (FoodNet) sites according to age, pregnancy, and ethnicity. Clin Infect Dis 2012; 54 (suppl 5): S40510.

22 ; Elinav H, Hershko-Klement A, Valinsky L, et al. Pregnancy-associated listeriosis: clinical characteristics and geospatial analysis of a 10-year period in Israel. Clin Infect Dis 2014; 59: 953-61.

23. Charlier C, Goffinet F, Azria E, Leclercq A, Lecuit M. Inadequate management of pregnancy-associated listeriosis: lessons from four case reports. Clin Microbiol Infect 2014; 20: 246-49.

24. van de Beek D, de Gans J, Spanjaard L, Weisfelt M, Reitsma JB, Vermeulen M. Clinical features and prognostic factors in adults with bacterial meningitis. $N$ Engl J Med 2004; 351: 1849-59.

25. Mailles A, De Broucker T, Costanzo P, Martinez-Almoyna L, Vaillant V, Stahl JP. Long-term outcome of patients presenting with acute infectious encephalitis of various causes in France. Clin Infect Dis 2012; 54: 1455-64.

26. Koopmans MM, Brouwer MC, Bijlsma MW, et al. Listeria monocytogenes sequence type 6 and increased rate of unfavorable outcome in meningitis: epidemiologic cohort study. Clin Infect Dis 2013; 57: 247-53.

27. Mook P, Patel B, Gillespie IA. Risk factors for mortality in non-pregnancy-related listeriosis. Epidemiol Infect 2012; 140: $706-15$

28. Dromer F, Mathoulin-Pelissier S, Launay O, Lortholary O. Determinants of disease presentation and outcome during cryptococcosis: the CryptoA/D Study. PLoS Med 2007; 4: e21.

29. Amaya-Villar R, Garcia-Cabrera E, Sulleiro-Igual E, et al. Three-year multicenter surveillance of community-acquired Listeria monocytogenes meningitis in adults. BMC Infect Dis 2010; 10: 324.

30. Mitja O, Pigrau C, Ruiz I, et al. Predictors of mortality and impact of aminoglycosides on outcome in listeriosis in a retrospective cohort study. J Antimicrob Chemother 2009; 64: 416-23.

31. Hof H. Listeriosis: therapeutic options. FEMS Immunol Med Microbiol 2003; 35: 203-05. 


\begin{tabular}{|c|c|c|c|c|c|}
\hline Characteristics* & $\begin{array}{l}\text { Maternal } \\
(\mathrm{N}=107)\end{array}$ & $\begin{array}{l}\text { Bacteremia } \\
(\mathrm{N}=427)\end{array}$ & $\begin{array}{l}\text { Neurolisteriosis } \\
\quad(\mathbf{N}=\mathbf{2 5 2})\end{array}$ & $\begin{array}{c}\text { Difference in means or } \\
\text { proportions between } \\
\text { bacteremia and } \\
\text { neurolisteriosis }(95 \% \mathrm{CI})\end{array}$ & $\begin{array}{c}\text { p-value } \\
\text { bacteremia } \\
\text { versus } \\
\text { neurolisteriosis } \\
\end{array}$ \\
\hline Age - years (no evaluated) & $30 \pm 5(107)$ & $73 \pm 14(427)$ & $67 \pm 16(252)$ & $6(4 ; 8)$ & $<0 \cdot 001^{*}$ \\
\hline Male gender - no $/$ no $\cdot$ evaluated $(\%)$ & - & $246 / 427(58)$ & $152 / 252(60)$ & $-3(-10 ; 5)$ & $0 \cdot 489^{\S}$ \\
\hline Associated comorbidities (no evaluated) & $(107)$ & (427) & $(252)$ & & \\
\hline Median number of comorbidities & $0[0 ; 1]$ & $3[2 ; 5]$ & $3[1 ; 4]$ & $0 \cdot 7(0 \cdot 4 ; 1 \cdot 0)$ & $<0 \cdot 001^{\ddagger}$ \\
\hline Median number of immunosuppressive comorbidities ${ }^{\dagger}$ & $0[0 ; 0]$ & $2[1 ; 4]$ & $2[1 ; 3]$ & $0 \cdot 5(0 \cdot 3 ; 0 \cdot 8)$ & $<0 \cdot 001^{\ddagger}$ \\
\hline At least one immunosuppressive comorbidity & $7 / 107(7)$ & $416 / 427(97)$ & $216 / 252(86)$ & $12(7 ; 16)$ & $<0 \cdot 001^{\S}$ \\
\hline Median time interval from first symptom to diagnosis -days (no evaluated) & $1[0 ; 3](98)$ & $2[0 ; 6](369)$ & $2[1 ; 4](243)$ & $1 \cdot 6(0 \cdot 3 ; 3 \cdot 0)$ & $0 \cdot 684^{\ddagger}$ \\
\hline Temperature $>37 \cdot 7^{\circ} \mathrm{C}-$ no $\cdot /$ no $\cdot$ evaluated $(\%)$ & $83 / 107(78)$ & $370 / 427(87)$ & $244 / 252(97)$ & $-10(-14 ;-6)$ & $<0 \cdot 001^{\S}$ \\
\hline Mean maximal body temperature $\left({ }^{\circ} \mathrm{C}\right)($ no $\cdot$ evaluated) & $39 \pm 1(100)$ & $39 \pm 1(389)$ & $40 \pm 1(246)$ & $-0 \cdot 5(-0 \cdot 6 ;-0 \cdot 3)$ & $<0 \cdot 001^{\S}$ \\
\hline Flu-like symptoms (muscle and joint pains, chills) & 35/107 (33) & $87 / 427(20)$ & $69 / 252(27)$ & $-7 \cdot 0(-13 \cdot 7 ;-0 \cdot 3)$ & $0 \cdot 036^{\S}$ \\
\hline Systolic blood pressure $<90 \mathrm{~mm} \mathrm{Hg}-$ no $/$ no evaluated (\%) & 1/93 (1) & $27 / 390(7)$ & $8 / 247(3)$ & $3 \cdot 7(0 \cdot 3 ; 7 \cdot 0)$ & $0 \cdot 047^{\S}$ \\
\hline Diarrhea ( $>3$ stools / day) & $8 / 107(8)$ & $92 / 427(22)$ & $38 / 252(15)$ & $6(1 ; 12)$ & $0 \cdot 039^{\S}$ \\
\hline Septic chock & $0 / 107(0)$ & $5 / 427(1)$ & $5 / 252(2)$ & $-1(-3 ; 1)$ & $0 \cdot 512^{\Phi \S}$ \\
\hline \multicolumn{6}{|l|}{ Score on Glasgow Coma Scale } \\
\hline Mean (no· evaluated) & $15 \pm 0(107)$ & $14 \pm 2(420)$ & $12 \pm 3(244)$ & $2 \cdot 1(1 \cdot 7 ; 2 \cdot 4)$ & $<0 \cdot 001^{\ddagger}$ \\
\hline$<14$ (change in mental status) - no $/$ no evaluated (\%) & $1 / 107(1)$ & $57 / 420(14)$ & $121 / 244(50)$ & $-36(-43 ;-29)$ & $<0 \cdot 001^{\S}$ \\
\hline$<8$ (coma) - no $\cdot /$ no $\cdot$ evaluated $(\%)$ & $0 / 107(0)$ & $12 / 420(3)$ & $24 / 244(10)$ & $-7(-11 ;-3)$ & $<0 \cdot 001^{\S}$ \\
\hline \multicolumn{6}{|l|}{ Blood cultures } \\
\hline Performed & $85 / 107(79)$ & 427/427(100) & $252 / 252(100)$ & - & - \\
\hline Positive & $47 / 85(55)$ & $427 / 427(100)$ & $158 / 252(63)$ & $37(31 ; 43)$ & $<0 \cdot 001^{\S}$ \\
\hline \multicolumn{6}{|l|}{ Blood count } \\
\hline Mean blood leucocytes count $/ \mathrm{mm}^{3}$ (no· evaluated) & $14830 \pm 7410(98)$ & $10920 \pm 10740(420)$ & $12890 \pm 9130(251)$ & $-1960(-3560 ;-370)$ & $<0 \cdot 001^{\ddagger}$ \\
\hline Mean blood polymorphonuclear cells / $/ \mathrm{mm}^{3}$ (no· evaluated) & $12350 \pm 9800(88)$ & $8400 \pm 6710(408)$ & $9920 \pm 5880(248)$ & $-1530(-2540 ;-510)$ & $<0 \cdot 001^{\ddagger}$ \\
\hline Blood leucocytes count $<10^{4} / \mathrm{mm}^{3}-$ no $/$ no evaluated (\%) & 28/98 (29) & $239 / 420(57)$ & $93 / 251(37)$ & $20(12 ; 27)$ & $<0 \cdot 001$ \\
\hline Lymphopenia $<1500 / \mathrm{mm}^{3}-$ no$\cdot /$ no $\cdot$ evaluated $(\%)$ & $36 / 87(41)$ & $342 / 408(84)$ & 196/247 (79) & $4(-2 ; 11)$ & $0 \cdot 148^{\S}$ \\
\hline Monocytopenia $<200 / \mathrm{mm}^{3}-$ no $/$ no $\cdot$ evaluated (\%) & $1 / 59(2)$ & $49 / 358(14)$ & $24 / 230(10)$ & $3(-2 ; 9)$ & $0 \cdot 243^{\S}$ \\
\hline Monocytosis $>1000 / \mathrm{mm}^{3}-$ no $/$ no $\cdot$ evaluated $(\%)$ & $10 / 59(17)$ & $59 / 358(16)$ & $65 / 230(28)$ & $-12(-19 ;-5)$ & $<0 \cdot 001^{\S}$ \\
\hline \multicolumn{6}{|l|}{ Blood chemical tests } \\
\hline C-reactive protein $-\mathrm{mg} /$ liter - no· evaluated & $102 \pm 74(91)$ & $115 \pm 87$ (394) & $113 \pm 90(233)$ & $2(-12 ; 16)$ & $0 \cdot 571^{*}$ \\
\hline C-reactive protein $<10 \mathrm{mg} /$ liter - no $/$ no $\cdot$ evaluated (\%) & $0 / 91(0)$ & $10 / 394(3)$ & $17 / 233(7)$ & $-5(-8 ;-1)$ & $0 \cdot 005^{\S}$ \\
\hline Serum procalcitonin $-\mathrm{ng} / \mathrm{ml}$ (no· evaluated) & $0 \cdot 2[0 \cdot 1 ; 0 \cdot 2](5)$ & $0 \cdot 9[0 \cdot 3 ; 6 \cdot 8](71)$ & $1 \cdot 4[0 \cdot 3 ; 6 \cdot 2](115)$ & $-1(-7 ; 6)$ & $0 \cdot 515^{\ddagger}$ \\
\hline Serum procalcitonin $<0 \cdot 5 \mathrm{ng} / \mathrm{ml}-$ no $/$ no $\cdot$ evaluated (\%) & $5 / 5(100)$ & $27 / 71(38)$ & $36 / 115(31)$ & $7(-7 ; 21)$ & $0 \cdot 347^{\S}$ \\
\hline
\end{tabular}




\begin{tabular}{|c|c|c|c|}
\hline A. Treatment characteristics according to the form of infection* & $\begin{array}{c}\text { Maternal }^{+} \\
(\mathbf{N}=107)\end{array}$ & $\begin{array}{c}\text { Bacteremia } \\
(\mathrm{N}=427)\end{array}$ & $\begin{array}{c}\text { Neurolisteriosis } \\
(\mathbf{N}=\mathbf{2 5 1})\end{array}$ \\
\hline Mean number of antibiotics & $1 \cdot 7 \pm 1 \cdot 1$ & $2 \cdot 3 \pm 1 \cdot 4$ & $3 \cdot 2 \pm 1 \cdot 4$ \\
\hline Median duration of antibiotics in days & $15[8 ; 21]$ & $17[9 ; 23]$ & $22[18 ; 25]$ \\
\hline Amoxicillin - no $(\%)$, median duration in days & $91(85), 15[9 ; 22]$ & $349(82), 16[11 ; 22]$ & $244(97), 22[15 ; 23]$ \\
\hline Imipenem - no $(\%)$, median duration in days & $0(0)$ & $13(3), 8[4 ; 16]$ & $10(4), 7[5 ; 23]$ \\
\hline Gentamicin - no $(\%)$, median duration in days & $32(30), 3[2 ; 4]$ & $205(48), 4[3 ; 6]$ & $200(79), 7[4 ; 8]$ \\
\hline Cotrimoxazole - no. $(\%)$, median duration in days & $0(0)$ & $49(12), 11[6 ; 22]$ & $42(17), 20[14 ; 30]$ \\
\hline Rifampicin - no $(\%)$, median duration in days & $0(0)$ & $6(1), 21[11 ; 25]$ & $3(1), 22[19 ; 23]$ \\
\hline Vancomycin - no $(\%)$, median duration in days & $1(1), 3[3 ; 3]$ & $19(4), 4[2 ; 11]$ & $24(10), 2[2 ; 6]$ \\
\hline Linezolid - no $(\%)$, median duration in days & $1(1), 15[15 ; 15]$ & $5(1), 13[10 ; 15]$ & $4(2), 14[13 ; 21]$ \\
\hline Amoxicillin+Gentamicin - no $(\%)$, median duration in days & $30(28), 3[2 ; 4]$ & $170(40), 4[3 ; 6]$ & $192(76), 7[4 ; 8]$ \\
\hline Amoxicillin + Cotrimoxazole - no $(\%)$, median duration in days & $0(0)$ & $33(8), 11[6 ; 22]$ & $37(15), 20[14 ; 30]$ \\
\hline No treatment - no $\cdot(\%)$ & $10(9)^{*}$ & $30(7)$ & $1(1)$ \\
\hline Dexamethasone - no $(\%)$ & - & - & $32(13)$ \\
\hline B. Outcome according to the form of infection & $\begin{array}{c}\text { Maternal } \\
(\mathbf{N}=107)\end{array}$ & $\begin{array}{c}\text { Bacteremia } \\
(\mathrm{N}=427)\end{array}$ & $\begin{array}{c}\text { Neurolisteriosis } \\
(\mathbf{N}=\mathbf{2 5 2})\end{array}$ \\
\hline Intensive care unit management - no $/$ no $\cdot$ evaluated (\%) & 2/107 (2) & $89 / 427(21)$ & $152 / 252(60)$ \\
\hline Median hospital-stay in days & $6[4 ; 11]$ & $15[7 ; 24]$ & $23[15 ; 33]$ \\
\hline Mechanical ventilation - no / no evaluated (\%) & $0 / 107(0)$ & $43 / 427(10)$ & $83 / 252(33)$ \\
\hline Multi-organ failure - no $/$ no· evaluated (\%) & 0/107 (0) & $75 / 427(18)$ & $49 / 252(19)$ \\
\hline Aggravation of any pre existing organ dysfunction - no $/$ no evaluated (\%) & $0 / 107(0)$ & $182 / 427(43)$ & $58 / 252(23)$ \\
\hline 3-month death - no $/$ no $\cdot$ evaluated (\%) & $0 / 107(0)$ & $194 / 427(45)$ & $75 / 252(30)$ \\
\hline Median interval from diagnosis to 3-month death in days & - & $10[3 ; 23]$ & $14[5 ; 30]$ \\
\hline 3-month in-hospital death - no $/$ no evaluated $(\%)^{\S}$ & 0/107 (0) & $170 / 427(40)$ & $69^{\ddagger} / 252(27)$ \\
\hline Median interval from diagnosis to 3-month in-hospital death in days & - & $7[2 ; 19]$ & $11[5 ; 24]$ \\
\hline Post hospitalization follow-up - no· / no evaluated (\%) & $104 / 107(97)$ & $256 / 257(99)$ & $181 / 181(100)$ \\
\hline Median post hospitalization follow-up in months & $5[3 ; 9]$ & $5[3 ; 11]$ & $5[3 ; 13]$ \\
\hline 3-month post hospitalization death - no $\cdot /$ no $\cdot$ evaluated (\%) & - & $24 / 257(9)$ & $6 / 181(3)$ \\
\hline Median interval from diagnosis to 3-month post hospitalization death in days & - & $54[24 ; 68]$ & $62[58 ; 68]$ \\
\hline New infection during post-hospitalization period- no / no evaluated (\%) & $0 / 104(0)$ & $19 / 255(7)$ & $7 / 181(4)$ \\
\hline Recurrence of listeriosis $\|^{\|}$ & $0 / 104(0)$ & $2 / 255(1)$ & $1 / 179(1)$ \\
\hline
\end{tabular}


Table 3

3-month death in bacteremias + neurolisteriosis $(\mathrm{N}=679)^{*}$

\section{Factors}

Female sex

Age - year

At least one immunosuppressing comorbidity

Ongoing organ neoplasia

Recent weight loss $>5 \mathrm{kgs}$

Intensive care unit management

Multi-organ failure

Aggravation of any pre existing organ dysfunction

Diarrhea

Flu-like symptoms

Monocytopenia $<200 / \mathrm{mm}^{3}$

Neutrophils - cells $/ \mathrm{mm}^{3}$

Cotrimoxazole therapy

Aminoglycoside therapy

Active betalactam therapy ${ }^{\ddagger} \dagger$

3-month death in neurolisteriosis $(\mathrm{N}=\mathbf{2 5}$

\begin{tabular}{cc} 
Odds ratio (95\% CI) $^{+}$ & p-value \\
$1 \cdot 60(1 \cdot 04-2 \cdot 46)$ & $0 \cdot 034$ \\
$1 \cdot 03(1 \cdot 01-1 \cdot 05)$ & $0 \cdot 001$ \\
$0 \cdot 43(0 \cdot 15-1 \cdot 22)$ & $0 \cdot 113$ \\
$5 \cdot 19(3 \cdot 01-8 \cdot 95)$ & $<0 \cdot 001$ \\
$1 \cdot 74(1 \cdot 05-2 \cdot 87)$ & $0 \cdot 031$ \\
$1 \cdot 48(0 \cdot 90-2 \cdot 41)$ & $0 \cdot 120$ \\
$7 \cdot 98(4 \cdot 32-14 \cdot 72)$ & $<0 \cdot 001$ \\
$4 \cdot 35(2 \cdot 79-6 \cdot 81)$ & $<0 \cdot 001$ \\
$0 \cdot 58(0 \cdot 33-1 \cdot 01)$ & $0 \cdot 053$ \\
$0 \cdot 47(0 \cdot 27-0 \cdot 80)$ & $0 \cdot 006$ \\
$3 \cdot 70(1 \cdot 82-7 \cdot 49)$ & $<0 \cdot 001$ \\
$1 \cdot 05(1 \cdot 01-1 \cdot 08)$ & $0 \cdot 006$ \\
$0 \cdot 49(0 \cdot 26-0 \cdot 92)$ & $0 \cdot 027$ \\
$0 \cdot 60(0 \cdot 38-0 \cdot 94)$ & $0 \cdot 024$ \\
$0 \cdot 10(0 \cdot 04-0 \cdot 26)$ & $<0 \cdot 001$ \\
\hline
\end{tabular}

\section{Factors}

Female sex

Age - years

On-going organ neoplasia

Recent major weight loss

Multi-organ failure

Aggravation of any pre existing organ dysfunction

Flu-like symptoms

Mechanical ventilation

Monocytopenia $<200 / \mathrm{mm}^{3}$

Positive blood cultures

Protein concentration in the cerebrospinal fluid

Adjunctive

Neurological impairment in neurolisteriosis $(\mathrm{N}=181)$

Odds ratio $(95 \% \mathrm{CI})^{\dagger} \quad$-value

\begin{tabular}{cc}
$2 \cdot 68(1 \cdot 24-5 \cdot 83)$ & $0 \cdot 013$ \\
$1 \cdot 35(0 \cdot 99-1 \cdot 85)$ & $0 \cdot 058$ \\
$4 \cdot 58(1 \cdot 53-13 \cdot 73)$ & $0 \cdot 007$ \\
$2 \cdot 65(1 \cdot 08-6 \cdot 55)$ & $0 \cdot 034$ \\
$3 \cdot 08(1 \cdot 25-7 \cdot 58)$ & $0 \cdot 014$ \\
$2 \cdot 75(1 \cdot 23-6 \cdot 16)$ & $0 \cdot 014$ \\
$0 \cdot 47(0 \cdot 20-1 \cdot 12)$ & $0 \cdot 087$ \\
$2 \cdot 89(1 \cdot 31-6 \cdot 37)$ & $0 \cdot 009$ \\
$3 \cdot 57(1 \cdot 24-10 \cdot 23)$ & $0 \cdot 018$ \\
$3 \cdot 67(1 \cdot 60-8 \cdot 40)$ & $0 \cdot 002$ \\
$1 \cdot 18(0 \cdot 9-1 \cdot 41)$ & $0 \cdot 062$ \\
$4 \cdot 58(1 \cdot 50-13 \cdot 98)$ & $0 \cdot 008$ \\
\hline
\end{tabular}

\begin{tabular}{lcc}
\hline & Odds ratio (95\%CI) $^{\dagger}$ & p-value \\
Factors & $0.98(0.96-1 \cdot 00)$ & 0.048 \\
Age - years & $21.65(2 \cdot 58-181 \cdot 59)$ & 0.005 \\
Encephalitis symptoms $\$$ & $1.37(1 \cdot 11-1 \cdot 69)$ & 0.004 \\
Number of neurological symptoms & $1.08(0.98-1 \cdot 20)$ & 0.102 \\
\hline Score on Glasgow Coma Scale & \\
\hline
\end{tabular}




\section{Supplementary Material}

This appendix has been provided by the authors to give readers additional information about their work 


\section{Clinical features and prognostic factors of listeriosis: the MONALISA study}

\section{Online Supplemental Materials}

\section{TABLE OF CONTENTS}

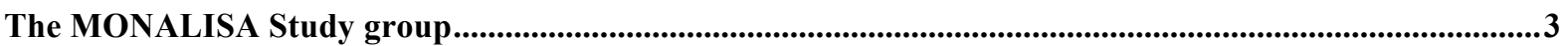

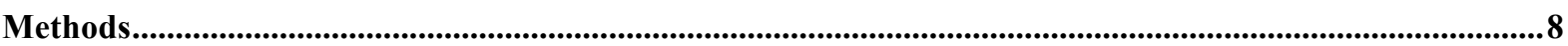

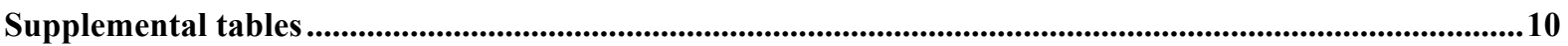

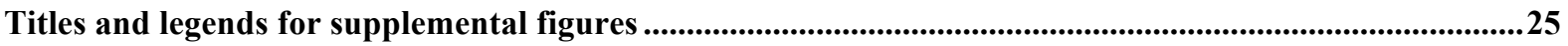

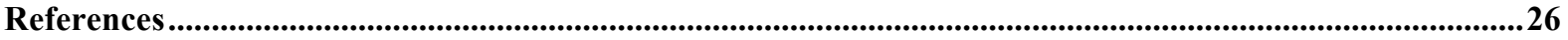

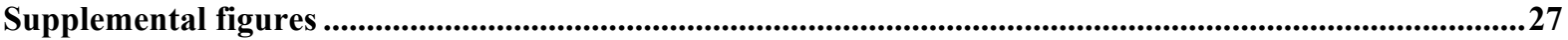




\section{The MONALISA Study group}

Pierre Hausfater, Jean-Louis Pourriat, Enrique Casalino, Bruno Riou, Dominique Pateron, Patrick Yéni, François Bricaire, Yves Ville, Elie Azria, Marc Dommergues, Jean-François Bergmann, Michel Wolff, JeanPaul Mira, Loïc Guillevin, Mathieu Zuber.

Soumeth Abasse, Saïd Aberrane, Pierre Abgueguen, Ayman Abokasem, Bruno Abraham, Chantal AchePapillon, Pascal Adam, Marie-Noëlle Adam, Xavier Adhoute, Daniel Adoue, Moncef Afi, Nawel Afroukh, Ilhem Agha-Mir, Nejla Aissa, Liamine Aissaoui, Grégory Akerman, Ali Akkari, Majed Al Chaar, Faraj Al Freijat, Bachar Al-Jalaby, Didier Albert, Marie-Thérèse Albertini, Hélène Albinet, Gwenaël Alfonsi, Youssef Ali, Zahr-Eddine Ali Chaouche, Anne Allart, Laurent Alric, Alain Améri, Zahir Amoura, Alexandre Ampère, Hakim Amroun, Amévi Ananivi, Pascal Ancelin, Thierry André, Antoine Andremont, Dominique Andreotti, Hélinoro Andriamaneo, Clara Andriau, Hélène Anglaret, Nadia Anguel, Véronique Annaix, Wassila Anteur, Delphine Anuset, Ourida Aoudia, Miloud Arabi, Muriel Archambaud, Maryse Archambaud, Emmanuel Ardiet, Laurent Argaud, Sophie Arista, Guillaume Arlet, Jean Armengaud, Jean-Michel Arnal, Isabelle Arnault, Olivier Arsène, Ziad Assaf, Assi Assi, David Assouline, Dominique Astruc, Yves Aubard, Claude Aubert, JeanPaul Aubry, Marc Auburtin, Philippe Aucher, Philippe Audeguy, Jean-Pierre Audié, Hugues Aumaitre, Michel Aumersier, Jean Auroux, Fanny Autret, Etienne Auvray, Badih Ayach, Paul Aye, Khélifa Ayouz, Elie Azria, Marie-Nadège Bachelier, Thomas Bachelot Philippe Badia, Patrice Badila, Julien Bador, Viorica Badurescu, Olivier Baldesi, Françoise Bandaly, Olivia Bandin, Firouzé Bani-Sadr, Stéphanie Bannier, Thierry Baranger, Isabelle Barazer, Marie-Christine Barbier, François Barbier, Carole Barbier, Marianne Barbieux, Marie, Annick Barboteau, Patricia Barbut, Mylène Baret, François Barière, Jean-Yves Baril, Isabelle Barillot, Geneviève Barjon, Guilène Barnaud, Régine Barraduc, Francis Barraud, Olivier Barraud, Eric Barre, Audrey Barrelet, Jocelyn Barrier, Fréderic Bart, Gérard Barthélémy, Gaëlle Baty, Olivier Baud, Jean-Luc Baudel, Jacques-Olivier Bay, Jérôme Bay, Claude Bazin, Sébastien Beague, Dominique Beal Ardisson, Bertrand Beaune, Anne Beauplet-Lepage, Cécile Bébéar, Dominique Bechade, Céline Becherrawy, Michelle Becker-Schneider, Nathalie Bednarek, Bernard Bedock, Edouard Begon, Zaineb Bekguesmia, Joëlle Belaisch-Amart, Karim Belhadj, Evelyne Belle, Olivier Belmonte, Jean-Pierre Belot, Abdelkader Bemrah, Mouna Ben Soltana, Amélie Benbara, Faiza Benddif-Fin, François Bénézit, Jean-Louis Benifla, Amine Benjelloul, Yacine Benkaci, Abdelaziz Benkhelil, Thierry Benoit-Cattin, Sophie Benoit-Coustou, René-Jean Bensadoun, Thierry Bensaid, Raoul Jacques Bensaude, Henri Bérard, Béatrice Bercot, Bruno Berdin, Marc Berger, Pierre Berger, Smail Bergheul, Jean-François Bergmann, Benoît Bergues, Yvon Berland, Claude Bernard, Louis Bernard, Bérangère Bernardaud, Franck Bernardi, Gilles Bernardin, François Bernasconi, Patrick Bert-Marcaz, Béatrice Berteaux, Anne Berth-Farges, Gilles Berthelot, Jean Berthet, Kevin Bertrand, Anne Bertrou, Marianne Besnard, Emilie Bessede, Philippe Bethery, Claude Beuscart, Odile Beyne-Rauzy, Jean Beytout, Marie-Christine Bezian, Brigitte Bicais, Sabine Bidart, Christian Bidault, Hélène Biessy, Pierre Bigot, Adrianna Bildea, Emmanuelle Bille, Christophe Billy, Nathalie Biron, Jérôme Bizet, Didier Blaise, Agathe Blaise, Gilles Blaison, Mariam Blaka, Pierre Blanc, Stéphane Blanc, Véronique Blanc-Amrane, Pierre Blanchard, Geneviève BlanchardMarche, Michèle Blancs, Caroline Blazejewski, Yves Bléher, Koffi Blewoussi, Romain Blondet, François Blot, Georges-Fabrice Blum, Lucien Bodson, Emilie Boidin, Julien Boileau, Pascal Boileau, Véronique Boin-Gay, Martial Boisseau, Christine Boisselier, Pierre-Edouard Bollaert, Pascal Bolot, Stéphane Bonacorsi, Marie Bonfils, Pascal Bonitchi, Mickael Bonnan, Philippe Bonnefoy, Delphine Bonnet, Richard Bonnet, Emmanuelle Bonnin, Jacques Bor, Marie-Françoise Borie, Bernard Borstein, Philippe Bossi, Yves Botreau, Julie Bottero, Abderrezak Bouasria, Radia Bouaziz, Olivier Bouchaud, Flore Bouche, Gérard Bouchet, Vincent Bouden, Amar Boudhane, Carole-Anne Boudy, François Boue, Cédric Bouet, Jean Claude Boufetteau, Bernard Bouffandeau, Damien Bouhour, Marc Bouiller, Laurence Bouillet, Antoine Bouissou, Luc Boulain, Stéphane Bourgeois, Evelyne Bourgerette, Anne Bourlet, Marc Bourlière, Thomas Bourrée, Aude Bourrouillou, Nicolas Boussekey, Remi Boussier, David Boutoille, Jean-Luc Bouyer, Denise Bouyssou-Destriau, Nicole Bouziges, Michelle Boyer, Louis Braem, Bernard Branger, Catherine Branger, Lucien Brasme, Philippe Bray, Patricia Brazille, Violaine Bresson, Laurent Bret, Anne-Laure Breton, Jacques Breuil, Françoise Brevet, Michel Briaud, Delphine Bridoux, Delphine Briend, Anne-Isabelle Briere, Claire Briere de la Hosseraye, Nathalie Brieu, Jean-Philippe Brieux, Hélène Brihier, Patrick Brisou, Marie-Laure Brival, David Broche, Joëlle Brochen, Camille Bron, Nathalie Bronet, Jacques Bronner, Jean-Pierre Bronowicki, François Brosset, Elisabeth Brottier-Mancini, Sophie Brovedani, Stanislas Bruley des Varannes, Pascale Brunel, Patrick Brunet, Xavier Brunet, Stéphanie Brunet, Maud Brung-Lefebvre, Vincent Brunot, Alain Brusset, Laurence Burc-Struxiano, Michèle Burdin, Thierry Bureau, Fanny Buron, Christophe Burucoa, Jean Cabalion, Severine Cabasson, André Cabié, Victoria Cacheux, Vincent Cadiergue, Guillaume Cadiot, Vincent Caille, Vincent Cailleaux Pierre-Etienne Cailleux, Jocelyne Caillon, Denis Caillot, Mohamed Camara, Emmanuelle Cambau, Gilles Cambonie, Sabine Camiade, Frederique Canis, Meriem Canitrot, Nathalie Canu, Gilles Capellier, Lionel Carbillon, Bernard Carbonelle, Etienne Carbonelle, Marion Carboni, Bruno Carbonne, Francis Carcenac, Florence Cardot, Sylvie Carette, Sylvie Cariou, Pierre Carli, Françoise Carmagnol, François-Xavier Caroli-Bosc, François Caron, Guillaume Cartron, Patrick Casali, Olivier Casanovas, Dominique Cassignard, Denis Castaing, Brisse Castel, Sandrine 
Castelin, Pierre Castelnau, Pascal Cathebras, Jean Catineau, Sylviane Catteu, Blandine Cattier, Christian Cattoen, Vincent Cattoir, Eric Caumes, Jean Caussin, François Cavalié, Franck Ceppa, Charles Cerf, Anne Ceriez, Nathalie Cervantes, Bruno Chabanon-Pouget, Amélie Chabrol, Elisabeth Chachaty, Hanen Chahtour, Fabrice Chaix, Marion Challier, Arnaud Chalvon-Demersay, Guy Chambreuil, Bruno Chaminade, Abdourahim Chamouine, Christine Chandesris Joséphine Chapalain-Cagnon, Alain Chapelle, Chantal Chaplain, Claire Chapuzet, Sylvie Charachon, Christophe Charasse, Thierry Charbonnier, Franck Charlier, Olivier Chassin, Véronique Chassy, Delphine Chatellier, Marlène Chatron, Hélène Chaussade, Pascal Chavanet, Pascale Chavel, Ali Chekroun, Wladimir Chelle, Jonathan Chelly, Christel Cherlet, Alain Chevailler, Pascal Chevalet, Yannick Chevalier, Christian Chidiac, Loïc Chimot, Nabil Chiouk, Philippe Chiron, Catherine Chirouze, Marie-Charlotte Chopin, Gabriel Choukroun, Martine Chouraqui, Kamilla Chraibi, Dominique Chudersky, Michel Chuzeville, Michel Cingotti, Gael Cinquetti, Dragos Ciocan, Isabelle Citony, Catherine Claise, Juliette Clarissou-Philippe, Benoît Claude, Danielle Clave, Christophe Clément, Marie-Thérèse Climas, Daniel Coetmeur, Stéphanie Cognet, Daniel Cohen, Sophie Coignard, Jean-Philippe Coindre, Raphaël Coint, Renato Colamarino, AnneMarie Colingorski, Olivier Collard, Michel Collet, Anne Collignon, Marie-José Collus, Jean-Claude Colombani, Philippe Colombat, Christian Combe, Philippe Condominas, Marie-Chrsitine Conroy, Thierry Constans, Nicole Constantin, Claudine Contamin, Damien Corberand, Anne-Marie Cordier, Charlotte Cordonnier, Hélène Corneloup,Yannick Costa, Frédérique Costa, Mathieu Coste, Corinne Costes, Françoise Cotes, Rabea Cotteret, Louis-Jean Couderc, Pierre Cougoul, Jean-Michel Coulaud, Marie-Pierre Coulhon, Patrice Coulon, René Courcol, Florence Courillon, Johan Courjon, Jérémie Courouble, Dominique Courouge-Dorcier, Henri Courtade, Stéphanie Courtois, Pascal Couturier, Alain Créange, Lise Cremet, Julie Cremniter, Jacques Croize, Valérie Crombe, Ingrid Croquet, Eric Cua, Marie-Noelle Cufi, Patrice Cuvillier, Marie-Françoise Dabysing, Karim Dadoun, Corinne Dagada, Sonia Dahan, Redouane Dahoumane, Jacques Daleas, Dominique Dallay, Benoît Dalle, AlexandreDamage, Lena Damaj, Laurent Damaj Gandhi, Ana Danalaché, Pierre-Eric Danin, Marie-Pierre Danjean, Etienne Danquechin Dorval, Anne Dao, Patrick Daoud, Olivier Darchen, Sylvie Dargère, Bincy Darre, Damien Dassant, Gilles Dassieu, Valérie Dattin-Dorrière, Cedric Daupin, Claire Daurel, JeanClaude Dausset, Mélanie Daval Cote, Gary David, Benjamin Davido, Bertille De Barbeyrac, Michel De Biasi, Hubert De Boysson, Edith De Clareuil, Jean-Michel De Kermadec, Sylvie De Martino, Renaud De Tayrac, Roland De Varax, Sten De Witte, Anne Debernardi, Jean-Luc Deboutin, Marc Debouverie, Christophe Decoene, Anne Decoster, Jean-Winoc Decousser, Dominique Decré, Renaud Defebvre, Nicolas Degand, Jean-Marc Degreff, Michel Deiber, Hélène Delaby, Thierry Delacour, Arnaud Delahaye, Jean-Marie Delarbre, Chantal Delasalle, Claire Delbrouck, Jean-Gilles Delecalle, Sophie Delesalle, Chantal Delesalle, Laurence Delhoustal, Delphine Deligne, Françoise Delisle Mizon, Pierre Delobel, Pierre Delour, Bruno Delpeuch, Marie-Claude Demachy, Martin Demarchi, Marie-Joelle Demarcq, Henri Demontclos, Jean-Christophe Dengo, Sophie Deprecq, Pierre-François Dequin, Olivier Dereeper, Alban Deroux, Aicha Derragui, Julien Desblache, Nicole Desbois-Nogard, Dominique Descamps, Jean-Michel Descamps, Christophe Deschamps, Jacques Deschamps, Franck Desemerie, Luc Desfrere, Joelle Desliers, Laurence Desnoulez, Nicole Desplaces, Nicolas Després, Jean-François Dessin, Marie Destors, Olivier Detante, Mathieu Detave, Laurence Detourmignies, Edouard Devaud, Yves Devaux, Bruno Devaux, François Devianne, Alain Devidas, Philippe Devos, Camille Dewitte, Gisèle Dewulf, Chantal Dhennain, Chloé Di Meglio, Habiboulaye Diallo, Sylvain Diamantis, Baihas Dib, JeanMarc Didier, Anne-Françoise Dillies, Claire Dingremont, Noureddine Djafari, Abdelmajid Djeffal, Hama Djerad, Christophe Dollon, Alexandra Doloy, Yves Domart, Jean-Luc Donay, Loïc Dopeux, Gaelle Dorr, Florence Doucet Populaire, Nathalie Dournon, Michel Dreyfus, Didier Dreyfuss, Damien Du Cheyron, Elodie Dubois, Nicolas Dubois, Nadine Dubosc-Marchenay, Béatrice Dubourdieu, Nadine Dubroca, Jean-Christophe Dubus, Catherine Duche, Francis Duchene, Patrick Dudeffant, Jean-Marie Duez, Pierre Duhaut, Françoise Duluc, Hélène Dumouchel, Bruno Dumoulard, Clarisse Dupin, Michel Dupon, Mathieu Dupont, Damien Dupont, Patrick Dupont, Emmanuelle Dupre-Narlet, Peggy Dupretz, Claire-Antoinette Dupuy, Michel Durand, Annie Durand, Solène Durliat-Ellie, Hélène Durox, Christine Dussopt, Fabien Dutasta, Véronique Duval, Ella Dzeing, Florence Eboue, Cyrille Ede, Hanna Eid, Abderrazak El Yamani, Annie Elbez, Mohamed Eldeghedy, Martine Elena-Daumas, Simon Elhadad, Zoubida Elharie-Heraux, Clarence Eloy, Marie-Etiennette Emeriau, Jean-Philippe Emond, Véronique Equy, Anne-Sophie Erena-Penet, Cecilia Esnault, Laure Esposito, Sandrine Essouri, Laurence Estépa, Vincent Estève, Sabine Etchemendy, Nicolas Ettahar, Marie-Line Eustache, Mathieu Evillard, Françoise Evreux, Jean-Marc Eychene, Didier Eyer, Philippe Eymerit, Jean-Luc Fabre, Claire Fabre, Frédéric Faibis, Lionel Falchero, Odile Falguières, Hassan Fallouh, Nicolas Fanjaud, Bruno Fantin, Ibrahim Farah, Fernanda Farto-Bensasson, Anne-Laure Fauchais, Jean-Pierre Fauchart, Anna Faucher, Jean-Marc Faucheux, Anne Faudon-Gibelin, Pierre Faurie, Claudine Fèbre, Toufik Feddal, Assia Ferhat Carre, Nicole Ferreira-Maident, Milagros Ferreyra, Agnès Ferroni, Tristan Ferry, Pierre Feugier, Jean-Claude Feugier, Hacene Fezoui, Hélène Fiette, Alexandra Fille, Jean-Michel Filloux, Fabien Fily, Marguerite Fines, Cristel Fissore Magdelein, Mathilde Flahault, Christian Floriot, Clovis Foguem, Nathalie Fonsale, Benoît Fontenel, AnneMarie Forest, Anne Forest, Emmanuel Forestier, Louise Fortin, Nicolas Fortineau, Marie-Pierre Fos, Saskia Foucart, Alain-Charles Fouilhoux,, Pierre Fournel, Damien Fournier, François Fourrier, Thibaut Fraisse, Isabelle Fredenucci, Odile Fremin-Batteux, Janine Frey, Luc Frimat, Marie Froidure, Christophe Fruchart, Nathalie Fruleux, Christine Fuhrmann, Michel Fuillet, Nathalie Funakoshi, Alain Fur, Patricia Gabez-Therou, 
Valerie Gaborieau, Sylvie Gabriel-Soléan, Laurence Gachassin, Jean-François Gaide, Julia Gaillard, Jean-Louis Gaillard, Tiphaine Gaillart, Sad Gaizi, Nicolas Gallo, Manuel-Luis Gameiro, Caroline Garandeau, Aurelie Garbi, Yves Garcera, Ghislaine Gardes, Olivier Garesslin, Fabien Garnier, Marie Garofano, Olivier Garosi, Michel Garre, Hélène Garrec, Bernard Garrigues, Anne Gaschet, Alexandre Gascon, Emmanuel Gascou, Jacques Gasnault, Marc Gatfosse, Jean-François Gattault, Hélène Gatti, Bernard Gauche, Philippe Gaudard, Joel Gaudelus, Stéphane Gaudry, Valérie Gauduchon, Jean-Baptiste Gaulthier, Yvan Gauthier, Rémi Gebeile, Loïc Geffray, Françoise Geffroy, Alain Geissler, Maud Gelez, Vincent Gendrin, Guy Gengembre, Hervé Gentilhomme, Sandrine Gérart Pons,Marie-Claude Germain, Julien Gesquière, Adel Ghedira, Christine Ghevaert, Jean-Michel Gillot, Edouard Girard, Serge Girard, Pierre-Marie Girard, Isabelle Girard-Buttaz, Martine Giraud, Isabelle Glorieux, Chandrah Goburdhun, Michel Godin, Catherine Godon, François Goehringer, Sylvie Goffart, Isabelle Goidin, Daniele Goldgran Toledano, François Golfier, Armand Goll, Olivier Gontieron, Anne Goquelin, Laurence Got, Alain Goudeau, Cécile Goujard, François Goupil, François Gouraud, Alain Gourlaouen, Jérôme Gournay, Julien Goustille, Alain Goux, Benoît Grandclerc, Jean-Didier Grange, Thierry Granger, Françoise Granier, Michèle Granier, Denis ,Grasset, Alain Gravet, Isabelle Grawey, Alix Greder Belan, Jean-Jacques Grelaud, Karine Grenet, Bernard Gressier, Bernadette Grignon, Emmanuel Grimprel, Geneviève Grise, Vincent Grobost, Marine Gross Goupil, Olivier Grossi, Brigitte Gruffat, Anne Gruson, Yves Guénard, Arnaud Guérard, Bruno Guérin, Mathilde Guérin, Claude Guérin, Bernard Guerquin, Dominique Guerrot, Benoît Guéry, Philippe Gueudet, Pierre-Yves Gueugniaud, Isabelle Guichard, François Guichart, Ozel Guiden, Bertrand Guider, Pascale Guillarmé Grossmann, François Guillemot, Pierre Guillet, Christelle Guillet-Caruba, Bernard Guillois, Olivier Guilloy, Thomas Guimard, Karine Guimard, Philippe Guimier, Jérôme Guinard, François Guinet, Olivier Guisset, Karine Guitteaud, Laurent Gutmann, Severine Guyetand, Fabian Haccourt, Jean-Pierre Hacot, Tahar Hadou, Stéphanie Haiat, Corinne Haioun, Mostapha Hajjar, Zakaria Hamitou, Sarah Hammami, Sophie Hamon- Charles, Laurence Hamou-Plotkine, Jean-Luc Hanouz, Samia Harbi, Jean, Robert Harlé, Sophie Haro, Christian Harou, Thierry Harvey, Ghada Hatem, MarieHélène Hausermann, Pierre-Alexandre Hauss, Fabrice Hayoun, Moussa Hecham, Xavier Heches, Nourredine Hedjem, Anne Heidt, Nicolas Heisel, Fréderic Heluwaert, Thierry Helvadjian, Tawfiq Henni, Yanne HenryAndrieu, Delphine Hequet, Patrick Herbecq, Raoul Herbrecht, Romain Hernu, Catherine Herry, Jean-Paul Herry, Serge Herson, Marie-Noelle Heurtaux, Emmanuelle Heusse, Catherine Heyraud-Blanchet, Yoar Hichri, Gilles Hilbert, Marie-Thérèse Hili, Sandrine Hiret, Gilles Hittinger, Anne-Cécile Hochart, Nathalie Hodee, Anne Holstein, Cécile Hombrouk-Alet, Stéphane Hominal, Patrick Honderlick, Stéphanie Honoré Bouakline, Bernard Hory, Pierre Hourdebaigt-Larrusse, Serge Houssaye, Nathalie Houssiaux-Maisonneuve, Abdelkader Hrichi, Didier Hubert, Benoît Huc, Karine Humbert, Dominique Hurel, Stéphanie Husson-Wetzel, Pascal Hutin, Bernard Huttin, Laura Hyerle, Silvia Iacobelli, Serge Ilunga, Guenièvre Imbert, Elias Jabre-Sikias, Jean-Louis Jacob, Romain Jacobs, Dominique Jacomy, Pascal Jacquier, Hervé Jacquier, Marie-Christine Jaffarbandjee, Angéline Jamet, Didier Jan, Guyro Jang, Anne-Christine Jaouen, Henry Jardel, Vincent Jarlier, Luc Jarrige, Bernard Jarrousse, Benoît Jauhlac, Véronique Jault, Thierry Jault, Eric Jaunait, Etienne Javouhey, Rodolphe Jean, Julie Jean, Hélène Jean-Pierre, Eliette Jeanmaire, Katy Jeannot, Hassen Jeddi, Mickael Jego, Selim Jennane, Olivier Join-Lambert, Pascal Joly, Olivier Jonquet, Eric Josien, Fabienne Jouatte, Agnès Jouffret, Noémie Jourde-Chiche, Emilie Jourdes, Philippe Jouvencel, Charlotte Jouzel, Geneviève Julienne, Eric Jullian, Agnès Juven, Philippe Juvin, Michel Kaidomar, Thomas Kaiser, Pierre Kalfon, Georges Kaltenbach, Nassim Kamar, Imad Kansau, Ali Kara, Leila Karaoui, Apollinaire Karirisi, Bernard Karkous, Marie Kassis, Samer Kayal, Marie Keller, Chrystelle Kemenar, Samir Kennouche, Aissa Kerchache, Jean-Marc Kerleau, AnneGaelle Kervegant, Sandrine Khalifa-Thellier, Fethi Radhouane-Khanjari, Hakim Kherouf, Nordine Khodeir, Eric Kibbrecht, An Kim, Jean-Paul Kisterman, Bernard Kittschke, Frédéric Klapczynski, Marie-José Kodzin, Bano Konate, Sylvain Kouaho, Laurence Koulmann, Karim Krechiem, Sarah Kubab, Charles Kubiak, JeanEmmanuel Kurtz, Philippe Labadie, Damien Labarrière, Andrea Labaune-Kiss, Aurélie Labé, Isabelle Labourdette, Philippe Labrousse, Anne-Sophie Labussière, Florence Lachenal, Jérôme Lacroix, Alain Lafeuillade, Marie-Odile Lafforgue, Ingrid Lafon, Jean-Pierre Lagasse, Julien Lagrandeur, Jean-Marc Lalot, Mélissa Lalu, Simone Laluque, Sébastien Lamache, Richard Lamarca, Aurore Lamberet, Thierry Lambert, Fabien Lambiotte, Bouchra Lamia, Ghislaine Lamoine-Gimet, Armelle Lamour, Brigitte Lamy, Patrice Lanba, Nathalie Landgraf, Luc Landraud, Jean-Patrick Laporte, Claire Larible, Fabrice Larrazet, Claire Larroche, Claudine Lasbasses, Ludovic Lassel, Bertrand Lassere, Patrice Laudat, Dominique Lauque, Etienne Laurens, Frédéric Laurent, Raphael Lauretta, Max Laurin, Brigitte Lauzanne, Emmanuelle Lavalard, Isabelle Lavenu, Jean-Philippe Lavigne, François Le Baron, Rozenn Le Berre, Pierre-Yves Le Berruyer, Marc Le Bideau, Cécile Le Boterff, Sylvain Le Chevallier, Alain Le Coustumier, Anne Le Du, Valérie Le Goff, Catherine Le Henaff, Geneviève Le Lay, Gilles Le Maout, Athéna Le Pierres, Patricia Le Pimpec, Marguerite Le Poulain, AnneMarie Le Reste, Ludovic Le Sec, Françoise Le Turdu, Sophie Leautez-Nainville, Eddy Lebas, Véronique Leblond, Marie-Annick Lebreton, Didier Lebreton, Evelyne Lecaillon-Thibon, Sylvie Lechat, Catherine Lechiche, Alain Lecis, Roland Leclercq, Claire Lecomte, Hervé Lécuyer, Sylvie Ledru, Dominique Leduc, Véronique Leflon, Christine Lefort, Hélène Lefranc, Jean-Yves Lefrant, Christophe Legendre, Isabelle Legoff, Laurence Legout, Mathieu Legrand, Annick Legras, Antoine Legros, Virginie Leguen, Franck Leibinger, JeanDaniel Lelièvre, Franck Lellouche, Adrien Lemaignen, Romain Lemarie, Chantal Lemble, Olivier Lemenand, 
Fanny Lemercier, Didier Lemery, Bruno Lemmens, Malcolm Lemyze, Michel Leneveu, Françoise Léonetti, Caroline Léonnet, Sophie Léotard, Didier Lepeletier, Didier Lepelletier, Raphael Lepeule, Quentin Lepiller, Laurence Lequen, Lionel Leroux, Stéphane Leroux, Hélène Leroy, Vincent Leroy, Olivier Leroy, Fabrice Lesage, Mathilde Lescat, Ludovic Lesecq, Aude Lessene, Jean-Marc Lessinger, Claire Letellier, Nicolas Letellier, Vincent Letouzey, Lorraine Letranchant, Marion Levast, Yann Leveneur, Thierry Levent, Marie Levy, Marc Levy, David Leysenne, Benoît Libeau, Ebutu Likose, Suzanne Lima, Gérard Lina, Bertrand Lioger, Benoît Lionnet, Anne-Sophie Lipovac, Vincent Loffeier, Françoise Loison, Aurélien Lorchleac'h, Fabienne Lorge, Jean-François Loriferne, Aurélien Lorléa'ch, Guillaume Louart, Isabelle Loury-Lariviere, Véronique Loustaud, Alain Lozniewski, Roger-Charles Luciani, Luca Luminitan Elena Lupean, Pierre Lureau, MarieFrance Lutz-Murphy, Luwawu Mbimba, Damien M'Bey, Abdallah Maakaroun, Valérie Macci, Fatma Magdoud, Guy Mager, Théophile Magna, Jean-François Magny, Chetaou Mahaza, Isabelle Mahé, Christine Maillard, Jean-Jacques Maillet, François Maillet, Sébastien Maillez, François Maillot, Antoine Maisonneuve, Solène Makdessi, Claire Malbrunot, Brigitte Malbruny, Caroline Malderet, Jean-Valère Malfuson, Philippe Malherbe, Patrick Malherbe, Sophie Malhiere, Laurent Mandelbrot, Laurent Mandin, Aziza Mandjee, Benjamin Manéglier, Mohammed Mansouria, Elena Marcu, Jean-Bernard Mariette, Sylvie Mariette, Jacques Markarian, Carole Marmouset, Olivier Marpeau, Olivier Marret, Thierry Marsepoil, Isabelle Marterl-Lafay, Benoît Martha, Patrick Marthelet, Xavier Martin, Florence Martin, Pierre Martin, Régine Martin, Christian Martin, Laurent Martin Lefèvre, Olivier Martineau, Olivier Martinet, Valérie Martinez, Martin Martinot, Pascale Martres, Sophie Marty, Charles Marty-Ane, Paul Marzouk, Géraldine Mascade, Florent Masia, Thierry Masseron, Loïc Masson, Philippe Masson, Isabelle Matheron, Slavius Matica, Olivier Matray, Marie-Françoise Mattei, Bertrand Maubert, Jeanne Maugein, Juan-Pablo Maureira, Thierry May, Adrien May, Vincent Maze, Eric Meaudre Desgouttes, Alexandre Mebazaa, Frédéric Mechai, Virginie Medeau, Richard Megbemado, Mona Mehri, Myriam Mein-Bottini, Adrien Melis, Joelle Mellier, Philippe Ménager, Céline Ménard, Lucile Mendes-Martin, Anne-Claude Menguy, Mohamed Menouar, Taoufik Merabet, Paul Mercury, Corinne Meregnani, Philippe Mérigot, Sylvain Mermont, Yacine Merrouche, Pierre-Gilles Merville, Renaud Mesnage, Louis Mesnard, Aurélie Messager, Thierry Messiaen, Jonathan Messika, Patrick Messner Pellenc, Hervé Métenier, Pierre Metton, Christian Meyer, Michel Mialon, Jordi Miatello, Alban Michaud-herbst, Alain Michault, Sophie Micheli, Jocelyn Michon, Jean Baptiste Michot, Severine Mielczarek, Sophie Mignart, Virginie Mignaut, Audrey Migraine Bouvagnet, Fabrice Mihout, Catherine Mille, Olivier Millet, Noel Milpied, Olivier Mimoz, Jacques Minet, Bertrand Minguet, Mohamad Mohty, Marie-Pierre Moiton, Richard Monarchi, Yannick Monceau, Mehran Monchi, Eric Monlun, Jacques Monsegu, Nathalie Montagne, Ambroise Montcriol, Bernard Montmasson, Paramasiven Mootien, Olivier Moquet, Virginie Morando, Anne-Sohie Moreau, Yves Morel, Emmanuel Morelon, Armelle Morin-Fatome, Mathieu Morincomme, Philippe Morlat, Laurent Mosser, Annie Motard-Picheloup, Philippe Mottaz, Claire Moulinoux, Laurence Mouly, Georges Mourad, Jean-Claude Mouries, Hecham Moussa, Audrey Moustache, Jean-François Muir, Valérie Murbach, Yohann N'guyen, JeanClaude N'guyen, Florence N'guyen-Khac, Jean, Marc Nabholtz, Tarik Naceur, Jean Nakhleh, Aline Nare, Xavier Nassif, Frédérique Nathan-Bonnet, Huguette Negrery, Claude Negrier, Dominique Neri, Hakima Nesrine, Catherine Neuwirth, Eugène Ngami, Elisabeth Carole Ngo Bell, Dieudonné Nicobaharaye, Laurent Nicolet, Laurence Nicolet, Marc Nicolino, , Philippe Niel, Karine Nikodijevic, Jean-Marie Nkunzimana, Christian Noel, Alain Noirot, Philippe Noto, Marie-Noelle Noulard, Christophe Ntalu Nkato, Martine Nyunga, Julien Obiols, Patrick Ochocki, Sylvie Odent, Brigitte Olivier, Gérard Oliviero, Basile Ondze, Oléna Orléva, Paul Orode, Henri Osman, Eric Oswald, Paola Otean, Abderkader Ouazir, Christophe Ozanon, Marc Paccalin, MarieChristine Pages, Bénédicte Paindaveine, Béatrice Pangon, Jean-Marie Pannecouck, Jean-Christophe Paquet, Sabrina Parent, Elisabeth Parisi-Duchene, Jérôme Pasche, Thierry Pasdeloup, David Patin, René-Gilles Patrigeon, Isabelle Patry, Jean-Gabriel Paul, Simona Pavel, Michel Pavic, Jean-Michel Pawlotsky, Christopher Payan, Christiane Payen, Yves Péan, Frédérique Péchinot, André Péchinot, Matthieu Pecquet, Agnès Pélaquier, Hervé Peltier, Pascale Penn, Jean-Loup Pennaforte, Didier Perez, Caroline Périsson, Dominique Perotin, Michèle Perouse de Montclos, Sophie Perreve, Christian Perronne, Dominique Perrotin, Valerie Pesque, Martine Pestel-Caron, Vincent Pestre, Fabrice Petassou, Marie-Cécile Petit, Camille Petit Hoang, Philippe Petitjean, François Pettinelli, Anne-Sophie Peultier,Jean-Marie Piala, Gilles Pialoux, Caroline Piau, Aurélia Picard, Jean-Charles Picaud, Eric Pichard, Martin Pierre, Fabrice Pierre, Paul Pierrot, Emilie Piet, Marie-Claude Piffaut, Bertrand Pigeon, Carole Pignon, Georges Pinon, Jacques Piquet, Vincent Piriou, Eric Placidi, Gillles Plainfosse, Christophe Plane, Isabelle Plantier, Nicolai Claudiu-Plesa, Patrick Plésiat, Patrick Plessis, Chloé Plouzeau-Jayle, Marie-Cécile Ploy, Gilbert Pochmalicki, Joanna Pofelski, Yves Poinsignon, Anne-Sophie Poirier, Françoise Poitevin, Christian Pommier, Bénédicte Ponceau, Christophe Poncelet, Daniela Pop, Martine Porcheron, Réginald Pordes, Eric Porthault, Marc Portneuf, Anne Pottier, Pascal Pouedras, Jocelyne Poulain, Gérard Poulain, Hélène Poupet, Julien Pouyanne, Marie Pouzoullic, Claire Poyart, Bruno Pozzetto, Thierry Prazuck, Dominique Pressac, Fabrice Prévost, Sylvie Prince, Pascal Priollet, Olivier Proost, Daniel Protar, Eric Pujade Lauraine, Antoine Pujol, Louis Puybasset, Jean Puyhardy, Vincent Quentin, Denis Quinsat, Valérie Rabier, Fouzia Radaoui, Florian Radenac, Sylvie Radenne, Didier Raffenot, François Raffi, Hassène Rahmani, Céline Ramanantsoa, Guillaume Ranchon, Dana Ranta, Didier Raoult, Jean-Philippe Rasigade, Emmanuel Rassiat, Olivia Raulin, Alain Ravaud, Nathalie Ravet, Hasinrina Razafimahefa, Mirana Razafimahery, Daniel 
Re, Philippe Real, Paul Rebattu, Catherine Rebeyrotte, Pascal Reboul, Anne-Hélène Reboux, Christine Recule, Jean-Philippe Redonnet, Alexis Redor, Yves Regouby, Claude Rémy, Gisèle Renard, Benoît Renard, Frédéric Renou, Philippe Repellin, Jean-Claude Reveil, Valérie Revel, Anne Reverseau, Mathieu Revest, Philippe Rey, Hyacine Rey, Jany Rey Zermati, Guillermo Reyes Ortega, Alain Reynaud, Jacques Reynes, Nasseur Rezgui, Jacques Riahi, David Ribes, Arnaud Ribier, Christian Richard, Florence Richardin, Agnès Riche, Pascal Richette, Khalid Ridah, Philippe Riegel, Frédéric Riehl, Françoise Rigaux, Christophe Rioux, Gilles Rival, Brigitte Rivière, Henri Robert, René Robert, Pierre-Yves Robillard, Mélanie Roblin, Pascal Roblot, France Roblot, Olivier Rogeaux, Pierre-Marie Roger, Dominique Rohmer-Heitz, Christophe Rolland, Mariam RoncatoSaberane, Isabelle Ronda, Philippe Rondepierre, Anne-Marie Roque-Afonso, David Rosay, Christian Rose, Jean-Baptiste Roseau, Sophie Rosello, Sylvène Rosselli, Serge Rossignol, Lionel Rostaing, Christian Roth, Marie-Rose Rothe, Xavier Roubert, Sébastien Rouget, Ahmed Rouidi, Philippe Roulier, Yannick Rouquet, Isabelle Rouquette-Vincent, Chantal Roure Sobas, Sylvaine Rousseau, FlorenceRousseau, Etienne Roussel, Jean-Christian Roussel, Christophe Roussel, Patricia Roussellier, Christophe Roy, Daniel Royer, JeanChristophe Roze, Bertrand Rozec, Raymond Ruimy, Etienne Ruppé, Olivier Ruyer, Sébastien Sabbat, Tahar Saghi, Christophe Saigne, Necera Sakek, Gilles Salama, Celia Salanoubat, Guenaelle Salaun-Beretta, Elie Saliba, Gilles Salles, Odile Salmon, Jean Louis Salomon, Jean-Michel Salord, Marina Salvucci, Thierry Samson, Richard Sanchez, Dominique Sansot, Mihaela Saplacan, Carlo Saroufim, Philippe Sauder, Denis Sautereau, Ferdinand Savare, Rim Savatier, Anne Scemla, Adeline Schendel, Anna Schmitt, François Schmitt, Alain Schmitt, Morgane Schneerson, Francis Schneider, Jean-François Schved, Carole Schwebel, Anthony Sebban, Aline Secher, Nicholas Sédillot, Yacine Sedjelmaci, Christine Segonds, Camille Seignovert, Corinne Seknazi, Marius Semenescu, Arnaud Sément, Jean-Claude Semet, Guy Semet, Eric Sennevile, Miloud Serier, Arnaud Serre, Nathalie Seta, Jacques-Arnaud Seyrig, Eric Sgro, Joyce Sibony-Prat, Jean-Luc Sicsic, Farid Sifaoui, Thomas Signouret, Nicolas Sigur, Souad Silhadi, Umberto Siméoni, Bernard Simian, Thomas Similowski, Georges Simon, François Simon, Catherine Simonin, Cristina Sirbu, Jean-Marie Sire, Valérie Sivadon-Tardy, Bruno Sivery, Michel Slama, Salim Smati, Rodolphe Sobesky, Hélène Sokeng-Affoule, JeanPierre Sollet, André Sommabère, Daniel Sondag, Florence Soraudeau, Albert Sotto, Bruno Soulie, Laurent Souply, Jean-Christophe Souquet, Pierre Soury, Bertrand Souweine, Jean-Paul Stahl, Marie-Isabelle Steibach, Robin Stephan, Annabelle Stoclin, Philippe Stolidi, Paul Strock, Diana Suatean, Jean-François Subra, Yves Sucin, Philippe Suel, Marc Sullice, Tali-Anne Szwebel, Laurent Tacchini, Hana Talabani-Boizot, Jean-Philippe Talarmin, Fethi Taleb, Catherine Tamalet, Fabienne Tamion, Fabienne Tamion, Olivier Tandonnet, Jacques Tankovic, Véronique Tardy, Christelle Tassot, Gregory Taurin, Sabine Taylor, Xavier Tchenio, Jacques Tebib, Laurent Teillet, Antonio Teixeira, Mustapha Terki, Sonia Tesseydre, Jean-Claude Texier, Anthony Texier, JeanPaul Thellier, Didier Thibaud, Michel Thibault, Freddy Thibaut, Jacques Thierry, Sylvie Thoinet, Pierre Thomas, René Thomas, Yves Thomas, Caroline Thomas, Jean Thore, Jean-Marc Thouret, Maxime Thouvenin, Francis Thuet, Ahmed Tigaizin, Sylvestre Tigaud, Catherine Tiry, Pierre Tissieres, Alain Tissot, Anne Tixier, Kamel Touati, Marie-Lina Toubia, Olivier Toullalan, Jean-Marc Tourani, Bernadette Tourrand, Yvan Touze, Asma Trabelsi-Jnifen, Laurent Traissac, Tu Anh Tran, Vanessa Tran, Joelle Tricoire, Laurent Tronchon, Sébastien Trouiller, David Trystram, Ahmed, Tsouria Becaid, Mirela Tuca, Mathilde Turpin, Bernard Vache, Anne Vachée, Christine Vaillant, Patrick Valadier, Patrick Valayer, Xavier Valette, Françis Vallet, Florence Van de Velde, Serge Vanden Einjden, François Vandenesch, Philippe Vande-perre, Marie-Christine Varin, Gwenaelle Vary, Yvan Vaschalde, Matteo Vassallo, Marc Vasse, Manica Vasseur, Rémi Vatan, JacquesVaucel, Michel Vergnaud, Annick Verhaeghe, Annie Vermesch-Langlin, Guy Vernet, Véronique Vernet Garnier, Virginie Verrier, Annie Vessieres, Véronique Vialette, Jean-Paul Viard, Antoine Vieillard Baron, Alain Vighetto, Marc Villemain, Daniel Villers, Jean-Pierre Vilque, Agnès Vinay, Didier Vincent, Pascal Vincent, Colette Vincent, Simon Vincent, Didier Viole, Jérémie Violette, Gerard Viquesnel, Anne-Laure Virlouvet, Séverine Visentin, Daniel Vittecoq, Nicolas Vodovar, Laurence Vrigneaud, Hoang Vu-Thien, Fanny Vuotto, Estelle Wafo, Michel Wagner, Nicola Walid, Elisabeth Walter, Sarah Watson, Pierre Weinbreck, Eva Weinbronn, Emmanuel Weiss, Thierry Weitten, Yves Welker, Pierre-François Westeel, Olivier Wink, Claire Wintenberger, Paul-Louis Woerther, Michel Wolff, Isabelle Worcel, Anne Wuillai, Marc Wurmser, Jacob Xavier, Yazdan Yazdanpanah, Aihem Yehia, Sterenn Yvenou, Elie Zagdoun, Dominique Zagozda, ThierryPascal Zame, Oana Zamfiri, Hélène Zanaldi, Marc Zandecki, Eric Zaoui, Virginie Zarrouk, Jean-Pierre Zarski, Patrick Zavadil, Valérie Zeller, Franck Zerbib, Vincent Zerr, Lémia Zgarni, Fabien Zoulim, Patrick Zoveda, Pierre Zuber and Christian Zumbo. 


\section{Methods}

\section{Definitions}

Fetal losses included spontaneous abortion and stillbirths Neonatal infections diagnosed $\geq 7$ days of life were classified as late-onset listeriosis. The diagnosis of early onset infection was made when $\mathrm{Lm}$ was identified in CSF or blood cultures, or when $L m$ was identified in any other neonatal sample with concomitant C-reactive protein level $>20 \mathrm{mg} / \mathrm{L}$ at birth. Neonates were considered healthy if they fulfilled the following criteria: normal physical examination, C-reactive protein level $<5 \mathrm{mg} / \mathrm{L}$, CSF nucleated cells count $\leq 30 / \mathrm{mm}^{3}$ when a lumbar puncture was performed and all bacteriological samples tested negative.

Meningeal involvement was defined clinically (nuchal rigidity evocative of listeriosis for the clinician) or biologically (CSF nucleated cells count $>4 / \mathrm{mm}^{3}$ and/or CSF protein $>0.5 \mathrm{~g} / \mathrm{L}$ and/or evidence of $L m$ by culture or quantitative polymerase chain reaction in the CSF). Encephalitis was defined by the presence of at least one of the following symptoms, with no alternative cause than listeriosis identified: altered consciousness (score on Glasgow Coma Scale <15), seizures, new onset of neurological symptoms and abnormality on electroencephalography consistent with encephalitis according to the International Encephalitis Consortium. ${ }^{1}$ Brainstem involvement was defined by cranial nerve palsy involvement (except the olfactive nerve). Patients with neurolisteriosis and positive blood cultures, hence patients with both neurolisteriosis and bacteremia, were classified as patients with neurolisteriosis.

Comorbidities were defined as any significant past or concomitant disease or condition reported at listeriosis onset. To better categorize patients' conditions and distinguish those with possible increased risk of infection, we listed among them those possibly associated with immunosuppression. They were labeled "immunosuppressive comorbidities" and defined as follow: congenital immune deficiency, hematopoietic stem cell transplantation, solid organ transplantation, hematological malignancy, preexisting lymphopenia, preexisting neutropenia, inflammatory rheumatic disorders, inflammatory bowel diseases, other auto-immune diseases, asplenia, HIV infection, prescription of corticosteroids or other immunosuppressive therapies in the past five years, solid organ cancer, diabetes mellitus, cirrhosis, alcohol uptake $>3$ drinks/day, end-stage renal disease. ${ }^{2}$ In addition, age $>70$ years was also retained as an immunosuppressive condition, because of the immunological impairments occur after the age of 70 years. ${ }^{2}$

Aggravation of any pre existing organ dysfunction was defined as any aggravation of preexisting liver, kidney, lung, heart, or pancreas (diabetes) dysfunction at the moment of infection onset.

Multi-organ failure was defined according to the international definition. ${ }^{3}$

Anti-Listeria betalactam included penicillin G, amoxicillin, ticarcillin, piperacillin, imipenem and meropenem.

\section{Data collection and management}

Baseline data at admission were indeed collected at the moment of inclusion. Subsequent in-hospital follow-up and post hospitalization follow-up were also prospectively collected at the end of hospitalization and at least 3 months after diagnosis.

Data presented are those reported by clinicians in charge of the patients Inconsistencies and missing data were checked and corrected and/or completed with clinicians of the 388 centers that included the 818 patients.

All human bio-resources were processed at the ICAReB biobanking platform, Institut Pasteur (NF S96-900, Biobanking and Biomolecular Resources Research Infrastructure participating member (BB-0033-00062)).

\section{Statistical analysis}

The study followed the STROBE reporting guidelines for observational studies. ${ }^{4}$ The sample size was a convenient sample, determined by the number of eligible cases during the study period. This sample size gives a power of $80 \%$ to detect a Cohen's effect size of 022 between neurolisteriosis and bacteremia cases $(\mathrm{n}=252$ and 427 respectively). ${ }^{5}$ Categorical variables were described with frequencies and percentages Quantitative variables were described with the mean and standard deviation (SD) or median and interquartile range $\left[25^{\text {th }}-75^{\text {th }}\right.$ percentile]. To identify differences between patients and between clinical forms of listeriosis we used parametric and non-parametric tests; using $\mathrm{Chi}^{2}$ or Fisher's exact tests to compare categorical variables and Student's t-test or Mann-Whitney tests to compare categorical variables. Fisher's exact tests were used whenever expected counts were below 5 for at least one category and Mann-Whitney tests were used in case of asymmetrical behavior.

We investigated the relation between unfavorable outcome and potential predictors by performing a multivariable logistic analysis. Factors tested for univariable analysis were chosen a priori based on available 
data and pathophysiological relevance. Unfavorable outcome was defined as fetal loss for maternal-neonatal infections, three-month mortality for bacteremia and neurolisteriosis and also persisting neurological impairment for neurolisteriosis. In a first step, variables showing associations at a significance level of $\alpha=0 \cdot 20$ in a univariable analysis were selected for inclusion in the multivariable model. Missing values were then imputed using a Multivariate Imputation by Chained Equations procedure generating 50 imputed data sets assuming data were missing at random. A stepwise selection was performed on the stacked data set with a fixed weight applied to all individuals $(\mathrm{w}=(1-\mathrm{f}) / \mathrm{m}$, where $\mathrm{f}$ is the average fraction of missing data across all variables and $\mathrm{m}$ is the number of imputed data sets). The final multivariable logistic model was applied to each of the 50 imputed data sets and OR and their 95 percent confidence intervals were estimated by using Rubin's rules. ${ }^{6}$ To further characterize cases associated with bacteremia and neurolisteriosis, we performed a hierarchical clustering on principal components. Variables used for this classification were socio-demographics data, medical history, past treatments, clinical and microbiological data. As these variables are not all continuous, we first performed a multiple correspondence analysis (MCA) and then used the coordinates of the individuals on the principal components for the classification. MCA is used to transform categorical variables into continuous ones. We categorized the continuous variables so that they could be introduced into the MCA, according to clinicallyrelevant cut-offs. To ensure stability of the partition, we kept axes of the MCA capturing $90 \%$ of the information, the last axes being considered as noise. We performed a k-means consolidation. This analysis was conducted by using the package FactoMineR. ${ }^{7}$ Missing data for active variables were handled using the package missMDA. ${ }^{8}$

Statistical analysis was conducted with R statistical software (version 322). ${ }^{9}$ All statistical tests were two-tailed and $p$-values $<0.05$ were considered statistically significant. 


\section{Supplemental tables}

\section{Table S1}

\begin{tabular}{|c|c|c|c|c|c|c|c|c|c|}
\hline \multirow{2}{*}{ Characteristic } & \multicolumn{3}{|c|}{ Maternal-neonatal } & \multicolumn{3}{|c|}{ Bacteremia } & \multicolumn{3}{|c|}{ Neurolisteriosis } \\
\hline & Included & Not included & $\mathrm{p}$-value & Included & Not included & $p$-value & Included & Not included & $p$-value \\
\hline $\mathrm{N}=$ & $\mathrm{N}=107$ & $\mathrm{~N}=29$ & & $\mathrm{~N}=427$ & $\mathrm{~N}=173$ & & $\mathrm{~N}=252$ & $\mathrm{~N}=32$ & \\
\hline Age - years & $30 \pm 5$ & $32 \pm 7$ & $0 \cdot 38+$ & $73 \pm 14$ & $76 \pm 15$ & $0.03+$ & $67 \pm 16$ & $76 \pm 14 \ddagger$ & $0.02+$ \\
\hline Male gender - no (\%) & $0 / 107(0)$ & $0 / 29(0)$ & & $246 / 427(58)$ & $108 / 173(62)$ & $0 \cdot 27 t$ & $152(60)$ & $16(50)$ & $0 \cdot 26+$ \\
\hline Death - no / no evaluated (\%)* & $0 / 107(0)$ & $0 / 29(0)$ & & $194 / 427(45)$ & $54 / 130(42)$ & $0.43 \S$ & $75 / 252(30)$ & $10 / 18(56)$ & $0 \cdot 02 \S$ \\
\hline Feta loss- no / no evaluated (\%) & $26 / 107(24)$ & $8 / 19(42)$ & $0 \cdot 1 \S$ & - & - & & - & - & \\
\hline
\end{tabular}

\section{Table S2}

\begin{tabular}{|c|c|c|c|c|c|c|c|}
\hline \multirow[t]{2}{*}{ Pregnancy outcome - no / no evaluated (\%)* } & \multirow[t]{2}{*}{$\begin{array}{l}\text { Mothers } \\
(\mathrm{N}=107)\end{array}$} & \multicolumn{6}{|c|}{ Term at presentation (weeks of gestation) } \\
\hline & & $\begin{array}{l}<25 \\
\mathrm{~N}=25\end{array}$ & $\begin{array}{c}{[25-28[} \\
\mathrm{N}=6\end{array}$ & $\begin{array}{c}{[28-33[} \\
\mathrm{N}=26\end{array}$ & $\begin{array}{c}{[33-37[} \\
N=24\end{array}$ & $\begin{array}{c}{[37-41[} \\
\mathrm{N}=20\end{array}$ & $\begin{array}{l}\text { Late-onset listeriosis } \\
\qquad \mathrm{N}=6\end{array}$ \\
\hline Normal pregnancy/delivery & $5 / 107(5)$ & $3 / 25(12)$ & $0 / 6(0)$ & $1 / 26(4)$ & $1 / 24(4)$ & $0 / 20(0)$ & - \\
\hline Fetal loss & $26 / 107(24)$ & $21 / 25(84)$ & $3 / 6(50)$ & 2/26(8) & $0 / 24(0)$ & $0 / 20(0)$ & - \\
\hline Premature delivery & $48 / 107(45)$ & $1 / 25(4)$ & $3 / 6(50)$ & $23 / 26(88)$ & $21 / 24(88)$ & $0 / 20(0)$ & - \\
\hline Abnormal delivery at term $\dagger$ & $22 / 107(21)$ & $0 / 25(0)$ & $0 / 6(0)$ & $0 / 26(0)$ & $2 / 24(8)$ & $20 / 20(100)$ & - \\
\hline Late-onset neonatal listeriosis & $6 / 107(6)$ & - & - & - & - & - & $6 / 6(100)$ \\
\hline \multirow[t]{2}{*}{ Neonatal outcome for alive infants ${ }^{\ddagger}$} & $\begin{array}{c}\text { Neonates } \\
(\mathrm{N}=82)\end{array}$ & \multicolumn{6}{|c|}{ Term of birth (weeks of gestation) } \\
\hline & & $\begin{array}{c}<25 \\
\mathrm{~N}=0\end{array}$ & $\begin{array}{c}{[25-28[} \\
\mathrm{N}=3\end{array}$ & $\begin{array}{c}{[28-33[} \\
\mathrm{N}=25\end{array}$ & $\begin{array}{c}{[33-37[} \\
\mathrm{N}=21\end{array}$ & $\begin{array}{c}{[37-41[} \\
\mathrm{N}=27\end{array}$ & $\begin{array}{l}\text { Late-onset listeriosis } \\
\qquad \mathrm{N}=6\end{array}$ \\
\hline Intensive care unit management - no / no evaluated (\%) & $40 / 82(49)$ & & $3 / 3(100)$ & $20 / 25(80)$ & $8 / 21(38)$ & $8 / 27(30)$ & $1 / 6(17)$ \\
\hline Median hospitalization duration (days) & $15[8 ; 24]$ & & $32[20 ; 41]$ & $25[20 ; 41]$ & $17[13 ; 21]$ & $7[5 ; 11]$ & $18[8 ; 21]$ \\
\hline In-hospital death - no / no evaluated (\%) & $4 / 82(5)$ & & $1 / 3(33)$ & $3 / 25(12)$ & $0 / 21(100)$ & $0 / 27(100)$ & $0 / 6(0)$ \\
\hline Hydrocephaly reported at 3 months of life - no / no evaluated (\%) & 2/82(2) & & $0 / 3(0)$ & $2 / 25(8)$ & $0 / 21(0)$ & $0 / 27(0)$ & $0 / 6(0)$ \\
\hline Healthy neonate - no / no evaluated $(\%) \S$ & $10 / 82(12)$ & & $0 / 3(0)$ & $1 / 25(4)$ & $0 / 21(0)$ & 9/27 (33) & $0 / 6(0)$ \\
\hline
\end{tabular}


Table S3

\begin{tabular}{|c|c|c|c|}
\hline Characteristics* & $\begin{array}{c}\text { Maternal } \\
(\mathrm{N}=107)\end{array}$ & $\begin{array}{c}\text { Bacteremia } \\
(\mathrm{N}=427)\end{array}$ & Neurolisteriosis $(\mathrm{N}=\mathbf{2 5 2})$ \\
\hline \multicolumn{4}{|l|}{ Non-immunosuppressive comorbidity } \\
\hline Chronic respiratory disease & $2 / 107(2)$ & $77 / 427(18)$ & $32 / 252(13)$ \\
\hline Chronic heart disease & $0 / 107(0)$ & $102 / 427(24)$ & $20 / 252(8)$ \\
\hline Other cardiopathy & $0 / 107(0)$ & $157 / 426(37)$ & $60 / 252(24)$ \\
\hline Hypertension & $0 / 107(0)$ & $188 / 427(44)$ & $110 / 252(44)$ \\
\hline Renal insufficiency & $0 / 107(0)$ & $109 / 427(26)$ & $27 / 252(11)$ \\
\hline Chronic liver disease $^{\dagger}$ & $0 / 107(0)$ & $67 / 427(16)$ & $34 / 252(14)$ \\
\hline Foreign material or implant ${ }^{\ddagger}$ & $2 / 107(2)$ & $153 / 427(36)$ & $54 / 252(21)$ \\
\hline Atopy & $7 / 104(7)$ & $29 / 426(7)$ & $14 / 249(6)$ \\
\hline Psychiatric disorder & $0 / 107(0)$ & $30 / 427(7)$ & $14 / 252(6)$ \\
\hline Recent major weight loss & $0 / 106(0)$ & $115 / 424(27)$ & $45 / 249(18)$ \\
\hline \multicolumn{4}{|l|}{ Immunosuppressive comorbidity } \\
\hline Daily alcohol uptake $>3$ glasses / day & $1 / 107(1)$ & $20 / 421(5)$ & $32 / 246(13)$ \\
\hline Cirrhosis & $0 / 107(0)$ & $49 / 427(12)$ & $20 / 251(8)$ \\
\hline Diabetes mellitus & $0 / 107(0)$ & $94 / 427(22)$ & $55 / 252(22)$ \\
\hline End stage renal disease & $0 / 107(0)$ & $33 / 427(8)$ & $4 / 252(2)$ \\
\hline Solid organ cancer & $0 / 107(0)$ & $160 / 427(38)$ & $49 / 252(19)$ \\
\hline Hematological malignancy & $0 / 107(0)$ & $59 / 427(14)$ & $34 / 252(14)$ \\
\hline Hematopoietic stem cell transplantation & $0 / 107(0)$ & $11 / 427(3)$ & $0 / 252(0)$ \\
\hline Solid organ transplantation ${ }^{\S}$ & $0 / 107(0)$ & $14 / 427(3)$ & $5 / 252(2)$ \\
\hline Asplenia & $0 / 107(0)$ & $7 / 427(2)$ & $3 / 252(1)$ \\
\hline Pre-existing neutropenia (neutrophils count $<500 / \mathrm{mm}^{3}$ ) & $0 / 107(0)$ & $24 / 427(6)$ & $8 / 252(3)$ \\
\hline Pre-existing lymphopenia (lymphocytes count $<1000 / \mathrm{mm}^{3}$ ) & $0 / 107(0)$ & $81 / 426(19)$ & $27 / 252(11)$ \\
\hline HIV infection & $2 / 107(2)$ & $4 / 427(1)$ & $4 / 252(2)$ \\
\hline Inflammatory bowel diseases ${ }^{\|}$ & $1 / 107(1)$ & $9 / 427(2)$ & $12 / 252(5)$ \\
\hline Inflammatory rheumatic disorders** & $2 / 107(2)$ & $26 / 427(6)$ & $20 / 252(8)$ \\
\hline Other auto-immune diseases & $2 / 107(2)$ & $28 / 427(7)$ & $11 / 252(4)$ \\
\hline Congenital immune deficiency & $0 / 107(0)$ & $1 / 427(1)$ & $0 / 252(0)$ \\
\hline Age $>70$ years & $0 / 107(0)$ & $266 / 427(62)$ & $117 / 252(46)$ \\
\hline \multicolumn{4}{|l|}{ Administration of therapies in the past 5 years } \\
\hline Corticosteroids & $1 / 107(1)$ & $146 / 427(34)$ & $79 / 252(31)$ \\
\hline Anti-TNF biotherapy & $1 / 107(1)$ & $5 / 427(1)$ & $6 / 252(2)$ \\
\hline Other immunosuppressive therapy & $1 / 107(1)$ & $137 / 427(32)$ & $71 / 252(28)$ \\
\hline Any corticosteroid/immunosuppressive therapy & $2 / 107(2)$ & $190 / 427(45)$ & $103 / 252(40)$ \\
\hline \multicolumn{4}{|l|}{ Administration of drugs at the moment of listeriosis } \\
\hline Corticosteroids & $1 / 107(1)$ & $103 / 425(24)$ & 48/251 (19) \\
\hline Anti-TNF biotherapy & $0 / 107(0)$ & $1 / 427(1)$ & $6 / 252(2)$ \\
\hline Any corticosteroid/immunosuppressive therapy & 1/107 (1) & $138 / 427(32)$ & $71 / 252(28)$ \\
\hline Administration of anti-acid therapy & $12 / 103(12)$ & $189 / 416(45)$ & $81 / 250(32)$ \\
\hline Administration of prophylactic cotrimoxazole & $0 / 105(0)$ & $14 / 422(3)$ & $7 / 249(3)$ \\
\hline
\end{tabular}




\section{Table S4}

\section{A $\cdot$ Univariable analysis of fetal loss in maternal cases}

\begin{tabular}{|c|c|c|c|}
\hline Characteristics & No, $\mathbf{n}=77$ & Yes, $\mathbf{n}=\mathbf{3 0}$ & p-value \\
\hline \multicolumn{4}{|l|}{ Socio-demographic parameters } \\
\hline Geographic origin & & & $0 \cdot 709 *$ \\
\hline Missing data & $0(0)$ & $0(0)$ & \\
\hline Africa & $26(74 \cdot 3)$ & $9(25 \cdot 7)$ & \\
\hline Else & $51(70 \cdot 8)$ & $21(29 \cdot 2)$ & \\
\hline \multicolumn{4}{|l|}{ Past history } \\
\hline \multicolumn{4}{|l|}{ Past history of fetal loss } \\
\hline weeks of gestation) & & & $0.768 \dagger$ \\
\hline Missing data & $0(0)$ & $0(0)$ & \\
\hline No & $66(72 \cdot 5)$ & $25(27 \cdot 5)$ & \\
\hline Yes & $11(68 \cdot 8)$ & $5(31 \cdot 2)$ & \\
\hline \multicolumn{4}{|l|}{ Clinical / Biological parameters } \\
\hline Maternal fever & & & $0 \cdot 372 *$ \\
\hline Missing data & $0(0)$ & $0(0)$ & \\
\hline No & $19(79 \cdot 2)$ & $5(20 \cdot 8)$ & \\
\hline Yes & $58(69 \cdot 9)$ & $25(30 \cdot 1)$ & \\
\hline Maternal diarrhoea & & & $0 \cdot 684 \dagger$ \\
\hline Missing data & $0(0)$ & $0(0)$ & \\
\hline No & $72(72 \cdot 7)$ & $27(27 \cdot 3)$ & \\
\hline Yes & $5(62 \cdot 5)$ & $3(37 \cdot 5)$ & \\
\hline Maternal flu-like symptoms & & & $0 \cdot 932 *$ \\
\hline Missing data & $0(0)$ & $0(0)$ & \\
\hline No & $52(72 \cdot 2)$ & $20(27 \cdot 8)$ & \\
\hline Yes & $25(71 \cdot 4)$ & $10(28 \cdot 6)$ & \\
\hline \multicolumn{4}{|l|}{ Amniotic fluid macroscopic } \\
\hline aspect & & & $0 \cdot 431^{*}$ \\
\hline Missing data & $0(0)$ & $14(46 \cdot 7)$ & \\
\hline Normal & $35(79 \cdot 5)$ & $9(20 \cdot 5)$ & \\
\hline Stained & $42(85 \cdot 7)$ & $7(14 \cdot 3)$ & \\
\hline Maternal blood cultures & & & $0 \cdot 896^{*}$ \\
\hline Missing data & $16(20 \cdot 8)$ & $6(20)$ & \\
\hline Negative & $27(71 \cdot 1)$ & $11(28 \cdot 9)$ & \\
\hline Positive $(L \cdot$ monocytogenes $)$ & $34(72 \cdot 3)$ & $13(27 \cdot 7)$ & \\
\hline \multicolumn{4}{|l|}{ Term at maternal diagnosis of } \\
\hline listeriosis & & & $<0.001 \dagger$ \\
\hline Missing data & $0(0)$ & $0(0)$ & \\
\hline$[0,25)$ & $4(16)$ & $21(84)$ & \\
\hline$[25,28)$ & $2(33 \cdot 3)$ & $4(66 \cdot 7)$ & \\
\hline$[28,33)$ & $21(80 \cdot 8)$ & $5(19 \cdot 2)$ & \\
\hline$[33,37)$ & $24(100)$ & $0(0)$ & \\
\hline$[37,41]$ & $20(100)$ & $0(0)$ & \\
\hline Post-natal infection & $6(100)$ & $0(0)$ & \\
\hline \multicolumn{4}{|l|}{ Maternal C-reactive protein } \\
\hline level- mg/L & & & $0 \cdot 311+$ \\
\hline Missing data & $14(18 \cdot 2)$ & $2(6 \cdot 7)$ & \\
\hline $\operatorname{Min} / \operatorname{Max}$ & $10 / 331$ & $10 / 321$ & \\
\hline Med [Q1 ; Q3] & $71[37 ; 133]$ & $110[57 \cdot 5 ; 148 \cdot 25]$ & \\
\hline Mean (sd) & $96 \cdot 78(77 \cdot 52)$ & $113(66 \cdot 25)$ & \\
\hline \multicolumn{4}{|l|}{ Maternal blood } \\
\hline $\begin{array}{l}\text { polymorphonuclear cells cour } \\
\text { cells } / \mathrm{mm}^{3}\end{array}$ & & & $0 \cdot 593+$ \\
\hline Missing data & $16(20 \cdot 8)$ & $3(10)$ & \\
\hline $\operatorname{Min} / \operatorname{Max}$ & $1233 / 83000$ & $2213 / 30210$ & \\
\hline Med [Q1 ; Q3] & $9750[5900 ; 14424]$ & $12800[10193 ; 15970]$ & \\
\hline Mean (sd) & $12051 \cdot 33(11094 \cdot 09)$ & $13036 \cdot 56(6033 \cdot 17)$ & \\
\hline \multicolumn{4}{|c|}{ Maternal blood monocytes count- } \\
\hline cells $/ \mathrm{mm}^{3}$ & & & $0 \cdot 606 t$ \\
\hline Missing data & $35(45 \cdot 5)$ & $13(43 \cdot 3)$ & \\
\hline $\operatorname{Min} / \operatorname{Max}$ & $65 / 2056$ & $201 / 1410$ & \\
\hline Med [Q1; Q3] & $690[460 ; 828 \cdot 25]$ & $733[490 ; 990]$ & \\
\hline Mean (sd) & $702 \cdot 79(377 \cdot 95)$ & $758 \cdot 71(371 \cdot 21)$ & \\
\hline \multicolumn{4}{|l|}{ Monocytopenia $<200 / \mathrm{mm}^{3}$} \\
\hline Missing data & $35(45 \cdot 5)$ & $13(43 \cdot 3)$ & \\
\hline$>=200$ & $41(70 \cdot 7)$ & $17(29 \cdot 3)$ & \\
\hline$<200$ & $1(100)$ & $0(0)$ & \\
\hline \multicolumn{4}{|l|}{ Maternal blood lymphocytes } \\
\hline count - cells $/ \mathrm{mm}^{3}$ & & & $0 \cdot 753 \%$ \\
\hline Missing data & $17(22 \cdot 1)$ & $3(10)$ & \\
\hline
\end{tabular}


Min/Max

Med [Q1; Q3]

Mean (sd)

Maternal lymphopenia $(<1500$

$/ \mathrm{mm}^{3}$ )

Missing data

$>=1500$

$<1500$

Clonal complex

Missing data

Other

CC1 24 or 6

Clonal complex

Missing data

Other

CC4

Clonal complex

Missing data

Other

CC6

Urinary tract signs

Missing data

Absent

Present

Treatment

Time interval from first symptom

to treatment

Missing data

No treatment

$<0$ day

$[0 ; 1]$ day

$>1$ day

Time interval from diagnosis to

treatment

Missing data

No treatment

$<0$ day

$[0 ; 3]$ day

$>3$ day

Time interval from admission to

treatment

Missing data

No treatment

$[0 ; 1]$ day

$>1$ day

Time interval from diagnosis to

treatment

Missing data

No treatment

$[0 ; 1]$ day

$>1$ day
$128 / 12000$

$1645[1087 \cdot 5 ; 2306 \cdot 5]$

$1997 \cdot 65(1906 \cdot 16)$

$17(22 \cdot 1)$

$33(64 \cdot 7)$

27 (75)

$0(0)$

$26(72 \cdot 2)$

$51(72 \cdot 9)$

$0(0)$

$60(71 \cdot 4)$

$17(77 \cdot 3)$

$0(0)$

$64(72 \cdot 7)$

$13(72 \cdot 2)$

$0(0)$

$75(74 \cdot 3)$

$2(33 \cdot 3)$

(3)

$0(0)$
$9(90)$
$15(57 \cdot 7)$
$47(77)$
$6(60)$

$0(0)$

$9(90)$

$15(57 \cdot 7)$

$53(77 \cdot 9)$

$0(0)$

$0(0)$

$9(90)$

59 (72)

9 (60)

$5(6 \cdot 5)$

$9(90)$

$43(74 \cdot 1)$

$20(62 \cdot 5)$
0 (0)

1 (10)

$11(42 \cdot 3)$

14 (23)

4 (40)

0 (0)

$1(10)$

$11(42 \cdot 3)$

$15(22 \cdot 1)$

3 (100)

$0 \cdot 307^{*}$

$0 \cdot 945^{*}$

0.584*

$1 †$

$0.051 †$

$0 \cdot 137 \dagger$

$0 \cdot 005$ †

$0(0)$

1 (10)

23 (28)

6 (40)

$2(6 \cdot 7)$

$1(10)$

$15(25 \cdot 9)$

$12(37 \cdot 5)$ 


\begin{tabular}{|c|c|c|c|}
\hline Characteristic & No, $n=410$ & Yes, $n=269$ & p-value \\
\hline \multicolumn{4}{|l|}{ Socio-demographic parameters } \\
\hline Sex & & & $0 \cdot 029 *$ \\
\hline Missing data & $0(0)$ & $0(0)$ & \\
\hline Female & $156(55 \cdot 5)$ & $125(44 \cdot 5)$ & \\
\hline Male & $254(63 \cdot 8)$ & $144(36 \cdot 2)$ & \\
\hline Age at diagnostic & & & $<0 \cdot 001 \ddagger$ \\
\hline Missing data & $0(0)$ & $0(0)$ & \\
\hline $\operatorname{Min} / \operatorname{Max}$ & $0 \cdot 79 / 98 \cdot 81$ & $22 \cdot 78 / 99 \cdot 69$ & \\
\hline Med [Q1 ; Q3] & $71 \cdot 36[60 \cdot 46 ; 79 \cdot 55]$ & $77 \cdot 36[64 \cdot 44 ; 83 \cdot 01]$ & \\
\hline Mean (sd) & $68 \cdot 26(16 \cdot 26)$ & $73.94(12.66)$ & \\
\hline Geographical origin & & & $0.003 t$ \\
\hline Missing data & $3(0 \cdot 7)$ & $5(1 \cdot 9)$ & \\
\hline Africa & $29(78 \cdot 4)$ & $8(21 \cdot 6)$ & \\
\hline Other & $19(76)$ & $6(24)$ & \\
\hline Europe & $32(45 \cdot 7)$ & $38(54 \cdot 3)$ & \\
\hline France & $327(60 \cdot 7)$ & $212(39 \cdot 3)$ & \\
\hline \multicolumn{4}{|l|}{ Past history } \\
\hline At least one comorbidity & & & $0 \cdot 002 *$ \\
\hline Missing data & $0(0)$ & $0(0)$ & \\
\hline No & $30(85 \cdot 7)$ & $5(14 \cdot 3)$ & \\
\hline Yes & $380(59)$ & $264(41)$ & \\
\hline At least one immunosuppressing comorbidity" & & & $<0 \cdot 001^{*}$ \\
\hline Missing data & $0(0)$ & $0(0)$ & \\
\hline No & $41(87 \cdot 2)$ & $6(12 \cdot 8)$ & \\
\hline Yes & $369(58 \cdot 4)$ & $263(41 \cdot 6)$ & \\
\hline Past history of neoplasia or ongoing neoplasia & & & $0^{*}$ \\
\hline Missing data & $0(0)$ & $0(0)$ & \\
\hline No & $322(68 \cdot 5)$ & $148(31 \cdot 5)$ & \\
\hline Yes & $88(42 \cdot 1)$ & $121(57 \cdot 9)$ & \\
\hline Ongoing organ neoplasia & & & $0^{*}$ \\
\hline Missing data & $0(0)$ & $2(0 \cdot 7)$ & \\
\hline No & $366(67 \cdot 7)$ & $175(32 \cdot 3)$ & \\
\hline Yes & $44(32 \cdot 4)$ & $92(67 \cdot 6)$ & \\
\hline Bone marrow transplantation & & & $1+$ \\
\hline Missing data & $0(0)$ & $0(0)$ & \\
\hline No & $403(60 \cdot 3)$ & $265(39 \cdot 7)$ & \\
\hline Yes & $7(63 \cdot 6)$ & $4(36 \cdot 4)$ & \\
\hline Recent weight loss $>5 \mathrm{kgs}$ & & & $<0 \cdot 001 *$ \\
\hline Missing data & $2(0 \cdot 5)$ & $4(1 \cdot 5)$ & \\
\hline No & $332(64 \cdot 7)$ & $181(35 \cdot 3)$ & \\
\hline Yes & $76(47 \cdot 5)$ & $84(52 \cdot 5)$ & \\
\hline Hemodialysis & & & $0 \cdot 050 *$ \\
\hline Missing data & $0(0)$ & $0(0)$ & \\
\hline No & $382(59 \cdot 5)$ & $260(40 \cdot 5)$ & \\
\hline Yes & $28(75 \cdot 7)$ & $9(24 \cdot 3)$ & \\
\hline \multicolumn{4}{|l|}{ Clinical / Biological parameters } \\
\hline Listeriosis form & & & $<0 \cdot 001^{*}$ \\
\hline Missing data & $0(0)$ & $0(0)$ & \\
\hline Neurolisteriosis & $177(70 \cdot 2)$ & $75(29 \cdot 8)$ & \\
\hline Bacteremia & $233(54 \cdot 6)$ & $194(45 \cdot 4)$ & \\
\hline Intensive care unit management & & & $0 \cdot 084^{*}$ \\
\hline Missing data & $0(0)$ & $0(0)$ & \\
\hline No & $275(62 \cdot 8)$ & $163(37 \cdot 2)$ & \\
\hline Yes & $135(56)$ & $106(44)$ & \\
\hline Multiorgan failure & & & $<0 \cdot 001^{*}$ \\
\hline Missing data & $0(0)$ & $0(0)$ & \\
\hline No & $387(69 \cdot 7)$ & $168(30 \cdot 3)$ & \\
\hline Yes & $23(18 \cdot 5)$ & $101(81 \cdot 5)$ & \\
\hline Aggravation of any pre existing organ dysfunction & & & $<0 \cdot 001^{*}$ \\
\hline Missing data & $0(0)$ & $0(0)$ & \\
\hline No & $325(74)$ & $114(26)$ & \\
\hline Yes & $85(35 \cdot 4)$ & $155(64 \cdot 6)$ & \\
\hline
\end{tabular}


Systolic blood pressure $<80 \mathrm{mmHg}$

Missing data

No

Yes

Diastolic blood pressure $<60 \mathrm{mmHg}$

Missing data

No

Yes

Heart rate $>120 / \mathrm{min}$

Missing data

No

Yes

Glasgow coma scale

Missing data

Min/Max

Med [Q1 ; Q3]

Mean (sd)

Diarrhoea

Missing data

No

Yes

Septic shock

Missing data

No

Yes

Flu-like symptoms

Missing data

No

Yes

C-reactive protein level- $\mathrm{mg} / \mathrm{L}$

Missing data

Min/Max

Med [Q1 ; Q3]

Mean (sd)

Blood monocytes count $<200 / \mathrm{mm} 3$

Missing data

No

Yes

Blood PMN cells count - cells $/ \mathrm{mm} 3$

Missing data

Min/Max

Med [Q1 ; Q3]

Mean (sd)

Blood lymphocytes count $<1500 / \mathrm{mm} 3$

Missing data

No

Yes

Clonal complex

Missing data

Other

CC1 24 or 6

\section{Treatment}

Time interval from diagnosis to treatment

Missing data

No treatment

$<0 \mathrm{~d}$

$[0 ; 1] \mathrm{d}$

$>1 \mathrm{~d}$

$15[14 ; 15]$

$13 \cdot 72(2 \cdot 36)$

$81 \cdot 5[39 ; 150]$ $103 \cdot 41(80 \cdot 6)$

$7320[4463 \cdot 5$;

$8297 \cdot 3(5475 \cdot 96)$

Time interval from admission to treatment

Missing data

No treatment

$[0 ; 1] \mathrm{d}$
$93(63 \cdot 1)$

$7(50)$

$10(2 \cdot 4)$

$343(64 \cdot 2)$

$57(56 \cdot 4)$

$23(5 \cdot 6)$

$358(65 \cdot 2)$

$29(45 \cdot 3)$

$6(1 \cdot 5)$

$3 / 15$

$0(0)$

$322(58 \cdot 7)$

$88(67 \cdot 7)$

$0(0)$

$404(60 \cdot 4)$

$6(60)$

$0(0)$

$291(55 \cdot 6)$

$119(76 \cdot 3)$

$26(6 \cdot 3)$

$0 / 360$

$53(12 \cdot 9)$

$326(63 \cdot 3)$

$31(42 \cdot 5)$

$7(1 \cdot 7)$

$100 / 34900$

10770.5]

$9(2 \cdot 2)$

$79(67 \cdot 5)$

$322(59 \cdot 9)$

$2(0 \cdot 5)$

$219(60 \cdot 2)$

$189(61)$

$$
\begin{array}{r}
1(0 \cdot 2) \\
0(0) \\
71(57 \cdot 7)
\end{array}
$$$$
0(0)
$$

$233(62 \cdot 5)$

$105(69 \cdot 5)$

$1(0 \cdot 2)$

$14[12 ; 15]$

$12 \cdot 97(3.09)$

$108[61 ; 188]$

$130 \cdot 55(96 \cdot 83)$

$8700[5370 ; 13511]$

$10047 \cdot 33(7639 \cdot 23)$

$271(64 \cdot 7)$
$32(11 \cdot 9)$

$230(36 \cdot 9)$

7 (50)

$34(12 \cdot 6)$

$191(35 \cdot 8)$

$44(43 \cdot 6)$

$43(16)$

$191(34 \cdot 8)$

$35(54 \cdot 7)$

$9(3 \cdot 3)$

$3 / 15$

$0(0)$

$227(41 \cdot 3)$

$42(32 \cdot 3)$

$0(0)$

$265(39 \cdot 6)$

4 (40)

$0(0)$

$232(44 \cdot 4)$

$37(23 \cdot 7)$

$26(9 \cdot 7)$

$3 / 524$

$38(14 \cdot 1)$

$189(36 \cdot 7)$

$42(57 \cdot 5)$

$16(5 \cdot 9)$

$20 / 67000$

$15(5 \cdot 6)$

$38(32 \cdot 5)$

$216(40 \cdot 1)$

$3(1 \cdot 1)$

$145(39 \cdot 8)$

$121(39)$

$0 \cdot 403+$

$0 \cdot 137^{*}$

0.002 *

$0 \cdot 001 \ddagger$

$0 \cdot 058 *$

$<0 \cdot 001 *$

$<0.001 \ddagger$

$0 \cdot 001 *$

$0 \cdot 002 \div$

$0 \cdot 123^{*}$

$0 \cdot 832$ *

$<0 \cdot 001 *$

$31(100)$

$52(42 \cdot 3)$

$140(37 \cdot 5)$

$46(30 \cdot 5)$

$0(0)$

$31(100)$

$148(35 \cdot 3)$ 
$>1 \mathrm{~d}$

Time interval from first symptom to treatment

Missing data

No treatment

$<0 \mathrm{~d}$

$[0 ; 1] \mathrm{d}$

$>1 \mathrm{~d}$

Duration of bitherapy

Anti-listeria betalactamin ${ }^{* *}+$ aminoglycoside

Missing data

$0 \mathrm{~d}$

$<=3 \mathrm{~d}$

$>3 \mathrm{~d}$

Duration of bitherapy

Cotrimoxazole + aminoglycoside

Missing data

$0 \mathrm{~d}$

$<=3 \mathrm{~d}$

$>3 \mathrm{~d}$

Duration of tritherapy

Anti-listeria betalactam ${ }^{* *}$, cotrimoxazole and aminoglycoside

Missing data

$0 \mathrm{~d}$

$<=3 \mathrm{~d}$

$>3 \mathrm{~d}$

Cotrimoxazole administration

Missing data

No

Yes

Aminoglycoside administration

Missing data

No

Yes

Anti-listeria betalactam administration ${ }^{* *}$

Missing data

No

Yes
$138(60 \cdot 5)$

$11(2 \cdot 7)$

$0(0)$

$5(71 \cdot 4)$

$132(62 \cdot 9)$

$262(69 \cdot 3)$

$0(0)$

$121(45 \cdot 1)$

$76(59 \cdot 8)$

$213(75)$

$0(0)$

$385(59 \cdot 9)$

$16(69 \cdot 6)$

$9(69 \cdot 2)$

$387(59 \cdot 6)$

$15(75)$

8 (80)

$0(0)$

$344(58 \cdot 5)$

$66(72 \cdot 5)$

$0(0)$

$118(46 \cdot 1)$

$292(69)$

$0(0)$

$8(11 \cdot 3)$

$402(66 \cdot 1)$
$90(39 \cdot 5)$

$42(15 \cdot 6)$

31 (100)

$2(28 \cdot 6)$

$78(37 \cdot 1)$

$116(30 \cdot 7)$

$<0 \cdot 001 *$

$147(54 \cdot 9)$

$51(40 \cdot 2)$

$71(25)$

$$
0(0)
$$

$258(40 \cdot 1)$

$7(30 \cdot 4)$

$4(30 \cdot 8)$

$$
0(0)
$$

$262(40 \cdot 4)$

5 (25)

2 (20)

$$
0(0)
$$

$244(41 \cdot 5)$

$25(27 \cdot 5)$

$0(0)$

$138(53 \cdot 9)$

131 (31)

$<0.001 *$

0.011*

$$
0(0)
$$

$63(88 \cdot 7)$

$206(33.9)$ 


\section{Univariable analysis of 3-month death in neurolisteriosis cases}

\begin{tabular}{|c|c|c|c|}
\hline Characteristic & No, $\mathbf{n}=177$ & Yes, $n=75$ & p-value \\
\hline \multicolumn{4}{|l|}{ Socio-demographic parameters } \\
\hline Sex & & & $0 \cdot 079 *$ \\
\hline Missing data & $0(0)$ & $0(0)$ & \\
\hline Female & $64(64)$ & $36(36)$ & \\
\hline Male & $113(74 \cdot 3)$ & $39(25 \cdot 7)$ & \\
\hline Age at diagnostic & & & $<0.001 \dagger$ \\
\hline Missing data & $0(0)$ & $0(0)$ & \\
\hline Min/Max & $0 \cdot 8 / 97 \cdot 1$ & $38 \cdot 5 / 92 \cdot 4$ & \\
\hline Med [Q1; Q3] & $67 \cdot 2[57 \cdot 6 ; 75 \cdot 5]$ & $75 \cdot 9[65 \cdot 1 ; 82 \cdot 1]$ & \\
\hline Mean (sd) & $64 \cdot 2(17 \cdot 4)$ & $72 \cdot 9(12)$ & \\
\hline Geographical origin & & & $0 \cdot 094+$ \\
\hline Missing data & $0(0)$ & $0(0)$ & \\
\hline Africa & $12(80)$ & $3(20)$ & \\
\hline Other & $6(100)$ & $0(0)$ & \\
\hline Europe & $14(53 \cdot 8)$ & $12(46 \cdot 2)$ & \\
\hline France & $145(70 \cdot 7)$ & $60(29 \cdot 3)$ & \\
\hline \multicolumn{4}{|l|}{ Past history } \\
\hline At least one comorbidity & & & $0 \cdot 072 *$ \\
\hline Missing data & $0(0)$ & $0(0)$ & \\
\hline No & $23(85 \cdot 2)$ & $4(14 \cdot 8)$ & \\
\hline Yes & $154(68 \cdot 4)$ & $71(31 \cdot 6)$ & \\
\hline At least one immunosuppressing comorbidity ${ }^{\|}$ & & & $0 \cdot 024 *$ \\
\hline Missing data & $0(0)$ & $0(0)$ & \\
\hline No & $31(86 \cdot 1)$ & $5(13 \cdot 9)$ & \\
\hline Yes & $146(67 \cdot 6)$ & $70(32 \cdot 4)$ & \\
\hline Past history of neoplasia or ongoing neoplasia & & & $<0 \cdot 001^{*}$ \\
\hline Missing data & $0(0)$ & $0(0)$ & \\
\hline No & $154(75 \cdot 9)$ & $49(24 \cdot 1)$ & \\
\hline Yes & $23(46 \cdot 9)$ & $26(53 \cdot 1)$ & \\
\hline Ongoing organ neoplasia & & & $<0.001 *$ \\
\hline Missing data & $0(0)$ & $0(0)$ & \\
\hline No & $167(73 \cdot 9)$ & $59(26 \cdot 1)$ & \\
\hline Yes & $10(38 \cdot 5)$ & $16(61 \cdot 5)$ & \\
\hline Bone marrow transplantation & & & $1+$ \\
\hline Missing data & $0(0)$ & $0(0)$ & \\
\hline No & $177(70 \cdot 2)$ & $75(29 \cdot 8)$ & \\
\hline Yes & 0 & 0 & \\
\hline Recent weight loss $>5 \mathrm{kgs}$ & & & $0 \cdot 082 *$ \\
\hline Missing data & $1(0 \cdot 6)$ & $2(2 \cdot 7)$ & \\
\hline No & $149(73)$ & $55(27)$ & \\
\hline Yes & $27(60)$ & $18(40)$ & \\
\hline Hemodialysis & & & $0 \cdot 321+$ \\
\hline Missing data & $0(0)$ & $0(0)$ & \\
\hline No & $173(69 \cdot 8)$ & $75(30 \cdot 2)$ & \\
\hline Yes & $4(100)$ & $0(0)$ & \\
\hline \multicolumn{4}{|l|}{ Clinical / Biological parameters } \\
\hline Intensive care unit management & & & $0 \cdot 029 *$ \\
\hline Missing data & $0(0)$ & $0(0)$ & \\
\hline No & $78(78)$ & $22(22)$ & \\
\hline Yes & $99(65 \cdot 1)$ & $53(34 \cdot 9)$ & \\
\hline Multiorgan failure & & & $<0.001 *$ \\
\hline Missing data & $0(0)$ & $0(0)$ & \\
\hline No & $158(77 \cdot 8)$ & $45(22 \cdot 2)$ & \\
\hline Yes & $19(38 \cdot 8)$ & $30(61 \cdot 2)$ & \\
\hline Aggravation of any pre existing organ dysfunction & & & $<0 \cdot 001^{*}$ \\
\hline Missing data & $0(0)$ & $0(0)$ & \\
\hline No & $150(77 \cdot 3)$ & $44(22 \cdot 7)$ & \\
\hline Yes & $27(46 \cdot 6)$ & $31(53 \cdot 4)$ & \\
\hline Systolic blood pressure $<80 \mathrm{mmHg}$ & & & $0 \cdot 499+$ \\
\hline Missing data & $2(1 \cdot 1)$ & $3(4)$ & \\
\hline No & $174(71)$ & $71(29)$ & \\
\hline Yes & $1(50)$ & $1(50)$ & \\
\hline
\end{tabular}


Diastolic blood pressure $<60 \mathrm{mmHg}$

Missing data

No

Heart rate $>120 /$ min

Missing data

No

Yes

Glasgow coma scale

Missing data

Min/Max

Med [Q1 ; Q3]

Mean (sd)

Diarrhoea

Missing data

No

Yes

Septic shock

Missing data

No

Yes

Flu-like symptoms

Missing data

No

Yes

Mechanical ventilation

Missing data

No

Yes

Hydrocephaly

Missing data

No

Yes

C-reactive protein level- $\mathrm{mg} / \mathrm{L}$

Missing data

Min/Max

Med [Q1; Q3]

Mean (sd)

Blood monocytes count $<200 / \mathrm{mm} 3$

Missing data

No

Yes

Blood polymorphonuclear cells count- cells $/ \mathrm{mm} 3$

Missing data

Min/Max

Med [Q1 ; Q3]

Mean (sd)

Blood lymphocytes count $<1500 / \mathrm{mm} 3$

Missing data

No

Yes

Clonal complex

Missing data

Other

CC1 24 or 6

Positive blood cultures for L. monocytogenes

Missing data

No

Yes

Encephalitis $^{++}$

Missing data

No

Yes

Number of neurological signs

Missing data
$2(1 \cdot 1)$

$158(71 \cdot 2)$

$17(68)$

7 (4)

$154(72 \cdot 3)$

$16(64)$

$5(2 \cdot 8)$

$3 / 15$

$14[11 ; 15]$

$12 \cdot 58(2 \cdot 96)$

$0(0)$

$149(69 \cdot 6)$

$28(73 \cdot 7)$

$0(0)$

$173(70)$

$4(80)$

$0(0)$

$122(66 \cdot 7)$

$55(79 \cdot 7)$

$0(0)$

$133(78 \cdot 7)$

$44(53)$

$0(0)$

$173(69 \cdot 8)$

4 (100)

$14(7 \cdot 9)$

$1 / 346$

$81[39 ; 166]$

$104 \cdot 52(81 \cdot 14)$

$15(8 \cdot 5)$

$152(73 \cdot 8)$

$10(41 \cdot 7)$

$2(1 \cdot 1)$

$540 / 34900$

$9764[6440 ; 13201]$

$10068 \cdot 7(5418 \cdot 9)$

$3(1 \cdot 7)$

$38(74 \cdot 5)$

$136(69 \cdot 4)$

$2(1 \cdot 1)$

$79(73 \cdot 1)$

$96(67 \cdot 6)$

$0(0)$

$82(87 \cdot 2)$

$95(60 \cdot 1)$

0 (0)

$32(91 \cdot 4)$

$145(66 \cdot 8)$

0 (0)
$3(4)$

$64(28 \cdot 8)$

$8(32)$

$0.741 *$

$0 \cdot 385^{*}$

$7(9 \cdot 3)$

$59(27 \cdot 7)$

$9(36)$

$0.001 \ddagger$

3 (4)

$3 / 15$

$12[9 ; 14]$

$11 \cdot 04(3 \cdot 42)$

$0 \cdot 614 *$

$0(0)$

$65(30 \cdot 4)$

$10(26 \cdot 3)$

$74(30)$

1 (20)

$0(0)$

$61(33 \cdot 3)$

$14(20 \cdot 3)$

$0(0)$

$36(21 \cdot 3)$

39 (47)

$0(0)$

$75(30 \cdot 2)$

$0(0)$

$5(6 \cdot 7)$

$6 / 433$

$89 \cdot 5[59 \cdot 25 ; 189 \cdot 5]$

$131 \cdot 69(105 \cdot 05)$

$7(9 \cdot 3)$

$54(26 \cdot 2)$

$14(58 \cdot 3)$

$2(2 \cdot 7)$

$20 / 43976$

$8700[5390 ; 12900]$

$9568(6879 \cdot 8)$

$2(2 \cdot 7)$

$13(25 \cdot 5)$

$60(30 \cdot 6)$

$0 \cdot 343^{*}$

$0(0)$

$29(26 \cdot 9)$

$46(32 \cdot 4)$

$0(0)$

$12(12 \cdot 8)$

$63(39 \cdot 9)$

$0(0)$

$3(8 \cdot 6)$

$72(33 \cdot 2)$

$0(0)$

$0 \cdot 043^{*}$

$<0.001 *$

$0 \cdot 321+$

$0.056+$

$0 \cdot 001 *$

$0.581+$

$0 \cdot 475^{*}$

$<0.001 *$

$0.003 *$

$0.285 \div$ 
$\operatorname{Min} / \operatorname{Max}$

Med [Q1; Q3]

Mean (sd)

Nucleated cells number in the cerebrospinal fluid

Missing data

Min/Max

Med [Q1; Q3]

Mean (sd)

Polymorphonuclear cells number in the cerebrospinal fluid

Missing data

Min/Max

Med [Q1; Q3]

Mean (sd)

Polymorphonuclear cells /all nucleated cells ratio

Missing data

Min/Max

Med [Q1 ; Q3]

Mean (sd)

Protein levels in the cerebrospinal fluid

Missing data

Min/Max

Med [Q1 ; Q3]

Mean (sd)

Cerebrospinal/blood glucose ratio

Missing data

Min/Max

Med [Q1 ; Q3]

Mean (sd)

Treatment

Time interval from diagnosis to treatment

Missing data

No treatment

$<0 \mathrm{~d}$

$[0 ; 1] \mathrm{d}$

$>1 \mathrm{~d}$

Time interval from admission to treatment

Missing data

No treatment

$[0 ; 1] \mathrm{d}$

$$
>1 \mathrm{~d}
$$

Time interval from first symptom to treatment

Missing data

No treatment

$<0 \mathrm{~d}$

$[0 ; 1] \mathrm{d}$

$$
>1 \mathrm{~d}
$$

Duration of bitherapy

Anti-listeria betalactamin $^{* *}+$ aminoglycoside

Missing data

$0 \mathrm{~d}$

$<=3 \mathrm{~d}$

$>3 \mathrm{~d}$

Duration of bitherapy

Cotrimoxazole + aminoglycoside

Missing data

$0 \mathrm{~d}$

$<=3 \mathrm{~d}$

$>3 \mathrm{~d}$

Duration of tritherapy ${ }^{*}$

Anti-listeria betalactam, cotrimoxazole and aminoglycoside Missing data

$0 \mathrm{~d}$

$<=3 \mathrm{~d}$

$$
>3 \mathrm{~d}
$$

Cotrimoxazole administration

Missing data

No
$8(4 \cdot 5)$

$0 / 12400$

$500[200 ; 1150]$

$1108 \cdot 43(1868 \cdot 68)$

$11(6 \cdot 2)$

$0 / 9520$

$265 \cdot 5[76 \cdot 25 ; 795]$

$775 \cdot 27(1423 \cdot 15)$

$12(6 \cdot 8)$

$0 / 100$

$64[38 ; 84 \cdot 1]$

$58 \cdot 14(29 \cdot 18)$

$8(4 \cdot 5)$

$0 \cdot 3 / 8 \cdot 9$

$1.9[1 \cdot 3 ; 2 \cdot 9]$

$2 \cdot 32(1 \cdot 5)$

$39(22)$

$0 \cdot 01 / 1 \cdot 4$

$0.33[0.23 ; 0.52]$

$0.39(0.23)$

$0.39(0.23)$

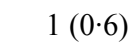

$0(0)$

$34(60 \cdot 7)$

$120(73 \cdot 2)$

$22(73 \cdot 3)$

$1(0 \cdot 6)$

$0(0)$

$142(70 \cdot 6)$

$34(69 \cdot 4)$

$$
2(1 \cdot 1)
$$

$0(0)$

$2(100)$

$68(64 \cdot 8)$

$105(77 \cdot 8)$

$0(0)$

$29(59 \cdot 2)$

$27(57 \cdot 4)$

$121(77 \cdot 6)$

$0(0)$

$163(70 \cdot 3)$

$10(71 \cdot 4)$

$4(66 \cdot 7)$

$0(0)$

$164(70 \cdot 4)$

$9(69 \cdot 2)$

$4(66 \cdot 7)$

$0(0)$

$144(68 \cdot 6)$
$0 / 8$

$2[1 \cdot 5 ; 3 \cdot 5]$

$2.55(1.56)$

9 (12)

$0 / 8484$

$355[128 \cdot 75 ; 984]$

$908 \cdot 83(1459 \cdot 48)$

$10(13 \cdot 3)$

$0 / 7395$

$181[56 ; 422]$

$658 \cdot 71(1244 \cdot 65)$

$11(14 \cdot 7)$

$0 / 100$

$67 \cdot 35[42 \cdot 62 ; 81 \cdot 25]$

$60 \cdot 24(27 \cdot 86)$

9 (12)

$0 \cdot 4 / 20$

$2 \cdot 6[1 \cdot 7 ; 3 \cdot 98]$

$3.47(3.46)$

$16(21 \cdot 3)$

$0 \cdot 02 / 0 \cdot 97$

$0.27[0 \cdot 16 ; 0 \cdot 45]$ $0 \cdot 34(0 \cdot 26)$

$0 \cdot 303 \S$

$0.615 \$$

$0 \cdot 011 \ddagger$

$0.036 \S$

$0 \cdot 121 \dagger$

$0(0)$

$1(100)$

$22(39 \cdot 3)$

$44(26 \cdot 8)$

$8(26 \cdot 7)$

$1(100)$

$59(29 \cdot 4)$

$15(30 \cdot 6)$

$7(9 \cdot 3)$

$1(100)$

$0(0)$

$37(35 \cdot 2)$

$30(22 \cdot 2)$

$0(0)$

$20(40 \cdot 8)$

$20(42 \cdot 6)$

$35(22 \cdot 4)$

$0 \cdot 025+$

0.005*

$0(0)$

$69(29 \cdot 7)$

$4(28 \cdot 6)$

$2(33 \cdot 3)$

0 (0)

$69(29 \cdot 6)$

$4(30 \cdot 8)$

$2(33 \cdot 3)$

$0(0)$

$66(31 \cdot 4)$ 
Yes

Aminoglycoside administration

Missing data

No

Yes

Anti-listeria betalactam administration ${ }^{* *}$

Missing data

No

Yes

Adjunctive dexamethasone prescription

Missing data

No

Yes
$9(21 \cdot 4)$

$0(0)$

$19(40 \cdot 4)$

$56(27 \cdot 3)$

$0 \cdot 053+$

$5(62 \cdot 5)$

$70(28 \cdot 7)$

$1(1 \cdot 3)$

$59(27 \cdot 3)$

$15(46 \cdot 9)$

\section{D· Univariable analysis of persisting neurological impairment in neurolisteriosis cases}

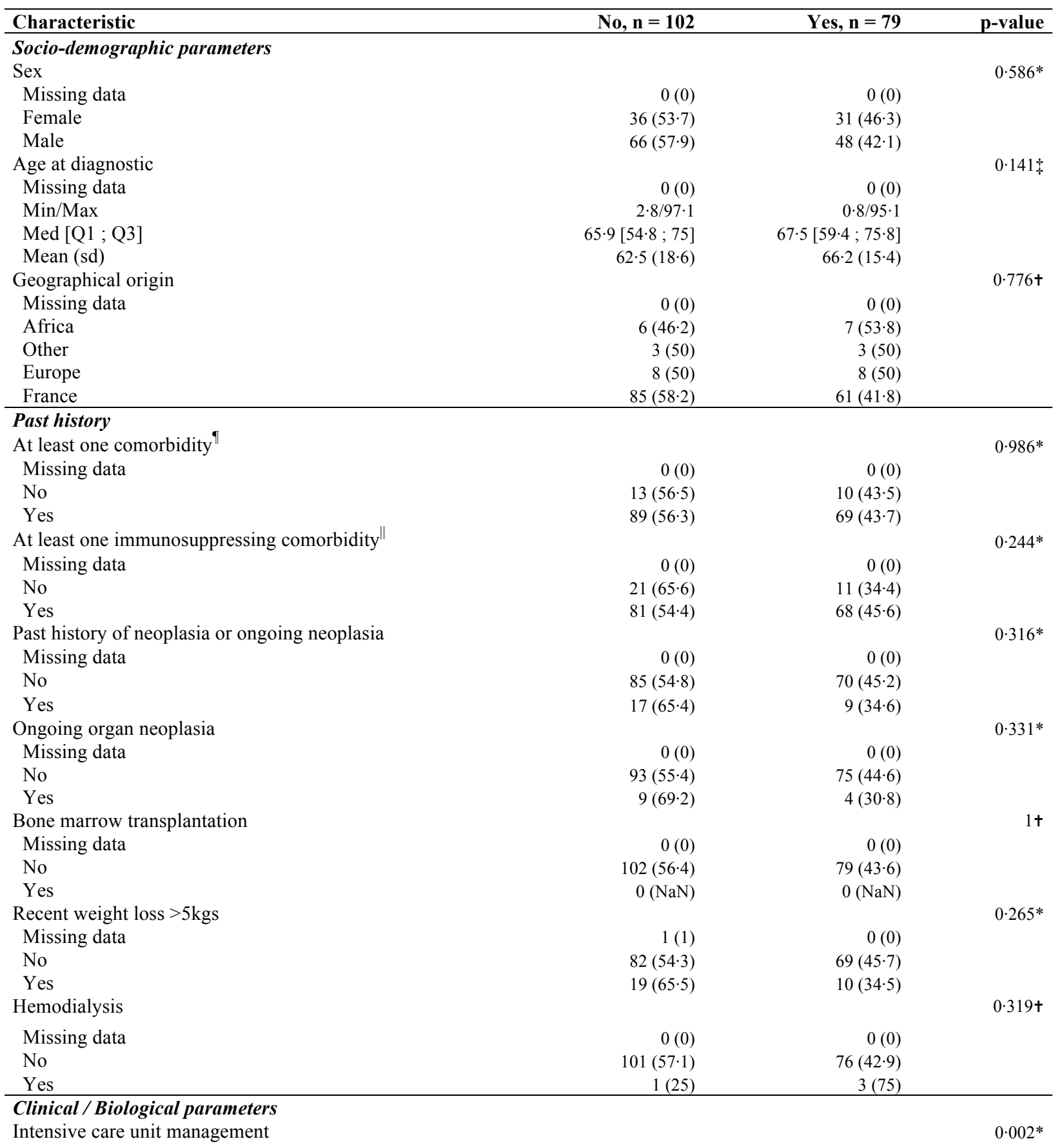


Missing data

No

Yes

Multiorgan failure

Missing data

No

Yes

Aggravation of any pre existing organ dysfunction

Missing data

No

Yes

Systolic blood pressure $<80 \mathrm{mmHg}$

Missing data

No

Yes

Diastolic blood pressure $<60 \mathrm{mmHg}$

Missing data

No

Yes

Heart rate $>120 / \mathrm{min}$

Missing data

No

Yes

Glasgow coma scale

Missing data

Min/Max

Med [Q1 ; Q3]

Mean (sd)

Diarrhoea

Missing data

No

Yes

Septic shock

Missing data

No

Yes

Flu-like symptoms

Missing data

No

Yes

Mechanical ventilation

Missing data

No

Yes

Hydrocephaly

Missing data

No

Yes

C-reactive protein level- $\mathrm{mg} / \mathrm{L}$

Missing data

Min/Max

Med [Q1; Q3]

Mean (sd)

Blood monocytes count $<200 / \mathrm{mm} 3$

Missing data

No

Yes

Blood polymorphonuclear cells count - cells $/ \mathrm{mm} 3$

Missing data

Min/Max

Med [Q1; Q3]

Mean (sd)

Blood lymphocytes count $<1500 / \mathrm{mm} 3$

Missing data

\begin{tabular}{|c|c|c|}
\hline $0(0)$ & $0(0)$ & \\
\hline $55(69 \cdot 6)$ & $24(30 \cdot 4)$ & \\
\hline $47(46 \cdot 1)$ & $55(53 \cdot 9)$ & \\
\hline & & $0 \cdot 012 *$ \\
\hline $0(0)$ & $0(0)$ & \\
\hline $96(59 \cdot 6)$ & $65(40 \cdot 4)$ & \\
\hline $6(30)$ & $14(70)$ & \\
\hline & & $0 \cdot 461 *$ \\
\hline $0(0)$ & $0(0)$ & \\
\hline $88(57 \cdot 5)$ & $65(42 \cdot 5)$ & \\
\hline $14(50)$ & $14(50)$ & \\
\hline $3(2 \cdot 9)$ & $0(0)$ & \\
\hline $98(55 \cdot 4)$ & $79(44 \cdot 6)$ & \\
\hline $1(100)$ & $0(0)$ & \\
\hline & & $0 \cdot 268 *$ \\
\hline $3(2 \cdot 9)$ & $0(0)$ & \\
\hline $88(54 \cdot 3)$ & $74(45 \cdot 7)$ & \\
\hline $11(68 \cdot 8)$ & $5(31 \cdot 2)$ & \\
\hline & & $0 \cdot 284 *$ \\
\hline $5(4 \cdot 9)$ & $4(5 \cdot 1)$ & \\
\hline $90(57 \cdot 7)$ & $66(42 \cdot 3)$ & \\
\hline $7(43 \cdot 8)$ & $9(56 \cdot 2)$ & \\
\hline & & $0.043 \ddagger$ \\
\hline $4(3 \cdot 9)$ & $1(1 \cdot 3)$ & \\
\hline $3 / 15$ & $3 / 15$ & \\
\hline $14[12 \cdot 25 ; 15]$ & $13[11 ; 14]$ & \\
\hline $13 \cdot 02(2 \cdot 66)$ & $12 \cdot 12(3 \cdot 12)$ & \\
\hline & & $0.927 *$ \\
\hline $0(0)$ & $0(0)$ & \\
\hline $86(56 \cdot 2)$ & $67(43 \cdot 8)$ & \\
\hline $16(57 \cdot 1)$ & $12(42 \cdot 9)$ & \\
\hline & & $0 \cdot 319 t$ \\
\hline $0(0)$ & $0(0)$ & \\
\hline $101(57 \cdot 1)$ & $76(42 \cdot 9)$ & \\
\hline $1(25)$ & $3(75)$ & \\
\hline & & $0 \cdot 314^{*}$ \\
\hline $0(0)$ & $0(0)$ & \\
\hline $73(58 \cdot 9)$ & $51(41 \cdot 1)$ & \\
\hline $29(50 \cdot 9)$ & $28(49 \cdot 1)$ & \\
\hline & & $0 \cdot 001^{*}$ \\
\hline $0(0)$ & $0(0)$ & \\
\hline $86(63 \cdot 2)$ & $50(36 \cdot 8)$ & \\
\hline $16(35 \cdot 6)$ & $29(64 \cdot 4)$ & \\
\hline & & $0.035 t$ \\
\hline $0(0)$ & $0(0)$ & \\
\hline $102(57 \cdot 6)$ & $75(42 \cdot 4)$ & \\
\hline $0(0)$ & $4(100)$ & \\
\hline & & $0 \cdot 727 \ddagger$ \\
\hline $8(7 \cdot 8)$ & $6(7 \cdot 6)$ & \\
\hline $3 / 433$ & $1 / 323$ & \\
\hline $82.5[44 \cdot 5 ; 168]$ & $93[33 ; 165]$ & \\
\hline $109.93(88.28)$ & $105 \cdot 38(79 \cdot 2)$ & \\
\hline & & $0 \cdot 298^{*}$ \\
\hline $9(8 \cdot 8)$ & $6(7 \cdot 6)$ & \\
\hline $88(57 \cdot 1)$ & $66(42 \cdot 9)$ & \\
\hline $5(41 \cdot 7)$ & $7(58 \cdot 3)$ & \\
\hline $1(1)$ & $1(1 \cdot 3)$ & $0.896 \ddagger$ \\
\hline $450 / 34900$ & $1031 / 29000$ & \\
\hline $976[6345 ; 13370]$ & $9610[6522 \cdot 5 ; 12971 \cdot 5]$ & \\
\hline $9984 \cdot 3(5692 \cdot 3)$ & $10089 \cdot 9(5112 \cdot 3)$ & \\
\hline $2(2)$ & $1(1 \cdot 3)$ & $0 \cdot 410^{*}$ \\
\hline
\end{tabular}


No

Yes

Clonal complex

Missing data

Other

CC1 24 or 6

Positive blood cultures for L. monocytogenes

Missing data

No

Yes

Encephalitis $^{\dagger+}$

Missing data

No

Yes

Number of neurological signs

Missing data

Min/Max

Med [Q1 ; Q3]

Mean (sd)

Nucleated cells number in the cerebrospinal fluid

Missing data

Min/Max

Med [Q1 ; Q3]

Mean (sd)

Polymorphonuclear cells number in the cerebrospinal fluid

Missing data

Min/Max

Med [Q1 ; Q3]

Mean (sd)

Polymorphonuclear cells /all nucleated cells ratio

Missing data

Min/Max

Med [Q1; Q3]

Mean (sd)

Protein levels in the cerebrospinal fluid

Missing data

Min/Max

Med [Q1 ; Q3]

Mean (sd)

Cerebrospinal/blood glucose ratio

Missing data

Min/Max

Med [Q1; Q3]

Mean (sd)

Treatment

Time interval from diagnosis to treatment

Missing data

$<0 \mathrm{~d}$

$[0 ; 1] \mathrm{d}$

$>1 \mathrm{~d}$

Time interval from admission to treatment

Missing data

$[0 ; 1] \mathrm{d}$

$>1 \mathrm{~d}$

Time interval from first symptom to treatment

Missing data

$<0 \mathrm{~d}$

$[0 ; 1] \mathrm{d}$

$>1 \mathrm{~d}$

Duration of bitherapy

Anti-listeria betalactamin + aminoglycoside**

Missing data

$0 \mathrm{~d}$

$<=3 \mathrm{~d}$

$>3 \mathrm{~d}$

Duration of bitherapy

\begin{tabular}{|c|c|c|}
\hline $23(62 \cdot 2)$ & $14(37 \cdot 8)$ & \\
\hline $77(54 \cdot 6)$ & $64(45 \cdot 4)$ & \\
\hline & & $0 \cdot 719 *$ \\
\hline $2(2)$ & $0(0)$ & \\
\hline $47(57 \cdot 3)$ & $35(42 \cdot 7)$ & \\
\hline $53(54 \cdot 6)$ & $44(45 \cdot 4)$ & \\
\hline & & $0.59^{*}$ \\
\hline $0(0)$ & $0(0)$ & \\
\hline $48(58 \cdot 5)$ & $34(41 \cdot 5)$ & \\
\hline $54(54 \cdot 5)$ & $45(45 \cdot 5)$ & \\
\hline & & $<0.001 *$ \\
\hline $0(0)$ & $0(0)$ & \\
\hline $31(96 \cdot 9)$ & $1(3 \cdot 1)$ & \\
\hline $71(47 \cdot 7)$ & $78(52 \cdot 3)$ & \\
\hline & & $<0.001 \ddagger$ \\
\hline $0(0)$ & $0(0)$ & \\
\hline $0 / 6$ & $1 / 8$ & \\
\hline $2[1 ; 2]$ & $3[2 ; 3]$ & \\
\hline $1.9(1 \cdot 21)$ & $2.96(1.57)$ & \\
\hline & & $0.567 \ddagger$ \\
\hline $6(5 \cdot 9)$ & $4(5 \cdot 1)$ & \\
\hline $0 / 12400$ & $0 / 11825$ & \\
\hline $490[200 ; 1108 \cdot 5]$ & $456[162 ; 1125]$ & \\
\hline $1158.9(2017 \cdot 01)$ & $998 \cdot 13(1646 \cdot 38)$ & \\
\hline & & $0.465 \S$ \\
\hline $7(6 \cdot 9)$ & $5(6 \cdot 3)$ & \\
\hline $0 / 9520$ & $0 / 8375$ & \\
\hline $274[89 \cdot 5 ; 702 \cdot 5]$ & $212[51 ; 795]$ & \\
\hline $809 \cdot 17(1520 \cdot 32)$ & $682 \cdot 26(1264 \cdot 59)$ & \\
\hline & & $0 \cdot 246 \ddagger$ \\
\hline $8(7 \cdot 8)$ & $5(6 \cdot 3)$ & \\
\hline $0 / 100$ & $0 / 95 \cdot 1$ & \\
\hline $70[40 \cdot 33 ; 85 \cdot 07]$ & $58.95[34.47 ; 80]$ & \\
\hline $60 \cdot 43(29 \cdot 31)$ & $55 \cdot 24(28 \cdot 12)$ & \\
\hline & & $0.093 \ddagger$ \\
\hline $6(5 \cdot 9)$ & $4(5 \cdot 1)$ & \\
\hline $0 \cdot 3 / 6$ & $0 \cdot 4 / 8 \cdot 9$ & \\
\hline $1 \cdot 8[1 \cdot 2 ; 2 \cdot 8]$ & $2 \cdot 2[1 \cdot 4 ; 3 \cdot 2]$ & \\
\hline $2 \cdot 12(1 \cdot 28)$ & $2.52(1.71)$ & \\
\hline & & $0.935 \S$ \\
\hline $25(24 \cdot 5)$ & $16(20 \cdot 3)$ & \\
\hline $0 \cdot 05 / 1 \cdot 17$ & $0 \cdot 01 / 1 \cdot 4$ & \\
\hline $0 \cdot 33[0 \cdot 24 ; 0.51]$ & $0 \cdot 36[0 \cdot 22 ; 0.55]$ & \\
\hline $0 \cdot 38(0 \cdot 21)$ & $0 \cdot 4(0 \cdot 27)$ & \\
\hline & & $0.571^{\dagger}$ \\
\hline $1(1)$ & $0(0)$ & \\
\hline $17(50)$ & $17(50)$ & \\
\hline $68(56 \cdot 2)$ & $53(43 \cdot 8)$ & \\
\hline $16(64)$ & $9(36)$ & \\
\hline & & $0.579^{\dagger}$ \\
\hline $1(1)$ & $0(0)$ & \\
\hline $82(57 \cdot 3)$ & $61(42 \cdot 7)$ & \\
\hline $19(51 \cdot 4)$ & $18(48 \cdot 6)$ & \\
\hline & & $0.939^{\dagger}$ \\
\hline $1(1)$ & $1(1 \cdot 3)$ & \\
\hline $1(50)$ & $1(50)$ & \\
\hline $38(55 \cdot 1)$ & $31(44 \cdot 9)$ & \\
\hline $62(57 \cdot 4)$ & $46(42 \cdot 6)$ & \\
\hline & & $0.678^{*}$ \\
\hline $0(0)$ & $0(0)$ & \\
\hline $19(61 \cdot 3)$ & $12(38 \cdot 7)$ & \\
\hline $17(60 \cdot 7)$ & $11(39 \cdot 3)$ & \\
\hline $66(54 \cdot 1)$ & $56(45 \cdot 9)$ & \\
\hline & & $0.139^{\dagger}$ \\
\hline
\end{tabular}


Cotrimoxazole + aminoglycoside

Missing data

$0 \mathrm{~d}$

$$
\begin{aligned}
& <=3 \mathrm{~d} \\
& >3 \mathrm{~d}
\end{aligned}
$$

Duration of tritherapy**

Anti-listeria betalactam, cotrimoxazole and aminoglycoside

Missing data

$0 \mathrm{~d}$

$<=3 \mathrm{~d}$

$>3 \mathrm{~d}$

Cotrimoxazole administration

Missing data

Aminoglycoside administration

No

Anti-listeria betalactam administration**

Missing data

Yes

Adjunctive dexamethasone prescription Missing data

\section{Table S5}

\begin{tabular}{lcc}
\hline \multicolumn{1}{c}{ Factors } & Odds ratio $(\mathbf{9 5 \%} \mathbf{C I})^{*}$ & p-value \\
\hline $\begin{array}{l}\text { Maternal urinary tract symptoms } \\
\text { Time interval from diagnosis to treatment }\end{array}$ & $4 \cdot 73(0 \cdot 16-142 \cdot 53)$ & $0 \cdot 371$ \\
(Reference $=$ no treatment) & & \\
$<0$ day & $9 \cdot 73(0 \cdot 38-246 \cdot 18)$ & $0 \cdot 168$ \\
{$[0 ; 1]$ day } & $1 \cdot 03(0 \cdot 04-23 \cdot 75)$ & $0 \cdot 984$ \\
$>1$ day & $2 \cdot 99(0 \cdot 07-125 \cdot 84)$ & $0 \cdot 566$ \\
Term at maternal diagnosis of listeriosis & & \\
$(\text { Reference }=[0,25 \mathrm{WG}))^{+}$ & & $0 \cdot 610$ \\
{$[25,28 \mathrm{WG})$} & $0 \cdot 58(007-481)$ & $<0 \cdot 001$ \\
{$[28,41 \mathrm{WG}]$} & $0 \cdot 01(0-0 \cdot 05)$ & \\
\hline
\end{tabular}




\section{Titles and legends for supplemental tables}

\section{Table S1 Characteristics of the patients included and not included in the study}

* Mortality data were those collected at 3-month for included patients and at the moment of screening for nonincluded patients.

${ }^{\dagger}$ Mann-Whitney test.

* Age was evaluated in 22 patients.

$\S$ Chi-squared test.

Table S2 Obstetrical and neonatal outcome of maternal-neonatal cases according to the term of pregnancy at diagnosis

* The study involved the 107 cases of maternal-neonatal infections, including 4 mothers with twin pregnancies, and the 82 neonates born alive. Values inside brackets denote interquartile range [25\%; 75\%].

${ }^{\dagger}$ Abnormal delivery at term was defined as delivery with fever and/or meconium fluid and/or abnormal fetal heart rate.

* The neonates group included one pair of twins.

$\S$ Neonates were considered healthy if they had normal examination at birth, C-reactive protein level $<5 \mathrm{mg} / \mathrm{L}$, nucleated cells count in the cerebrospinal fluid $\leq 30 / \mathrm{mm}^{3}$ (in case a lumbar puncture was performed) and all bacteriological samples negative.

Table S3 Detailed associated comorbidities of the study population

* Patients were: 427 cases with bacteremia, 252 cases with neurolisteriosis, 107 cases with maternal-neonatal infection, Plus-minus values are means $\pm \mathrm{SD}$.

${ }^{\dagger}$ Chronic liver diseases were: alcoholic liver disease $(n=64)$, viral hepatitis $C(n=10)$, viral hepatitis $B(n=9)$, and other liver disease $(n=31)$.

*Foreign materials or implants were: bone/joint prosthetic devices $(n=54)$, cardiac valve/prosthetic arterial tubes $(n=61)$, pacemakers $(n=37)$, central venous catheters $(n=53)$, other types of material $(n=37)$ Patients could report more than one foreign material.

${ }^{\S}$ Solid organ transplantation involved the kidney $(n=15)$ and the heart $(n=5)$.

" Patients with HIV infection had a mean $\mathrm{CD}^{+}$cells count of $239 / \mathrm{mm}^{3}( \pm 214), 8$ reported antiretroviral treatment, and 1 reported cotrimoxazole prophylaxis.

"Inflammatory bowel diseases include Crohn disease and hemorrhagic recto-colitis.

** Inflammatory rheumatic disorders included: rheumatoid arthritis, ankylosing spondylitis, polymyalgia rheumatic, psoriatic arthritis and other non-specified inflammatory rheumatic disorders.

Table S4 Univariable analyses of fetal loss in maternal cases, of 3-month death in bacteremia + neurolisteriosis cases, of 3-month death in neurolisteriosis cases, and of of persisting neurological impairment in neurolisteriosis cases.

* Chi-squared test.

${ }^{\dagger}$ Fisher-exact test.

*Student's t-test.

${ }^{\S}$ Mann-Whitney test.

" Comorbidities included: chronic respiratory disease, hypertension, chronic heart disease, cardiopathy, thrombophilia, hemoglobinopathy, seizures, psychiatric disorder, atopy, renal insufficiency, chronic liver disease, foreign material or implant, recent major weight loss, daily alcohol uptake $>3$ glasses / day, cirrhosis, diabetes mellitus, end stage renal disease, solid organ cancer, hematological malignancy, hematopoietic stem cell transplantation, solid organ transplantation, asplenia, pre-existing neutropenia (neutrophils count $<500 / \mathrm{mm}^{3}$ ), pre-existing lymphopenia (lymphocytes count $<1000 / \mathrm{mm}^{3}$ ), HIV infection, inflammatory bowel diseases (Crohn disease and hemorrhagic recto-colitis), inflammatory rheumatic disorders (rheumatoid arthritis, 
ankylosing spondylitis, polymyalgia rheumatic, psoriatic arthritis and other non-specified inflammatory rheumatic disorders), obesity, congenital immune deficiency, other auto-immune diseases, other comorbidity.

" Immunosuppressive comorbidities included: daily alcohol uptake $>3$ drinks/day, cirrhosis, diabetes mellitus, end-stage renal disease, solid organ cancer, hematological malignancy, hematopoietic stem cell transplantation, solid organ transplantation, asplenia, preexisting neutropenia, preexisting lymphopenia, HIV infection, inflammatory bowel diseases, inflammatory rheumatic disorders, other auto-immune diseases, congenital immune deficiency, age $>70$ years, prescription of corticosteroids or other immunosuppressive therapies in the last 5 years.

** Betalactams lacking activity towards Listeria $s p$ were excluded: oxacillin and cephalosporins.

${ }^{+\dagger}$ Encephalitis was defined by the presence of at least one of the following symptoms, with no alternative cause than listeriosis identified: altered consciousness (score on Glasgow Coma Scale <15), seizures, new onset of neurological symptoms and abnormality on electroencephalography consistent with encephalitis ${ }^{1}$

Table S5 Multivariable logistic regression analysis of factors associated with fetal loss for mothers with maternal infections (30 fetal loss out of 107 mothers).

* CI denotes confidence interval. Adjusted odds ratio were odds ratio calculated from the multivariable model after imputation of missing data.

${ }^{\dagger}$ WG denotes weeks of gestation.

\section{Titles and legends for supplemental figures}

Figure S1 A Multilocus sequence typing of 812 isolates from patients with listeriosis B Distribution of hypervirulent, hypovirulent and intermediate virulence clones for each form of infection Tests are Chi-squared tests unless specified otherwise.

* All available isolates were analyzed.

${ }^{\dagger}$ Fisher-exact test.

Figure S2 Biochemical and cellular features of the cerebrospinal fluid in the 235 patients with neurolisteriosis who had lumbar puncture performed. CSF denotes cerebrospinal fluid PMN denotes polymorphonuclear cells.

Figure S3 Inhibition zone diameters distribution of Listeria monocytogenes isolates with amoxicillin, cotrimoxazole and gentamicin.

Figure S4 Hierarchical clustering analysis of the patients with bacteremia and neurolisteriosis.

A Cluster dendrogram B Inertia gain C Factorial map resulting from the multiple correspondence analyses Each color denotes a specific cluster (cluster 1 in green, cluster 2 in yellow and cluster 3 in blue); triangles denote neurolisteriosis cases and crosses denote septicemia cases D Main characteristics of the clusters * This variable was not used for the classification. 


\section{References}

1 Venkatesan A, Tunkel AR, Bloch KC, Lauring AS, Sejvar J, Bitnun A, et al Case definitions, diagnostic algorithms, and priorities in encephalitis: consensus statement of the international encephalitis consortium Clin Infect Dis 2013; 57(8): 1114-28.

2 Gavazzi G, Krause KH. Ageing and infection. Lancet Infect Dis 2002; 2(11): 659-66.

3 Bone R, Balk R, Cerra F, Dellinger R, Fein A, Knaus W, et al. Definitions for sepsis and organ failure and guidelines for the use of innovative therapies in sepsis. The ACCP/SCCM Consensus Conference Committee. American College of Chest Physicians/Society of Critical Care Medicine. Chest 1992;101(6):1644-55.

4 von Elm E, Altman DG, Egger M, Pocock SJ, Gotzsche PC, Vandenbroucke JP The Strengthening the Reporting of Observational Studies in Epidemiology (STROBE) statement: guidelines for reporting observational studies Lancet 2007; 370(9596): 1453-7.

5 Cohen, Jacob (1988) Statistical Power Analysis for the Behavioral Sciences Routledge ISBN 1-134-74270-3.

6 Rubin DB, Schenker N Multiple imputation in health-care databases: An overview and some application Stat Med 1991;10:585-598.

7 Francois Husson, Julie Josse, Sebastien Le and Jeremy Mazet (2016) FactoMineR: Multivariate Exploratory Data Analysis and Data Mining R package version 132 http://CRANR-projectorg/package=FactoMineR.

8 Julie Josse, Francois Husson (2016) missMDA: A Package for Handling Missing Values in Multivariate Data Analysis Journal of Statistical Software, 70(1), 1-31 doi:1018637/jssv070i01.

9 R Core Team (2015) R: A language and environment for statistical computing R Foundation for Statistical Computing, Vienna, Austria URL https://wwwR-projectorg/. 


\section{A. Multi Locus Sequence Typing results of the 812 Listeria monocytogenes isolates *}

\begin{tabular}{|c|c|c|c|c|c|}
\hline \multirow[t]{2}{*}{ Clonal complexes (CC) - no. (\%) } & \multirow{2}{*}{$\begin{array}{c}\text { Maternal- } \\
\text { neonatal } \\
\text { isolates } \\
\mathrm{N}=106\end{array}$} & \multirow{2}{*}{$\begin{array}{c}\text { Bacteremia } \\
\text { isolates } \\
\quad \mathrm{N}=424\end{array}$} & \multirow{2}{*}{$\begin{array}{c}\begin{array}{c}\text { Neurolisteriosis } \\
\text { isolates }\end{array} \\
\mathrm{N}=\mathbf{2 5 0}\end{array}$} & \multicolumn{2}{|c|}{ p-value } \\
\hline & & & & $\begin{array}{c}\text { Neurolisteriosis } \\
\text { vs. bacteremia } \\
\text { cases }\end{array}$ & $\begin{array}{c}\text { Maternal vs. } \\
\text { non-maternal } \\
\text { cases }\end{array}$ \\
\hline Hypervirulent clones & $70(66)$ & $168(40)$ & $142(57)$ & $<0.001$ & $<0.001$ \\
\hline $\mathrm{CC} 1$ & $22(21)$ & $59(14)$ & $59(24)$ & 0.001 & 0.565 \\
\hline $\mathrm{CC} 4$ & $22(21)$ & $25(6)$ & $35(14)$ & $<0.001$ & $<0.001$ \\
\hline $\mathrm{CC} 2$ & $8(8)$ & $35(8)$ & $16(6)$ & 0.379 & 0.994 \\
\hline Hypovirulent clones & $0(0)$ & $34(8)$ & $12(5)$ & $0 \cdot 109$ & 0.006 \\
\hline CC9 & $0(0)$ & $19(4)$ & $4(2)$ & 0.047 & $0.060^{\dagger}$ \\
\hline $\mathrm{CC} 8$ & $1(1)$ & $25(6)$ & $12(5)$ & 0.546 & 0.043 \\
\hline $\mathrm{CC} 3$ & $5(5)$ & $7(2)$ & $5(2)$ & $0 \cdot 768^{* *}$ & $0.068^{+}$ \\
\hline $\mathrm{CC} 37$ & $0(0)$ & $18(4)$ & $3(1)$ & 0.028 & 0.098 \\
\hline CC101 + CC90 & $3(3)$ & $14(3)$ & $5(2)$ & $0 \cdot 324$ & $1^{\dagger}$ \\
\hline $\mathrm{CC} 7$ & $2(2)$ & $12(3)$ & $8(3)$ & 0.785 & $0.756^{+}$ \\
\hline CC155 & $2(2)$ & $11(3)$ & $4(2)$ & 0.398 & $1^{+}$ \\
\hline CC77 & $1(1)$ & $11(3)$ & $5(2)$ & 0.624 & $0.493^{+}$ \\
\hline Other & $12(11)$ & $107(25)$ & $44(18)$ & 0.022 & 0.009 \\
\hline
\end{tabular}

B.

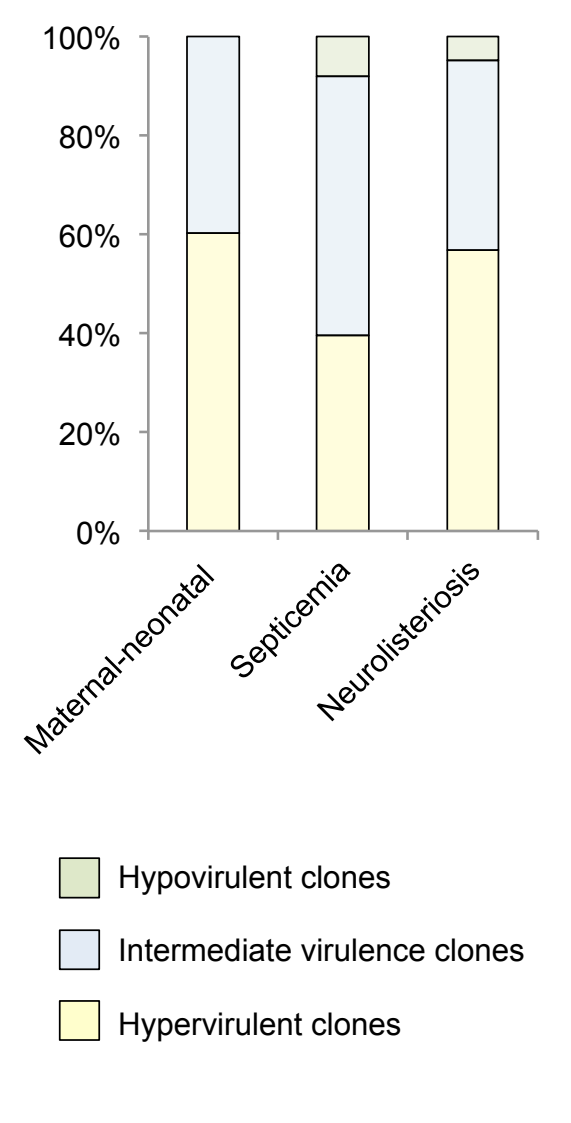




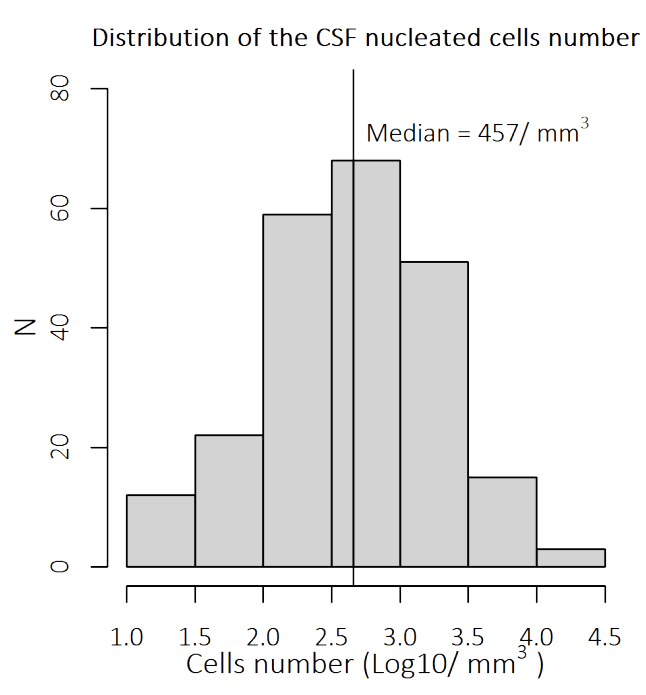

Distribution of the PMN : all nucleated cells ratio

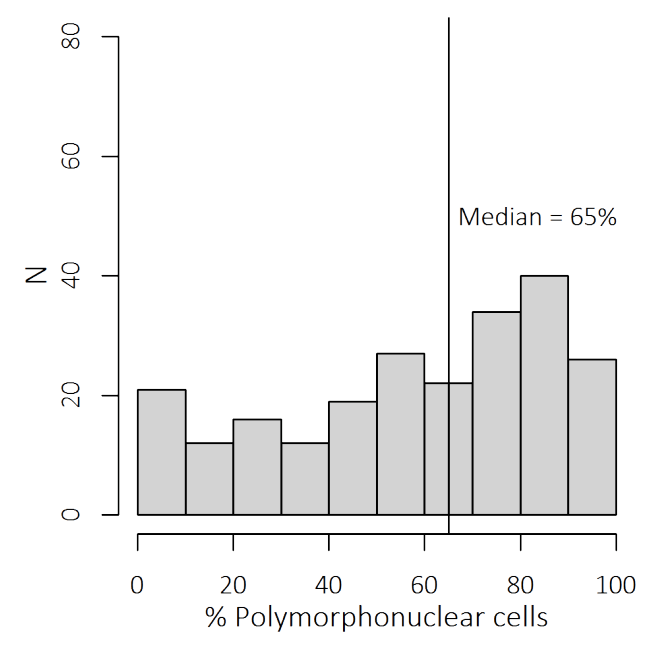

Distribution of protein levels in the CSF

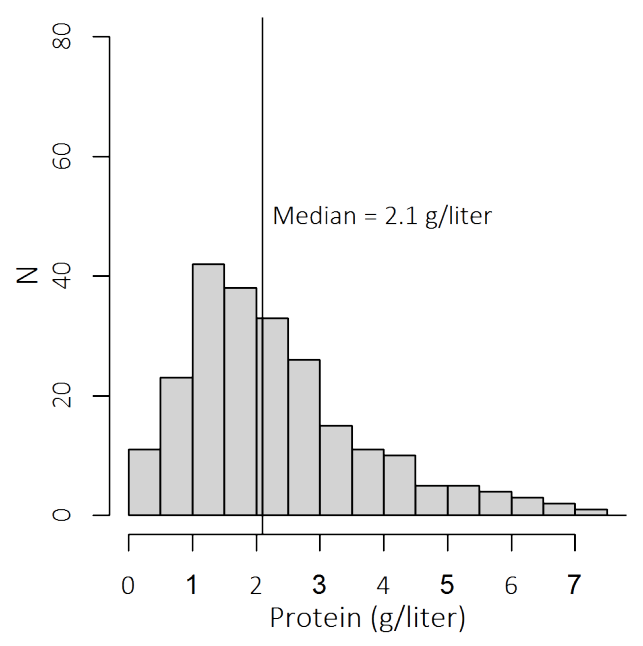

Distribution of the CSF : blood glucose ratio

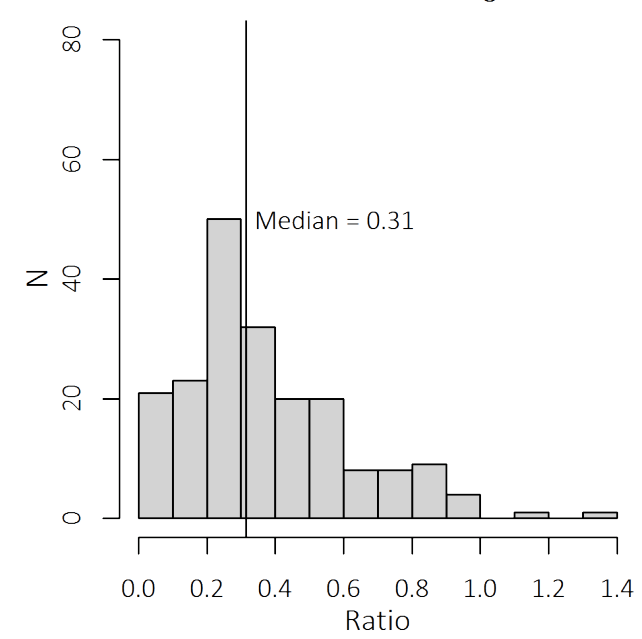

\section{Figure S2}


A.

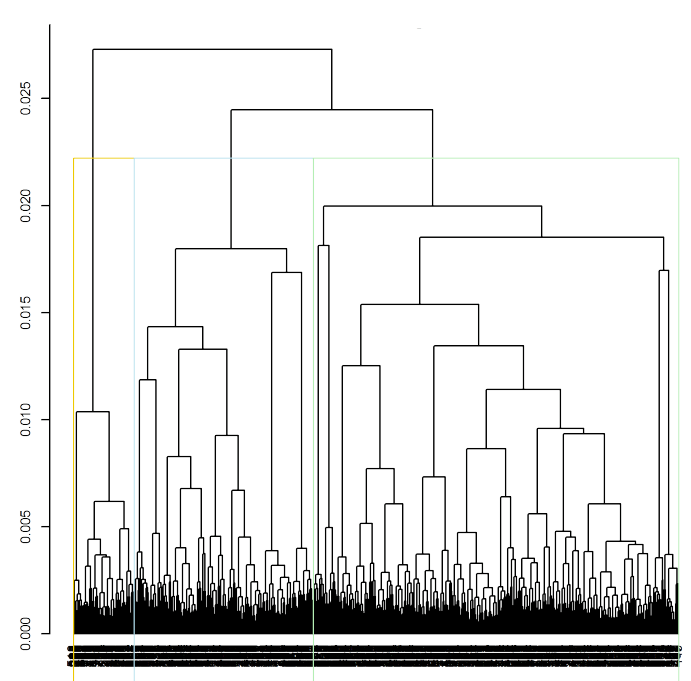

D. Main characteristics of clusters

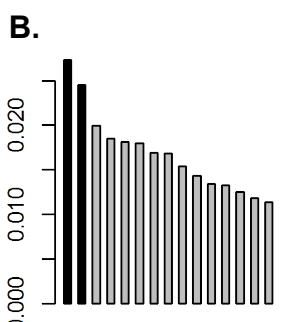

Inertia gain

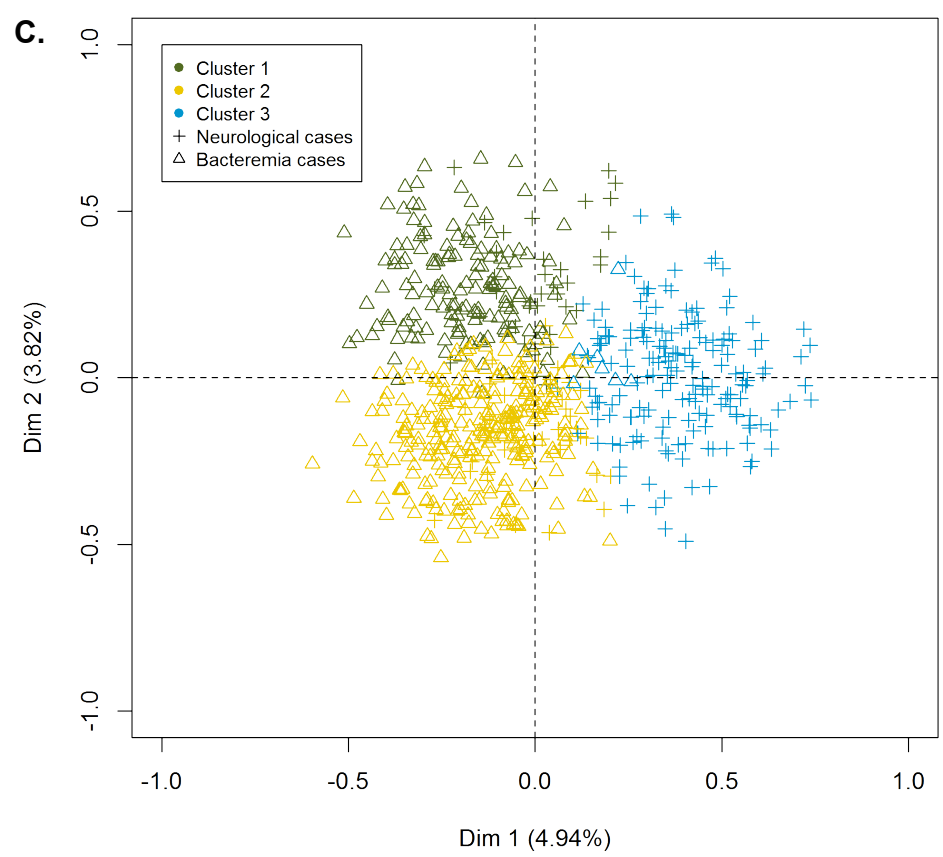

\begin{tabular}{|c|c|c|c|}
\hline & $\begin{array}{c}\text { Cluster } 1-\text { no. }(\%) \\
N=183\end{array}$ & $\begin{array}{c}\text { Cluster } 2-\text { no. }(\%) \\
N=314\end{array}$ & $\begin{array}{c}\text { Cluster } 3-\text { no. }(\%) \\
\mathrm{N}=182\end{array}$ \\
\hline \multicolumn{4}{|l|}{ Form of infection* } \\
\hline Bacteremia & $146 / 183(80 \%)$ & $274 / 314(87 \%)$ & $7 / 182(4 \%)$ \\
\hline Neurolisteriosis & $37 / 183(20 \%)$ & $40 / 314(13 \%)$ & $175 / 182(96 \%)$ \\
\hline Immunosuppressants & $144 / 183(79 \%)$ & $26 / 314(8 \%)$ & $38 / 182(21 \%)$ \\
\hline Mean number of comorbidities* & $3 \cdot 6 \pm 1 \cdot 9$ & $3 \cdot 7 \pm 2 \cdot 0$ & $2 \cdot 6 \pm 2 \cdot 0$ \\
\hline Mean number of immunosuppressive comorbidities* & $3 \cdot 9 \pm 1 \cdot 4$ & $2 \cdot 1 \pm 1 \cdot 3$ & $1 \cdot 9 \pm 1 \cdot 5$ \\
\hline Mean age in years & $68 \pm 12$ & $76 \pm 13$ & $64 \pm 18$ \\
\hline Percentage of patients with age $\geq 80$ years & $0 / 183(0 \%)$ & $56 / 314(18 \%)$ & $10 / 182(5 \%)$ \\
\hline Positive blood cultures & $177 / 183(97 \%)$ & $312 / 314(99 \%)$ & $96 / 182(53 \%)$ \\
\hline \multicolumn{4}{|l|}{ Strains } \\
\hline Hypervirulent & $59 / 182(32 \%)$ & $137 / 312(44 \%)$ & $114 / 181(63 \%)$ \\
\hline Intermediate & $99 / 182(54 \%)$ & $157 / 312(50 \%)$ & $62 / 181(34 \%)$ \\
\hline Hypovirulent & $24 / 182(13 \%)$ & $18 / 312(6 \%)$ & $4 / 181(2 \%)$ \\
\hline
\end{tabular}

Figure S4 